SLAC-R-1026

\title{
A Measurement of Neutral B \\ Meson Mixing Using Dilepton \\ Events with the BABAR Detector \\ by
}

Naveen Jeevaka Wickramasinghe Gunawardane

December 2000 


\section{A measurement of neutral $B$ meson mixing using dilepton events with the BABAR detector}

Naveen Jeevaka Wickramasinghe Gunawardane

Imperial College, London

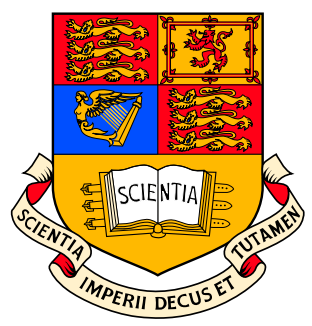

A thesis submitted for the degree of Doctor of Philosophy of the University of London and the Diploma of Imperial College

December, 2000 


\title{
A measurement of neutral $B$ meson mixing using dilepton events with the BABAR detector
}

\author{
Naveen Gunawardane \\ Blackett Laboratory \\ Imperial College of Science, Technology and Medicine \\ Prince Consort Road \\ London SW7 2BW
}

December, 2000

\begin{abstract}
This thesis reports on a measurement of the neutral B meson mixing parameter, $\Delta m_{d}$, at the BABAR experiment and the work carried out on the electromagnetic calorimeter (EMC) data acquisition (DAQ) system and simulation software.

The BABAR experiment, built at the Stanford Linear Accelerator Centre, uses the PEP-II asymmetric storage ring to make precise measurements in the B meson system. Due to the high beam crossing rates at PEP-II, the DAQ system employed by BABAR plays a very crucial role in the physics potential of the experiment.

The inclusion of machine backgrounds noise is an important consideration within the simulation environment. The BABAR event mixing software written for this purpose have the functionality to mix both simulated and real detector backgrounds. Due to the high energy resolution expected from the EMC, a matched digital filter is used. The performance of the filter algorithms could be improved upon by means of a polynomial fit. Application of the fit resulted in a $4-40 \%$ improvement in the energy resolution and a $90 \%$ improvement in the timing resolution.

A dilepton approach was used in the measurement of $\Delta m_{d}$ where the flavour of the $\mathrm{B}$ was tagged using the charge of the lepton. An unbinned maximum likelihood fitting procedure with an event-by-event error weighted resolution function was used in the extraction of $\Delta m_{d}$. With the $7.72 \mathrm{fb}^{-1}$ of $\Upsilon(4 \mathrm{~S})$ peak data collected by the BABAR detector between January and June 2000, $\Delta m_{d}$ was measured to be,

$$
\Delta m_{d}=0.466 \pm 0.018 \pm 0.019 \mathrm{hps}^{-1}
$$
\end{abstract}

where the first error is statistical and the second is systematic. 


\section{Acknowledgments}

Firstly, I would like to thank my supervisor, Paul Dauncey, for his help, support and advice over the past three years. It was an honour and a pleasure to work with him. I would also like to thank Professor Peter Dornan for inviting me to join the HEP group. I acknowledge PPARC for funding this research.

I would also like to thank Jordan Nash (for trusting me with a million dollars worth of hardware). Phil Strother and Pete Sanders (lbw b. Symbian) for very helpful advice, comments and numerous cups of coffee.

Working for the BABAR collaboration was indeed a challenging and exciting experience. Whilst at SLAC I had the pleasure of working with an interesting and brilliant group of people. So, in no particular order, I would like to acknowledge, Helmut Marsiske, Ivo Eschrich, Jeff Olsen, John Back, James Wetheral, PierreAlexander Fischer, Stephen Gowdy, Rowan Hamilton, Chris O'Grady, Pete Elmer, Sumit Sen, Jim Cochran, Marco Serra and the members of the Dilepton mixing AWG.

A very special thank you to my friends both at SLAC and IC. In particular to the SLAC'ers: Dan A, Paul M, Phil S, John B, Robin E, Jim W, Gillian Wong, Wallom, Mickey G, Jon F, Jane T ..., and the bld. 28 tea ladies Mark and Pedro. Never shall I forget the "philosophical" debates we had whilst sipping our cafe latte (?!?). To my colleagues at IC: Brinick, Jeremy, Alex, Richard and Jon for sharing the experience of working in a room where the temperature was below freezing. To Dave Colling for installing DHCP. To John Hassard, for his continuing friendship and convincing me to do HEP.

I would like thank my family. To my mother, grandfather and father: thank you for all the support over the last three years. Finally, I would like to thank Virginia, Saina and Dyani for their support, encouragement and friendship over the past years. Thank you all.

London, December 2000. 


\section{Contents}

$\begin{array}{ll}\text { Abstract } & 2\end{array}$

$\begin{array}{ll}\text { Contents } & 4\end{array}$

$\begin{array}{lr}\text { List of Figures } & 8\end{array}$

$\begin{array}{ll}\text { List of Tables } & 11\end{array}$

$\begin{array}{ll}\text { Prologue } & 12\end{array}$

Chapter 1. Theoretical Overview 15

$\begin{array}{lll}1.1 & \text { Introduction } & 15\end{array}$

1.2 The Standard Model and spontaneous symmetry breaking 16

$\begin{array}{lll}1.3 & \text { The B meson system } & 19\end{array}$

1.3.1 Mixing in the B meson system 20

1.3.2 Time evolution of B meson states 23

1.3.3 Decays of the B meson states 25

1.4 Standard Model calculation of $M_{12}$ and $\Delta m_{d} \quad 26$

1.5 Experimental status of $\Delta m_{d} \quad 30$

$\begin{array}{lll}1.6 & \text { Chapter summary } & 31\end{array}$

Chapter 2. The BABAR Detector \& the PEP-II Storage Ring 33

2.1 Introduction 33

2.2 PEP-II storage ring 33

$\begin{array}{lll}2.2 .1 & \text { Introduction } & 33\end{array}$

$\begin{array}{lll}\text { 2.2.2 The main storage ring } & 34\end{array}$ 
2.2.3 The PEP-II interaction region

2.2.4 The PEP-II beam spot

2.3 The BABAR detector 35

2.3.1 The BABAR Detector Coordinate System 36

2.3.2 Silicon Vertex Tracker (SVT) 37

2.3.3 Drift Chamber (DCH) 40

2.3.4 Detector of Internally Reflected Čerenkov Radiation (DIRC) 43

2.3.5 Electromagnetic Calorimeter (EMC) 46

2.3.6 Instrumented Flux Return (IFR) 50

$\begin{array}{lll}2.3 .7 & \text { The trigger system } & 53\end{array}$

2.4 Chapter summary $\quad 54$

Chapter 3. The calorimeter readout system and simulation $\quad 55$

3.1 Introduction 55

$\begin{array}{lll}3.2 & \text { BABAR DAQ system } & 56\end{array}$

$\begin{array}{lll}3.2 .1 & \text { Introduction } & 56\end{array}$

$\begin{array}{lll}3.2 .2 & \text { DAQ architecture } & 56\end{array}$

3.3 The EMC front end electronics and DAQ system 60

$\begin{array}{lll}3.3 .1 & \text { EMC electronics }\end{array}$

$\begin{array}{lll}\text { 3.3.2 The UPC ROM } & 61\end{array}$

3.4 The EMC front end electronics simulation 65

$\begin{array}{lll}3.4 .1 & \text { The BABAR simulation } & 65\end{array}$

$\begin{array}{lll}3.4 .2 & \text { EMC simulation } & 66\end{array}$

3.4.3 Machine background noise in the simulation 68

$\begin{array}{lll}\text { 3.4.4 Digital filter feature extraction } & 72\end{array}$

$\begin{array}{lll}3.5 & \text { Chapter summary } & 78\end{array}$

$\begin{array}{lll}\text { Chapter 4. } & \text { Dilepton analysis and event selection } & 80\end{array}$

4.1 Introduction 80

4.2 Dilepton analysis method 80

4.3 Event sample $\quad 82$

4.4 Multi-Hadron Selection 83 
4.5 Track quality cuts 84

4.6 Lepton identification 85

$\begin{array}{lll}\text { 4.6.1 Electron identification } & 85\end{array}$

$\begin{array}{lll}\text { 4.6.2 Electron identifier performance } & 87\end{array}$

$\begin{array}{lll}\text { 4.6.3 } & \text { Muon identification } & 88\end{array}$

$\begin{array}{lll}\text { 4.6.4 Muon identifier performance } & 91\end{array}$

$\begin{array}{lll}4.7 & \text { Background reduction } & 92\end{array}$

$\begin{array}{lll}\text { 4.7.1 Cascade leptons } & 92\end{array}$

$\begin{array}{lll}\text { 4.7.2 Continuum backgrounds } & 93\end{array}$

$\begin{array}{lll}4.7 .3 \boldsymbol{J} / \boldsymbol{\psi} \text { backgrounds } & 95\end{array}$

$\begin{array}{lll}\text { 4.7.4 Gamma conversion backgrounds } & 97\end{array}$

$\begin{array}{lll}\text { 4.7.5 } & \text { Bhabha and } \gamma \gamma \text { backgrounds } & 97\end{array}$

$\begin{array}{lll}\text { 4.8 } & \text { SVT z layer hits } & 98\end{array}$

$\begin{array}{llr}4.9 & \text { Selection of primary leptons } & 99\end{array}$

$\begin{array}{ll}4.10 \text { Selected event sample } & 100\end{array}$

$\begin{array}{ll}4.11 \text { Chapter summary } & 100\end{array}$

Chapter 5. Extraction of $\Delta m_{d}$ and a study of the systematics $\quad 101$

$\begin{array}{lll}5.1 & \text { Introduction } & 101\end{array}$

$\begin{array}{lll}5.2 & \text { Estimation of the B decay vertex } & 102\end{array}$

$\begin{array}{lll}\text { 5.2.1 Calculation of } \Delta z \text { and } \Delta t & 102\end{array}$

$\begin{array}{lll}5.3 & \Delta \mathrm{z} \text { resolution } & 103\end{array}$

$\begin{array}{ll}\text { 5.3.1 Global resolution function } & 104\end{array}$

$\begin{array}{lll}\text { 5.3.2 Event error resolution function } & 104\end{array}$

$\begin{array}{lll}5.4 & \text { Discussion of the fit procedure } & 106\end{array}$

$\begin{array}{lll}\text { 5.4.1 Method of maximum likelihood } & 106\end{array}$

$\begin{array}{lll}\text { 5.4.2 Fit procedure for backgrounds } & 107\end{array}$

$\begin{array}{lll}5.5 & \text { Background characterisations } & 108\end{array}$

$\begin{array}{lll}\text { 5.5.1 Cascade backgrounds } & 109\end{array}$

$\begin{array}{lll}\text { 5.5.2 } & B^{+} B^{-} \text {backgrounds } & 112\end{array}$

$\begin{array}{lll}\text { 5.5.3 Continuum backgrounds } & 113\end{array}$

$\begin{array}{lll}\text { 5.5.4 Other backgrounds } & 114\end{array}$ 
5.6 Measurement of $\Delta m_{d} \quad 114$

$\begin{array}{lll}\text { 5.6.1 Probability density functions } & 115\end{array}$

$\begin{array}{ll}\text { 5.6.2 Validation of the fit } & 117\end{array}$

$\begin{array}{lll}\text { 5.6.3 Data fit results } & 118\end{array}$

$\begin{array}{lll}5.7 & \text { Systematic uncertainties } & 119\end{array}$

$\begin{array}{lll}5.7 .1 & B^{0} \text { lifetime } & 119\end{array}$

$\begin{array}{llr}5.7 .2 & B^{ \pm} \text {lifetime } & 120\end{array}$

$\begin{array}{lll}\text { 5.7.3 Resolution function parameters } & 120\end{array}$

$\begin{array}{ll}\text { 5.7.4 Global resolution function } & 122\end{array}$

$\begin{array}{lll}\text { 5.7.5 Lepton mis-identification } & 123\end{array}$

$\begin{array}{lll}\text { 5.7.6 } & \text { Backgrounds characterisation systematics } & 123\end{array}$

$\begin{array}{lll}\text { 5.7.7 } & \text { Background fractions } & 124\end{array}$

$\begin{array}{lll}5.7 .8 & \text { Boost approximation } & 124\end{array}$

$\begin{array}{lll}5.7 .9 & \text { Beamspot parameters } & 125\end{array}$

$\begin{array}{ll}\text { 5.7.10 Simulation deficiencies } & 125\end{array}$

$\begin{array}{ll}\text { 5.7.11 Summary of systematic errors } & 125\end{array}$

$\begin{array}{lll}5.8 & \text { Consistency checks } & 125\end{array}$

$\begin{array}{lll}5.9 & \text { Chapter summary } & 127\end{array}$

$\begin{array}{lll}\text { Chapter 6. Conclusions } & 131\end{array}$

Appendix A. Second order polynomial fit to an EmcWaveform 134

Appendix B. Analytical form of the convoluted lifetime pdf 136

Appendix C. Analytical forms of the convoluted unmixed and mixed $\begin{array}{ll}\text { pdfs } & 138\end{array}$

$\begin{array}{ll}\text { References } & 144\end{array}$ 


\section{List of Figures}

1.1 The Unitarity triangle. 20

$\begin{array}{lll}1.2 & \text { Neutral B mixing box diagram. } & 27\end{array}$

1.3 The $\Delta B=2$, four quark operator. 28

1.4 ICHEP $2000 \Delta m_{d}$ average for BABAR, LEP, SLD and CDF. 30

1.5 Unitarity triangle in the $(\rho, \eta)$ plane. 31

2.1 The PEP-II interaction region. 36

2.2 The BABAR Detector. 37

2.3 Cross sectional view of the BABAR silicon vertex tracker in the yz plane. 38

2.4 Cross sectional view of the BABAR silicon vertex tracker in the $\mathrm{r} \phi$ plane. 39

2.5 Position resolution of the BABAR silicon vertex tracker. 41

2.6 The axial and stereo superlayers of the BABAR drift chamber. 42

$2.7 \mathrm{dE} / \mathrm{dX}$ measurements from the BABAR drift chamber. 43

2.8 The $p_{\mathrm{t}}$ resolution in the BABAR drift chamber. 44

2.9 A schematic view of the BABAR DIRC. 45

2.10 Production of Čerenkov radiation in the BABAR DIRC. 46

2.11 Cerenkov angle resolution of the BABAR DIRC. 47

2.12 A cross sectional view of the BABAR EMC. 49

2.13 Energy resolution for the BABAR EMC. 50

2.14 Position resolution for the BABAR EMC. 51

2.15 Resistive Plate Capacitors (RPCs) used by the BABAR IFR. 52

2.16 Level 3 display showing a multi-hadronic event. 54

3.1 The full assembly of the BABAR DAQ system. 57 
3.2 A schematic digram of the BABAR ROM.

3.3 The EMC readout chain showing the connections from the front end to the ROMs.

3.4 The format of data packets received by the BABAR UPC ROM.

3.5 A picture of the BABAR UPC ROM.

3.6 The BABAR UPC ROM intermediate store.

3.7 Functional diagram of the EmcSim package.

3.8 Level 1 time used in the BABAR simulation.

3.9 The GHit mixing procedure as implemented for the EMC. 70

3.10 The event mixing procedure as implemented for the EMC. 71

3.11 GHit mixing - event mixing, digi number and time comparison plots. $\quad 73$

3.12 GHit mixing - event mixing digi energy comparison plot. 74

3.13 The difference in the EmcGHit energy and the digi energy. 75

3.14 Filtered energy distribution. $\quad 76$

3.15 The difference in the EmcGHit energy and the fitted digi energy. $\quad 77$

3.16 The energy resolution before and after the polynomial fit. 78

3.17 the difference between the EmcGHit time and the digi time. 79

4.1 The production of primary and cascade leptons from the semileptonic decays of the b and c quarks.

4.2 Discriminating variables used by the electron selector. 85

4.3 Efficiency and purity distributions for the electron selector. 88

4.4 Discriminating variables used by the muon selector. 89

4.5 Efficiency and purity plots for the muon selector. 91

4.6 The production of the two types of cascade leptons. 93

4.7 CMS momentum spectra for the primary and cascade leptons. 94

4.8 The normalised second Fox-Wolfram moment distributions for B0 and $\begin{array}{ll}\text { continuum MC. } & 95\end{array}$

4.9 MC CMS momentum spectra for primary leptons and leptons from $J / \psi$ $\begin{array}{ll}\text { decays. } & 96\end{array}$

$4.10 \mathrm{MC} J / \psi$ mass spectra for decays to electron and muon pairs. $\quad 97$

4.11 MC invariant mass spectrum for gamma conversions. 98 
5.1 POCA approach to used to estimate the positions of the B decay vertices.102

5.2 MC global $\Delta z$ resolution function. 104

5.3 MC event error resolution function. 105

5.4 Data and MC comparison for the resolution function for ee and $\mu \mu$ events.107

5.5 Mixed MC $D^{ \pm}$cascade background parameterisation. 109

5.6 Unmixed MC $D^{ \pm}$cascade background parameterisation. 110

5.7 Mixed MC $D^{0}$ cascade background parameterisation. 111

5.8 Unmixed MC $D^{0}$ cascade background parameterisation. 111

5.9 Mixed MC $B^{ \pm}$background parameterisation. 112

5.10 Unmixed MC $B^{ \pm}$background parameterisation. 113

5.11 Mixed MC $c \bar{c}$ background parameterisation. 113

5.12 Unmixed MC $c \bar{c}$ background parameterisation. 114

5.13 Mixed and unmixed off-resonance data and continuum MC comparison. 115

5.14 Unmixed and mixed $|\Delta z|$ distributions for primary leptons. 118

5.15 Unmixed and mixed $|\Delta z|$ distributions for selected $B^{0} \bar{B}^{0}$ events. 119

5.16 Unmixed and mixed $|\Delta z|$ distribution for a sample of selected $B^{0} \bar{B}^{0}$, $\begin{array}{ll}B^{+} B^{-} \text {and } c \bar{c} \text { events. } & 120\end{array}$

5.17 Unmixed and mixed $|\Delta z|$ distributions for data. 121

5.18 Asymmetry distribution. 122

$5.19 \Delta m_{d}$ results for lepton sub-samples. $\quad 127$

$5.20 \Delta m_{d}$ results for data sub-samples. 128

$5.21 \Delta m_{d}$ results for different lower bounds on the $p^{*}$ cut. 129

5.22 A summary of the $\Delta m_{d}$ results to date. 130 


\section{List of Tables}

2.1 PEP-II parameters.

2.2 Properties of the Thallium doped CsI crystals used by the BABAR EMC. 48

4.1 Event sample used in the analysis.

4.2 Data sub-sample luminosities.

4.3 Electron selector efficiency and purity table.

4.4 Muon selector efficiency and purity table.

4.5 Selection efficiencies for the CMS momentum cut. 94

4.6 Quark pair production cross sections at the $\Upsilon(4 \mathrm{~S}) . \quad 95$

4.7 The selection efficiencies obtained with the normalised second Fox-Wolfram moment cut.

4.8 Selection efficiencies with the aplanarity cut.

4.9 Selection efficiencies with the cut on SVT z hits.

4.10 A summary of the selected event sample used for the extraction of $\Delta m_{d} . \quad 100$

5.1 Correlation matrix for the global resolution function fit parameters.

5.2 Correlation matrix for the event error resolution function fit parameters. 106

5.3 Systematic errors due to the resolution function parameters.

5.4 Systematic errors due to cascade background characterisations.

5.5 Systematic errors due to $B^{+} B^{-}$background characterisations.

5.6 Systematic errors due to continuum background characterisations.

5.7 Systematic errors due to background fractions.

5.8 A summary of the systematic errors. 


\section{Prologue}

The drive of humanity to explain the unexplained, explore the unexplored has been a prevailing feature throughout time. This drive led to the postulation of theories or models that tried to explain certain characteristics of the world. These models were not always founded on scientific fact. Many were pure speculation, some philosophical and others, theological. Not all ideas or theories proved to be valid. For every correct interpretation, there were many more incorrect ones. Each idea, model or theory brought with them a new set of questions and puzzles. This process of questions, answers and yet more questions is what has helped humanity expand its knowledge of the universe. What drives this desire of humanity for intellectual stimulus and understanding is open to debate. But whatever it is, it's this mysterious "force" that has given rise to modern day scientific reasoning and methodologies.

The present day study of particle physics has its roots in the work carried out by Democritus in the 5th century B.C. He proposed the atomic structure of matter by expanding on the ideas put forward by his mentor, Leucippus. The atom was thus considered to be the basic building block of all matter. Work carried by Rutherford in the early $19^{\text {th }}$ century showed that the atom is not fundamental but consists of a positively charged core (nucleus) which is surrounded by negatively charged particles (electrons). Study of the atoms and the subsequent splitting of the nucleus showed that the nucleus is not a fundamental object but it too is made up of smaller particles; the positively charged proton and the electrically neutral neutron. During the middle part of the last century, the first evidence was seen to suggest that protons and neutrons are not fundamental objects but are made up of point-like particles known as quarks. This period was also the golden years of particle physics. Hundreds of new and exotic particles were discovered. With these new discoveries, 
a theory or model was required to explain the origin of these particles and the Standard Model (SM) of particle physics was thus born.

The SM is an attempt to explain the origin and interactions of all observed (and some unobserved) particles. The model, as it stands, predicts the following; all matter is made up of point-like objects known as quarks and leptons. The quarks come in six "flavours"; up, down, strange, charm, bottom and top in order of increasing mass and are structured in three "generations". The massive leptons and their massless (?) neutrino partners are also divided into three such "generations". All interactions between these particles can be described by four fundamental forces; strong, weak, electromagnetic and gravity. Due to its weakness, gravity plays a very minor role within particle accelerators. In order to test the validity of a model, its parameters and predictions have to be tested and verified by experimental evidence. This is where the success of the SM lies. Almost all its predictions have been tested and verified to a very high precision. But, nevertheless, it is very likely that the $\mathrm{SM}$ is not the final story. Just as Newtonian mechanics is an approximation to general relativity and quantum mechanics, it is probable that the SM too is an approximation of a more complex but yet elegant theory.

The next few years are clearly going to be a very exciting time for particle physics. The dawn of the new century has seen the commissioning and the first data from the "B Factories", BABAR and Belle. The search for the elusive Higgs Boson, which seems to have just been outside the clutches of the present LEP experiments, will take a new turn with the next generation of experiments at Fermilab and the Large Hadron Collider (LHC) at CERN. The results from these might serve as a further verification of the SM or, more interestingly, give us new physics and a glimpse of that final theory.

The rest of the thesis is in six chapters. The theoretical background required for the measurement of the $\Delta m_{d}$ will be presented in the next chapter. This will include an overview of the spontaneous symmetry breaking within the SM, the CabibboKobayashi-Maskawa (CKM) mixing matrix and the Unitarity triangle. This will be followed by a discussion of the mechanics of the B meson system. The SM calculation of $\Delta m_{d}$ will then be presented. The chapter will end with a summary of the experimental status of $\Delta m_{d}$ and the constraints on the Unitarity triangle. 
An introduction into the BABAR experiment and the PEP-II collider ring will be given in chapter 2 . This will include an overview of the BABAR sub-detector components and a summary of the performances achieved.

Chapter 3 is devoted to the BABAR electromagnetic calorimeter (EMC). The chapter will begin with an overview of the data acquisition software employed by the BABAR experiment. This will be followed by a discussion on the functionality that is specific to the EMC. In particular, the untriggered personality card read out modules used by the EMC will be described. The EMC front end electronics simulation software will then be discussed. This will include an overview of the digital filter algorithms used by the simulation and a discussion on the implementation of machine background noise within the simulation environment.

The dilepton method as implemented by the BABAR experiment will be introduced in chapter 4. This will contain an overview of the lepton identification algorithms used. Following a summary of the Monte Carlo (MC) and data samples used, a discussion on the possible sources of backgrounds and the selection cuts used to reduce the contamination from such backgrounds will be presented. The chapter will end with a summary of the obtained selection efficiencies.

The fitting procedure used to extract $\Delta m_{d}$ from the data will be presented in chapter 5 . The method used to estimate the $\mathrm{B}$ decay vertex positions along with a summary of the obtained resolutions will be given. This will be followed by the parameterisation used to characterise the non-negligible residual backgrounds present in the analysis. The result of the fit and a summary of the systematic effects will then be given. The stability of the fit result will then be discussed.

The thesis will then end with a short summary and conclusions in chapter 6 


\section{Chapter 1}

\section{Theoretical Overview}

\subsection{Introduction}

The SM of particle physics has had incredible success in describing the interactions of matter. Its predictions have been tested and verified to a very high precision. Nevertheless, two rather important issues remain outstanding; the origin of mass (and the resulting Higgs boson) and the origin of $\mathrm{CP}$ violation. The primary aims of current generation B factory experiments such as BABAR and Belle are to address the latter issue and to do a systematic study of $\mathrm{CP}$ violation in the B meson system. $\mathrm{CP}$ violation has already been observed and studied in the $\mathrm{K}$ meson system [1]. The $\epsilon_{K}$ parameter has been measured by various experiments. The measurements made in the $\mathrm{K}$ meson system have been used to constrain the parameters of the CabibboKobayashi-Maskawa (CKM) matrix but they do not provide a full test of the SM. We may find that the measurements made by BABAR and Belle are consistent with the predictions of the SM or they maybe such that it would not be possible to find a consistent set of CKM parameters. The latter case is an indication of physics beyond the minimal SM. It should be noted that any extension of the present SM will need to have $\mathrm{CP}$ violating effects built into it. $\mathrm{CP}$ violation has rather interesting consequences from a cosmological point of view. Indeed, the matter anti-matter asymmetry that is observed in the present universe has been attributed to CP violation [2]. However, the SM does not produce enough asymmetry to describe the observed matter anti-matter imbalance $[3,4,5]$ and other sources of $\mathrm{CP}$ violation have to be investigated [6]. 
The above experiments, which run on the $\Upsilon(4 \mathrm{~S})$ in a $\mathrm{B}$ factory mode, will also address $\mathrm{B}$ physics issues and do precision measurements on the $B_{d}$ system. In particular, precision measurements of the B lifetime and the $B_{d}$ mixing parameter, $\Delta m_{d}$, will be made. It is expected that measurements made on the B system by these experiments will allow a further verification of the SM predictions.

The aim of this chapter is to give an overview of the theoretical framework required to study $\mathrm{B}$ meson physics, including $\mathrm{B}$ meson mixing. An introduction to the electroweak SM will be given. This section will introduce the SM Lagrangian, Spontaneous Symmetry Breaking (SSB) of the Higgs field and the CKM matrix. The mechanics of the B meson system will then be considered. This will include a discussion of the mixing, time evolution and subsequent decays of the $\mathrm{B}$ meson states. The SM calculation of $\Delta m_{d}$ will then be presented. The section will end with a summary of the experimental status of $\Delta m_{d}$.

\subsection{The Standard Model and spontaneous sym- metry breaking}

The renormalizable, $S U(3)_{C} \otimes S U(2)_{L} \otimes U(1)_{Y}$ SM Lagrangian can be written in terms of four terms: the kinetic term $\left(\mathcal{L}_{K}\right)$, the Higgs term $\left(\mathcal{L}_{H}\right)$, the Yukawa couplings $\left(\mathcal{L}_{Y}\right)$ and a potential, $V(\phi)$, such that, [7] [8]:

$$
\mathcal{L}=\mathcal{L}_{K}+\mathcal{L}_{H}+\mathcal{L}_{Y}-V(\phi)
$$

The kinetic term, $\mathcal{L}_{K}$, is given by,

$$
\mathcal{L}_{K}=i \bar{Q}_{L} \gamma^{\mu} D_{\mu} Q_{L}
$$

where $Q_{L}$ is a left handed quark doublet. The covariant derivative $\left(D_{\mu}\right)$, which is required for gauge invariance, is given by,

$$
D^{\mu}=\partial^{\mu}+i g_{s} G_{a}^{\mu} L_{a}+i g W_{b}^{\mu} T_{b}+i g^{\prime} B^{\mu} Y
$$

The $G_{a}^{\mu}$ are the eight gluon fields. The $W_{b}^{\mu}$ are the three weak interaction bosons and $B^{\mu}$ is the hypercharge boson. The $L_{a}$ and $T_{b}$ are the generators for $S U(3)_{C}$ and $S U(2)_{L}$ and the $U(1)$ hypercharges are given by $Y$. 
The contribution to the Lagrangian from the scalar Higgs field, $\phi$, is given by,

$$
\mathcal{L}_{H}=\mu^{2} \phi^{\dagger} \phi-\lambda\left(\phi^{\dagger} \phi\right)^{2}
$$

The Yukawa coupling term for the quarks can be written as [9]

$$
\mathcal{L}_{Y}=Y_{u} \bar{Q}_{L} \tilde{\phi} u_{R}+Y_{d} \bar{Q}_{L} \phi d_{R}+\text { h.c. }
$$

where $\tilde{\phi}$ is the conjugate representation of $\phi, u_{R}$ and $d_{R}$ are the right handed quark singlets. The $L$ and $R$ refer to the left and right handed states respectively. It should be noted that, for simplicity, the leptonic contribution has been left out in the above equation. This Yukawa part of the Lagrangian is, in general, CP violating and it can be shown that CP is violated if and only if [10]

$$
\mathcal{I} m\left(\operatorname{det}\left[\mathrm{Y}_{\mathrm{d}} \mathrm{Y}_{\mathrm{d}}^{\dagger}, \mathrm{Y}_{\mathrm{u}} \mathrm{Y}_{\mathrm{u}}^{\dagger}\right]\right) \neq 0
$$

The quark doublet in the above Lagrangian can be written as,

$$
Q_{L}=\left(\begin{array}{l}
u^{(0)} \\
d^{(0)}
\end{array}\right)_{L}
$$

The weak charged current interaction Lagrangian for these states is then given by,

$$
\mathcal{L}^{C C}=\frac{-g}{\sqrt{2}} W^{+\mu} \sum_{i} \bar{u}_{i L}^{(0)} \gamma_{\mu} d_{i L}^{(0)}+\text { h.c. }
$$

With the Spontaneous Symmetry Breaking (SSB) of the above Lagrangian and $\phi$ acquiring its vacuum expectation value, $\mathcal{L}_{Y}$ becomes the mass terms for the quarks.

$$
\mathcal{L}_{Y} \longrightarrow \mathcal{L}_{\text {mass }}=\sum_{q=u, d} \bar{q}_{i L} M_{i j}^{(q)} q_{j R}
$$

The mass matrices, $M^{(q)}$, that couple the quarks are given by,

$$
M_{i j}^{(q)}=\frac{v}{\sqrt{2}} Y_{i j}^{(q)}
$$

The vacuum expectation value of the scalar field $\phi$, is denoted by $v$ [11]. From equation 1.9 it can be seen that the mass matrices mix the weak eigenstates of 
different generations. The mass matrices in equation 1.10 can in fact be diagonalised by means of unitarity transformation matrices, V.

$$
M_{\text {diag }}^{q}=V_{L}^{(q)} M^{(q)} V_{R}^{(q) \dagger}
$$

The eigenstates of the mass matrices (mass eigenstates) can now be expressed in terms of the unitary transformation matrices and the original massless weak eigenstates defined in equation 1.7 as,

$$
\begin{aligned}
& u_{L, R}=V_{L, R}^{(u)} u_{L, R}^{(0)} \\
& d_{L, R}=V_{L, R}^{(d)} d_{L, R}^{(0)}
\end{aligned}
$$

The charged current Lagrangian given in equation 1.8 can now be expressed in terms of the mass eigenstates and the unitarity matrices as,

$$
\mathcal{L}^{C C}=\frac{-g}{\sqrt{2}} W^{+\mu}\left(\bar{u}_{L} \gamma_{\mu} V_{L}^{(u) \dagger} V_{L}^{(d)} d_{L}\right)+\text { h.c. }
$$

The product of the two matrices, $V_{L}^{(u) \dagger}$ and $V_{L}^{(d)}$ gives the famous CKM mixing matrix $[12]$.

$$
V_{C K M}=V_{L}^{(u) \dagger} V_{L}^{(d)}=\left(\begin{array}{ccc}
V_{u d} & V_{u s} & V_{u b} \\
V_{c d} & V_{c s} & V_{c b} \\
V_{t d} & V_{t s} & V_{t b}
\end{array}\right)
$$

This matrix has many different representations, the one below is due to Harari \& Leurer [13].

$$
V_{C K M}=\left(\begin{array}{ccc}
c_{12} c_{13} & s_{12} c_{13} & s_{13} e^{-i \delta} \\
-s_{12} c_{23}-c_{12} s_{23} s_{13} e^{i \delta} & c_{12} c_{23}-s_{12} s_{23} s_{13} e^{i \delta} & s_{23} c_{13} \\
s_{12} s_{23}-c_{12} c_{23} s_{13} e^{i \delta} & -c_{12} s_{23}-s_{12} c_{23} s_{13} e^{i \delta} & c_{23} c_{13}
\end{array}\right)
$$

where, $c_{i j}=\cos \theta_{i j}, s_{i j}=\sin \theta_{i j}$ for $(i, j=1,2,3)$ and $\theta_{i j}$ are real angles and $\delta$ is a complex phase factor. It was shown by Kobayashi and Maskawa in 1973 that CP violation occurs in the SM with three particle generations due to the presence of this non-trivial phase [12]. The above representation of the CKM matrix can be rewritten in the standard Wolfenstein parameterisation [14]. In doing this, the elements of the 
CKM matrix are expanded in a power series in terms of the parameter $\lambda=\left|V_{u s}\right|$ to give,

$$
V_{C K M} \simeq\left(\begin{array}{ccc}
1-\frac{\lambda^{2}}{2} & \lambda & A \lambda^{3}(\rho-i \eta) \\
-\lambda & 1-\frac{\lambda^{2}}{2} & A \lambda^{2} \\
A \lambda^{3}(1-\rho-i \eta) & -A \lambda^{2} & 1
\end{array}\right)+\mathcal{O}\left(\lambda^{4}\right)
$$

Here, $s_{12}=\lambda, s_{23}=A \lambda^{2}, s_{13}=A \sqrt{\rho^{2}+\eta^{2}} \lambda^{3}$ and $\delta=\tan ^{-1}\left(\frac{\eta}{\rho}\right)$. The unitarity nature of the CKM matrix can be exploited to yield nine unitarity relations [15]. Of these, the one that is of interest to us for the $B_{d}$ meson system is,

$$
V_{u d} V_{u b}^{*}+V_{c d} V_{c b}^{*}+V_{t d} V_{t b}^{*}=0
$$

Geometrically, the above is an equation of a triangle in the complex plane. This triangle, most commonly known as the Unitarity Triangle, is shown in figure 1.1. The three angles, $\alpha, \beta$ and $\gamma$ of the above triangle can be defined as,

$$
\begin{aligned}
& \alpha=\arg \left(-\frac{V_{t d} V_{t b}^{*}}{V_{u d} V_{u b}^{*}}\right) \\
& \beta=\arg \left(-\frac{V_{c d} V_{c b}^{*}}{V_{t d} V_{t b}^{*}}\right) \\
& \gamma=\arg \left(-\frac{V_{u d} V_{u b}^{*}}{V_{c d} V_{c b}^{*}}\right)
\end{aligned}
$$

Thus, the only way in which CP violation can be described in the $S U(2)_{L} \otimes U(1)_{Y}$ Weinberg-Salam [16] electroweak model with a single Higgs doublet is via complex Yukawa couplings between the fermions and the Higgs boson. As was shown, after the $S U(2)_{L} \otimes U(1)_{Y}$ symmetry is broken, the Yukawa interactions became the mass matrices for the quarks.

\subsection{The B meson system}

The theoretical framework required to study the B meson system will be presented in this section. The section will begin with the phenomenology of mixing in the B system and will include the definition of $\Delta m_{d}$. The time evolution of the mixed and unmixed states will then be considered. A few words will then be said about the quantum mechanical coherence of the $\Upsilon(4 \mathrm{~S})$ state. This will be followed by an overview of the mechanics of $\mathrm{B}$ meson decay. 

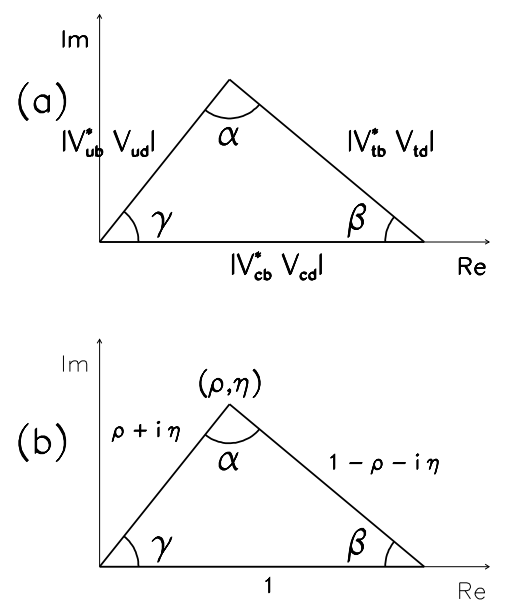

Figure 1.1: The Unitarity triangle. Figure (a) depicts the triangle in terms of the CKM elements of equation (9). Figure (b) is the triangle in the Wolfenstein parameterisation.

\subsubsection{Mixing in the B meson system}

The aim of this section is to give a model independent account of mixing in the B meson system. This will be accomplished by considering the quantum mechanics of a two body system. The results that are obtained for the generalised system will then be applied to the specialised case of mixing in the B system.

Consider an arbitrary quantum mechanical state, $\psi$. Within the standard framework of quantum mechanics, the above state can be expanded in terms of its eigenstates. If the eigenstates of $\psi$ are given by $\psi_{a}$ and $\psi_{b}, \psi$ can be expressed as,

$$
\psi=\psi_{a}|a\rangle+\psi_{b}|b\rangle
$$

The time evolution of the above state is governed by the time dependent Schrödinger equation,

$$
i \frac{d}{d t}\left(\begin{array}{c}
\psi_{a} \\
\psi_{b}
\end{array}\right)=\mathcal{H}\left(\begin{array}{c}
\psi_{a} \\
\psi_{b}
\end{array}\right)
$$

with the form of the non-Hermitian Hamiltonian, $\mathcal{H}$, being constrained by the CPT symmetry [17] [18]. The Hamiltonian can in fact be written in terms of two 
Hermitian components; the so called Mass matrix $(\mathcal{M})$ and the Disintegration matrix $(\Gamma)$ such that,

$$
\mathcal{H}=\mathcal{M}-\frac{i}{2} \Gamma
$$

The mass eigenstates, of the $\mathrm{B}$ meson system, $B_{L}^{0}$ and $B_{H}^{0}$ can be expressed in terms of the flavour eigenstates, $B^{0}$ and $\bar{B}^{0}$. The $B_{L}^{0}$ and the $B_{H}^{0}$ which are referred to as the "light" and "heavy" states respectively can be expanded in terms of the flavour eigenstates as (cf. equation 1.20),

$$
\begin{aligned}
& \left|B_{L}^{0}\right\rangle=p\left|B^{0}\right\rangle+q\left|\bar{B}^{0}\right\rangle \\
& \left|B_{H}^{0}\right\rangle=p\left|B^{0}\right\rangle-q\left|\bar{B}^{0}\right\rangle
\end{aligned}
$$

with the parameters $p$ and $q$ subject to the normalisation condition,

$$
|p|^{2}+|q|^{2}=1
$$

Again, the evolution of the $B_{H}$ and the $B_{L}$ eigenstates are governed by the time dependent Schrödinger equation. As before, the Hamiltonian representing the evolution can be expressed in terms of the corresponding mass and disintegration matrices. The mass matrix is in fact due the processes involving virtual intermediate states. The elements of this matrix can be calculated using perturbation theory and for the B system they are given by,

$$
M_{i j}=M_{B} \delta_{i j}+\left\langle i\left|\mathcal{T}^{(\Delta B=2)}\right| j\right\rangle+\Sigma_{v} \frac{\left\langle i\left|\mathcal{T}^{(\Delta B=1)}\right| v\right\rangle\left\langle v\left|\mathcal{T}^{(\Delta B=1)}\right| j\right\rangle}{\left(M_{B}-E_{v}\right)}
$$

where $|v\rangle$ is the intermediate virtual state, $\mathcal{T}$ is a transition matrix that connects the two states with different Beauty quantum number, $\mathrm{B}$, and $E_{v}$ is the energy of the virtual state. The diagonal elements, $M_{11}$ and $M_{22}$ are due to the masses and the binding energies of the quarks within the B mesons. $M_{12}$ and $M_{21}$ are terms that arise due to the mixing between the $B^{0}$ and the $\overline{B^{0}}$. As will be shown, $M_{12}$ is in fact proportional to the $B_{d}$ mixing parameter, $\Delta m_{d}$. The Disintegration matrix, $\Gamma$, is due to intermediate real states $(|f\rangle)$ and is given by, 


$$
\Gamma_{i j}=2 \pi \Sigma_{f} C_{f}\left\langle i\left|\mathcal{T}^{(\Delta B=2)}\right| f\right\rangle\left\langle f\left|\mathcal{T}^{(\Delta B=2)}\right| j\right\rangle
$$

with $C_{f}$ being a phase factor.

The mass and the width differences between the two mass eigenstates are defined as,

$$
\begin{aligned}
\Delta m_{d} & =M_{H}-M_{L} \\
\Delta \Gamma_{B} & =\Gamma_{H}-\Gamma_{L}
\end{aligned}
$$

Solving the eigenvalue equation, it can be shown that,

$$
\begin{aligned}
\Delta m_{d}^{2}-\frac{1}{4}\left(\Delta \Gamma_{B}\right)^{2} & =4\left(\left|M_{12}\right|^{2}-\frac{1}{4}\left|\Gamma_{12}\right|^{2}\right) \\
\Delta m_{d} \Delta \Gamma_{B} & =4 \mathcal{R} e\left(M_{12} \Gamma_{12}^{*}\right)
\end{aligned}
$$

and

$$
\frac{q}{p}=-\frac{2 M_{12}^{*}-i \Gamma_{12}^{*}}{\Delta m_{d}-\frac{i}{2} \Gamma_{12}}=-\frac{\Delta m_{d}-\frac{i}{2} \Delta \Gamma_{B}}{2 M_{12}-i \Gamma_{12}}
$$

In the B system, using the observation that $\left|\Gamma_{12}\right|<<\left|M_{12}\right|$, this gives,

$$
\begin{aligned}
\Delta m_{d} & =2\left|M_{12}\right| \\
\Delta \Gamma_{B} & =\frac{2 \mathcal{R} e\left(M_{12} \Gamma_{12}^{*}\right)}{M_{12}}
\end{aligned}
$$

and to leading order, the ratio $\left(\frac{q}{p}\right)$ can be written as,

$$
\frac{q}{p}=-\frac{M_{12}^{*}}{\left|M_{12}\right|}\left[1-\frac{1}{2} \mathcal{I} m\left(\frac{\Gamma_{12}}{M_{12}}\right)\right]
$$

As seen in equation 1.33, a calculation of the mixing parameter therefore involves a calculation of the matrix element, $M_{12}$. This is addressed in section 1.4. 


\subsubsection{Time evolution of B meson states}

The mechanics of the evolution of the B meson states will now be studied. Consider an initial pure B state; a state that is an equal mixture of the two mass eigenstates, $B_{H}^{0}$ and $B_{L}^{0}$ described above. Such a state can be expressed as,

$$
\left|B^{0}(0)\right\rangle=\frac{\left|B_{L}^{0}\right\rangle+\left|B_{H}^{0}\right\rangle}{2 p}
$$

The time evolution of a state such as this is governed by the time dependent Schrödinger equation. Denoting the Hamiltonians for the heavy and light states as $\mathcal{H}_{H}$ and $\mathcal{H}_{L}$ respectively, the state vector at time t is given by [19],

$$
\begin{aligned}
\left|B^{0}(t)\right\rangle & =\frac{1}{2 p}\left(e^{-i \mathcal{H}_{L} t}\left|B_{L}^{0}\right\rangle+e^{-i \mathcal{H}_{H} t}\left|B_{H}^{0}\right\rangle\right) \\
& =\frac{1}{2 p}\left[e^{-i \mathcal{H}_{L} t}\left(p\left|B^{0}(0)\right\rangle+q\left|\bar{B}^{0}(0)\right\rangle\right)+e^{-i \mathcal{H}_{H} t}\left(p\left|B^{0}(0)\right\rangle-q\left|\bar{B}^{0}(0)\right\rangle\right)\right]
\end{aligned}
$$

So:

$$
\left|B^{0}(t)\right\rangle=f_{+}(t)\left|B^{0}(0)\right\rangle+\frac{q}{p} f_{-}(t)\left|\bar{B}^{0}(0)\right\rangle
$$

where,

$$
\begin{aligned}
& f_{+}(t)=e^{-i \frac{\left(M_{L}+M_{H}\right)}{2} t} e^{-\gamma t / 2} \cos \left(\frac{\Delta m_{d} t}{2}\right) \\
& f_{-}(t)=e^{-i \frac{\left(M_{L}+M_{H}\right)}{2} t} e^{-\gamma t / 2} i \sin \left(\frac{\Delta m_{d} t}{2}\right)
\end{aligned}
$$

and,

$$
\gamma=\left(\Gamma_{L}+\Gamma_{H}\right) / 2
$$

The disintegration matrices for the heavy and the light states are denoted by $\Gamma_{H}$ and $\Gamma_{L}$ respectively. Similarly, the time evolution of the $\bar{B}^{0}$,

$$
\left|\bar{B}^{0}(0)\right\rangle=\frac{\left|B_{L}^{0}\right\rangle-\left|B_{H}^{0}\right\rangle}{2 q}
$$

is given by,

$$
\left|\bar{B}^{0}(t)\right\rangle=\frac{p}{q} f_{-}(t)\left|B^{0}(0)\right\rangle+f_{+}(t)\left|\bar{B}^{0}(0)\right\rangle
$$




\section{Coherence of the $\Upsilon(4 \mathrm{~S})$ state}

As the $\Upsilon(4 \mathrm{~S})$ has a total spin of $1 \hbar$, the $\mathrm{B}$ mesons from the $\Upsilon(4 \mathrm{~S})$ decay will be in a coherent $J=1$ state. The bosonic B meson states are also subject to Bose-Einstein statistics. Thus, at any given time there will be exactly one $B^{0}$ and one $\bar{B}^{0}$ each of which will, of course, evolve according to the time dependent Schrödinger equation. Consider now the evolution of the B mesons in the $\Upsilon(4 \mathrm{~S})$ rest frame. Let one of the $\mathrm{B}$ mesons be produced at an angle $\theta$ relative to the beam (z) axis with an angle $\phi$ in the azimuth. Then, the other, must be produced at an angle $\pi-\theta$ to the beam axis with an azimuthal angle of $\phi+\pi$. If $t_{b}$ and $t_{f}$ are the proper times of the backward and forward going $\mathrm{B}$ mesons respectively, the time evolution of the two states in the $\Upsilon(4 \mathrm{~S})$ rest frame is given by,

$S\left(t_{f}, t_{b}\right)=\frac{1}{\sqrt{2}}\left[B^{0}\left(t_{f}, \theta, \phi\right) \bar{B}^{0}\left(t_{b}, \pi-\theta, \pi+\phi\right)-\bar{B}^{0}\left(t_{f}, \theta, \phi\right) B^{0}\left(t_{b}, \pi-\theta, \pi+\phi\right)\right] \sin (\theta)$

Expressing the above in terms of the initial states,

$$
\begin{array}{r}
S\left(t_{f}, t_{b}\right)=\frac{1}{\sqrt{2}} e^{-(\gamma / 2+i \mathcal{M})\left(t_{b}+t_{f}\right)}\left[\frac{1}{2} \cos \left(\Delta m_{d}\left(t_{f}-t_{b}\right)\right)\left(B_{f}^{0} \bar{B}_{b}^{0}-\bar{B}_{f}^{0} B_{b}^{0}\right)\right. \\
\left.-\frac{i}{2} \sin \left(\Delta m_{d}\left(t_{f}-t_{b}\right)\right)\left(\frac{p}{q} B_{f}^{0} \bar{B}_{b}^{0}-\frac{q}{p} B_{b}^{0} \bar{B}_{f}^{0}\right)\right] \sin (\theta)
\end{array}
$$

where, the shorthand $B_{f}^{0}$, etc., have been used to define the initial states,

$$
\begin{aligned}
& B_{f}^{0}=B^{0}\left(t_{f}, \theta, \phi\right) \\
& B_{b}^{0}=B^{0}\left(t_{b}, \pi-\theta, \pi+\phi\right)
\end{aligned}
$$

When $t_{b}=t_{f}$, i.e., when both physical states are present, there is exactly one $B^{0}$ and one $\bar{B}^{0}$. But, if one of the states were to decay, the oscillatory sinusoidal term would be non-zero and the other B would thus mix until its subsequent decay. It should be noted that this coherence nature of the $\Upsilon(4 \mathrm{~S})$ is crucial to the physics reach attainable by the $B A B A R$ experiment. It is this which makes it possible to do the time dependent mixing measurement of $\Delta m_{d}$ using the approach described in chapter 4 . 


\subsubsection{Decays of the B meson states}

In the preceding section, the time evolution of the $\mathrm{B}$ eigenstates were described in terms of the mixed states. Consider now the decay of such a mixed state into a CP eigenstate. Let $\eta_{C P}$ be a $\mathrm{CP}$ eigenstate and $H$ be the Hamiltonian that governs the decay. The amplitude of the decay is then given by,

$$
A=\left\langle\eta_{C P}|\hat{H}| B^{0}\right\rangle
$$

for the $B^{0}$ and

$$
\bar{A}=\left\langle\eta_{C P}|\hat{H}| \bar{B}^{0}\right\rangle
$$

for the $\bar{B}^{0}$. Before continuing, a few words should be said about the phases that may appear in $A$ and $\bar{A}$. Any complex parameters in the Lagrangian that contribute to the amplitudes will appear in a complex conjugate form in the CP conjugate amplitude. Such phases will therefore have opposite signs in the amplitudes, $A$ and $\bar{A}$. In the Standard model, as previously stated, complex phases can only occur in the electroweak part of the theory. Even though these phases, known as "weak phases", are convention dependent, their differences are convention independent. This is a result of the phase rotations in the initial and final terms being the same. It is also possible for scattering/decay amplitudes to contain phases even when the Lagrangian is real. These phases, known as "strong phases", occur in $A$ and $\bar{A}$ with the same sign and do not violate CP. Again, what is of importance is the relative strong phase difference and not the overall phase. Thus, the decay amplitude can written in 3 parts: its magnitude $A_{i}$; the weak phase term $e^{i \phi_{i}}$; and the strong phase term $e^{i \delta_{i}}$. If several amplitudes contribute to $A, A$ can be written as,

$$
A=\Sigma_{i} A_{i} e^{i\left(\delta_{i}+\phi_{i}\right)}
$$

and

$$
\frac{\bar{A}}{A}=\frac{\Sigma_{i} A_{i} e^{i\left(\delta_{i}-\phi_{i}\right)}}{\Sigma_{i} A_{i} e^{i\left(\delta_{i}+\phi_{i}\right)}}
$$

The parameter $\lambda$ can now be defined as, 


$$
\lambda=\frac{q \bar{A}}{p A} .
$$

What is of interest are the time dependent amplitudes that are given by,

$$
\begin{aligned}
\left\langle\eta_{C P}|\hat{H}| B^{0}(t)\right\rangle & =A\left(f_{+}(t)+\lambda f_{-}(t)\right) \\
\left\langle\eta_{C P}|\hat{H}| \bar{B}^{0}(t)\right\rangle & =A \frac{p}{q}\left(f_{-}(t)+f_{+}(t)\right)
\end{aligned}
$$

The decay rate of the $B^{0}$ to a $\mathrm{CP}$ eigenstate is then given by,

$$
\begin{gathered}
\Gamma\left(B^{0}(t) \rightarrow \eta_{C P}\right)=\left|\left\langle\eta_{C P}|\hat{H}| B^{0}(t)\right\rangle\right|^{2} \\
=|A|^{2}\left[e^{-\gamma t} \cos ^{2}\left(\frac{\Delta m_{d} t}{2}\right)+e^{-\gamma t}|\lambda|^{2} \sin ^{2}\left(\frac{\Delta m_{d} t}{2}\right)\right. \\
\left.+e^{-\gamma t} \lambda \cos ^{*}\left(\frac{\Delta m_{d} t}{2}\right) \sin \left(\frac{\Delta m_{d} t}{2}\right)-e^{-\gamma t} \lambda^{*} \cos \left(\frac{\Delta m_{d} t}{2}\right) \sin ^{*}\left(\frac{\Delta m_{d} t}{2}\right)\right] \\
\Gamma\left(B^{0}(t) \rightarrow \eta_{C P}\right)=|A|^{2} e^{-\gamma t}\left[\left(\frac{1+|\lambda|^{2}}{2}\right)+\left(\frac{1+|\lambda|^{2}}{2}\right) \cos \left(\Delta m_{d} t\right)-\mathcal{I} m(\lambda) \sin \left(\Delta m_{d} t\right)\right]
\end{gathered}
$$

Similarly, it can be shown that for the $\bar{B}^{0}$, the decay rate is given by,

$$
\Gamma\left(\bar{B}^{0}(t) \rightarrow \eta_{C P}\right)=|A|^{2} e^{-\gamma t}\left[\left(\frac{1+|\lambda|^{2}}{2}\right)-\left(\frac{1+|\lambda|^{2}}{2}\right) \cos \left(\Delta m_{d} t\right)+\mathcal{I} m(\lambda) \sin \left(\Delta m_{d} t\right)\right]
$$

\subsection{Standard Model calculation of $M_{12}$ and $\Delta m_{d}$}

The aim of this section is to calculate $\Delta m_{d}$ in terms of the SM parameters. In doing this, the mass matrix element $M_{12}$ will be calculated first. As shown previously, $\Delta m_{d}$ has a simple relation to $M_{12}$.

$$
\Delta m_{d}=2\left|M_{12}\right|
$$



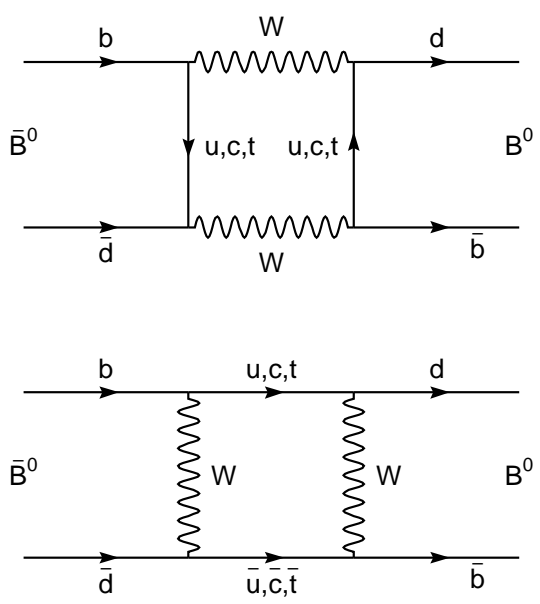

Figure 1.2: Box diagrams for B mixing showing the up, charm, top quark loops. The contribution from the charm and up are negligible compared to that from the top.

The $B^{0} \rightarrow \bar{B}^{0}$ and the $\bar{B}^{0} \rightarrow B^{0}$ mixing transitions are not allowed at tree level in the SM. This is a result of the Bottom quantum number, B, changing by 2 units. Box and higher order diagrams are therefore required to see this effect. The lowest order contribution to $M_{12}$ arises from the box diagrams shown in figure 1.2. $M_{12}$ is in fact roughly proportional to the masses of the internal quark lines. Thus, the box diagrams with internal up or charm quarks are negligible compared to the one with two internal top quark lines. At short distances, $\mathcal{O}\left(\frac{\hbar c}{M_{W}}\right)$, due to the asymptotic freedom property of QCD, the behaviour of the quarks can be expressed in terms of free particles. But, however, at larger distances $\left(\mathcal{O}\left(\frac{\hbar c}{m_{b}}\right)\right) \Delta B=2$ transitions appear as a 4 point fermion interaction, figure 1.3.

For long distance hadronic states, $\mathcal{O}\left(\frac{\hbar c}{\Lambda_{Q C D}}\right)$, QCD effects cannot be described by the exchange of gluons and quarks but have to be described by non-perturbative forces that bind the quarks within the B meson. To correctly include the effects of QCD, the short and long range effects have to be separated. This can be accomplished in a rather elegant way via the Operator Product Expansion (OPE) [22]. The starting point in this study is the effective Hamiltonian. The general effective Hamiltonian can be given by [23],

$$
\mathcal{H}_{e f f}=\frac{G_{F}}{\sqrt{2}} \sum_{i} V_{C K M}^{i} C_{i}(\mu) Q_{i}
$$




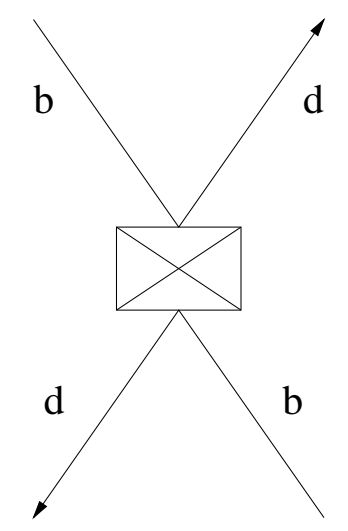

Figure 1.3: The $\Delta B=2$, four quark operator describing the long distance, $\mathcal{O}\left(\frac{\hbar c}{m_{b}}\right)$, effects.

where $V_{C K M}^{i}$ are the elements of the CKM matrix given in equation 1.13. The Wilson coefficients, $C_{i}(\mu)[24]$ are the coupling coefficients associated with the point like operators, $Q_{i}$. The scale, $\mu$, separates the physics contribution to a given decay amplitude into short-distance contributions from scales higher than $\mu$ and longdistance contributions from scales lower than $\mu$. The Wilson coefficients, $C_{i}(\mu)$ are in fact a summary of the physics contributions from scales higher than $\mu$. Due to the asymptotic freedom property of QCD, they can can be calculated using perturbation theory provided that $\mu$ is not too small.

With the above mathematical framework in place, it is now possible to proceed with the calculation of $\Delta m_{d}$. The first step in doing this is to define the effective Hamiltonian that describes the mixing process. The relevant Hamiltonian is the low energy, $|\Delta B=2|$ Hamiltonian, $H^{|\Delta B=2|}$, which can be given as [25],

$$
H^{|\Delta B=2|}=\frac{G_{F}^{2}}{16 \pi^{2}}\left(V_{t b} V_{t d}^{*}\right)^{2} C^{|\Delta B=2|}\left(m_{t}, M_{W}, \mu\right) Q(\mu)+h . c
$$

Here, the hadronic matrix element $\mathrm{Q}$ can be expressed in terms of the $\mathrm{b}$ and $\mathrm{d}$ quarks as,

$$
Q=\bar{d} \gamma_{\nu}\left(1-\gamma_{5}\right) b \bar{d} \gamma^{\nu}\left(1-\gamma_{5}\right) b
$$

As previously stated, the choice of the scale factor, $\mu$ is completely arbitrary. It should be noted that $Q(\mu)$ can only be calculated for $\mu=\mathcal{O}\left(m_{B}\right)$ or less. As as result, the Wilson coefficients, $C^{|\Delta B=2|}\left(m_{t}, M_{W}, \mu\right)$ also have to be calculated for 
this $\mu=\mathcal{O}\left(m_{B}\right)$ scale. The Hamiltonian should not have any scale dependence. As a result, the scale dependence in the operators have to exactly cancel out the scale dependence in the Wilson coefficients. The Wilson coefficients can be calculated at this scale by using the Renormalization Group (RG). The renormalized Wilson coefficients are given by,

$$
C^{|\Delta B=2|}\left(m_{t}, M_{W}, \mu\right)=M_{W}^{2} S\left(\frac{m_{t}^{2}}{M_{W}^{2}}\right) \eta_{B} b_{B}(\mu)
$$

The $\eta_{B}$ and $b_{B}$ coefficients contain the short-distance QCD corrections. To leading order, $\eta_{B}$ and $b_{B}$ are given by,

$$
\begin{aligned}
& \eta_{B}=\left[\alpha_{s}\left(m_{W}\right)\right]^{\frac{6}{23}} \\
& b_{B}=\left[\alpha_{s}\left(m_{W}\right)\right]^{\frac{-6}{23}}
\end{aligned}
$$

The value of $\eta_{B}$ has been calculated using Next to Leading Order (NLO) QCD to yield, $\eta_{B}=0.55[26]$.

The box diagram calculation result, $S(x)$, is given by [25],

$$
S(x)=x\left[\frac{1}{4}+\frac{9}{4} \frac{1}{(1-x)}-\frac{3}{2} \frac{1}{(1-x)^{2}}\right]-\frac{3}{2}\left[\frac{x}{(1-x)}\right]^{3} \ln (x)
$$

In the SM, $M_{12}$ is given by,

$$
M_{12}=\frac{\left\langle B^{0}\left|H^{|\Delta B=2|}\right| \bar{B}^{0}\right\rangle}{2 m_{B}}\left[1+\mathcal{O}\left(\frac{m_{b}^{2}}{M_{W}^{2}}\right)\right]
$$

The hadronic matrix element can be parameterized as,

$$
\left\langle B^{0}|Q(\mu)| \bar{B}^{0}\right\rangle=\frac{8}{3} B_{B}(\mu) f_{B}^{2} m_{B}^{2}=\frac{8}{3} \frac{\hat{B}_{B}}{b_{B}(\mu)} f_{B}^{2} m_{B}^{2}
$$

The mixing parameter, $\Delta m_{d}$, is then given in terms of SM parameters by,

$$
\Delta m_{d}=2\left|M_{12}\right|=\frac{G_{F}^{2}}{6 \pi^{2}} \eta_{B} m_{B} \hat{B}_{B} f_{B}^{2} M_{W}^{2} S\left(\frac{m_{t}^{2}}{M_{W}^{2}}\right)\left|V_{t b} V_{t d}^{*}\right|
$$




\subsection{Experimental status of $\Delta m_{d}$}

The present experimental measurements of $\Delta m_{d}$ are shown in figure 1.4. The present world average without the $B A B A R$ measurements is $0.486 \pm 0.015 \mathrm{ps}^{-1}$ [27].

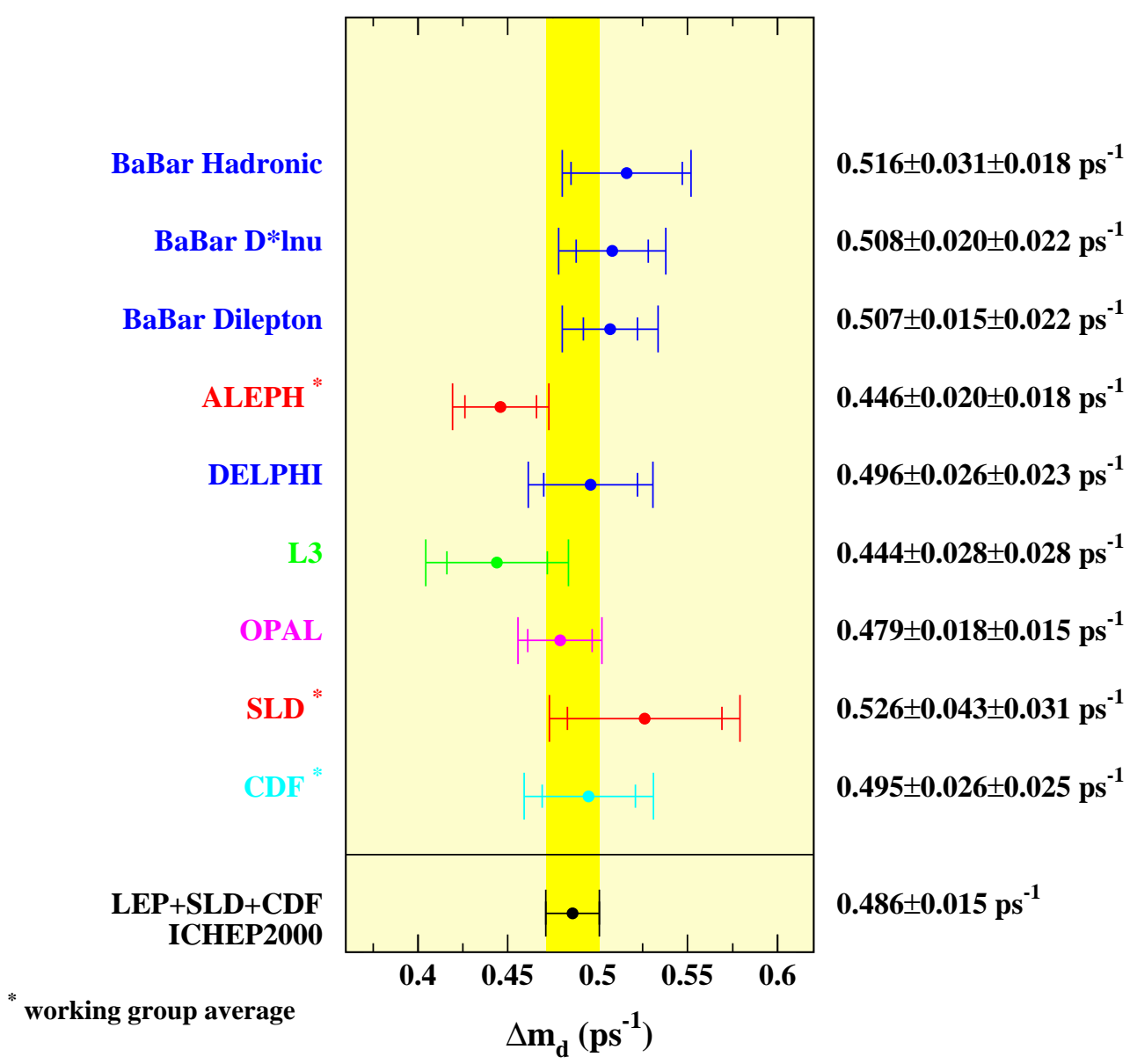

Figure 1.4: The average of the LEP, SLD, CDF and ICHEP $2000 \triangle m_{d}$ results. The BABAR results were not used in the calculation of the average.

As shown above, within the SM framework, mixing occurs via a second order loop diagram involving up/charm/top loops with the contribution from the top being the dominant one. As seen from equation 1.62, $\Delta m_{d}$ is sensitive to the CKM element $\left|V_{t d}\right|$. However, presently, the sensitivity on $\left|V_{t d}\right|$ is not determined by the experimental precision of $\Delta m_{d}$ but by the theoretical uncertainties on the B decay constant, $f_{B}$, and the bag factor, $\hat{B}_{B}$. But, it is expected that the theoretical 


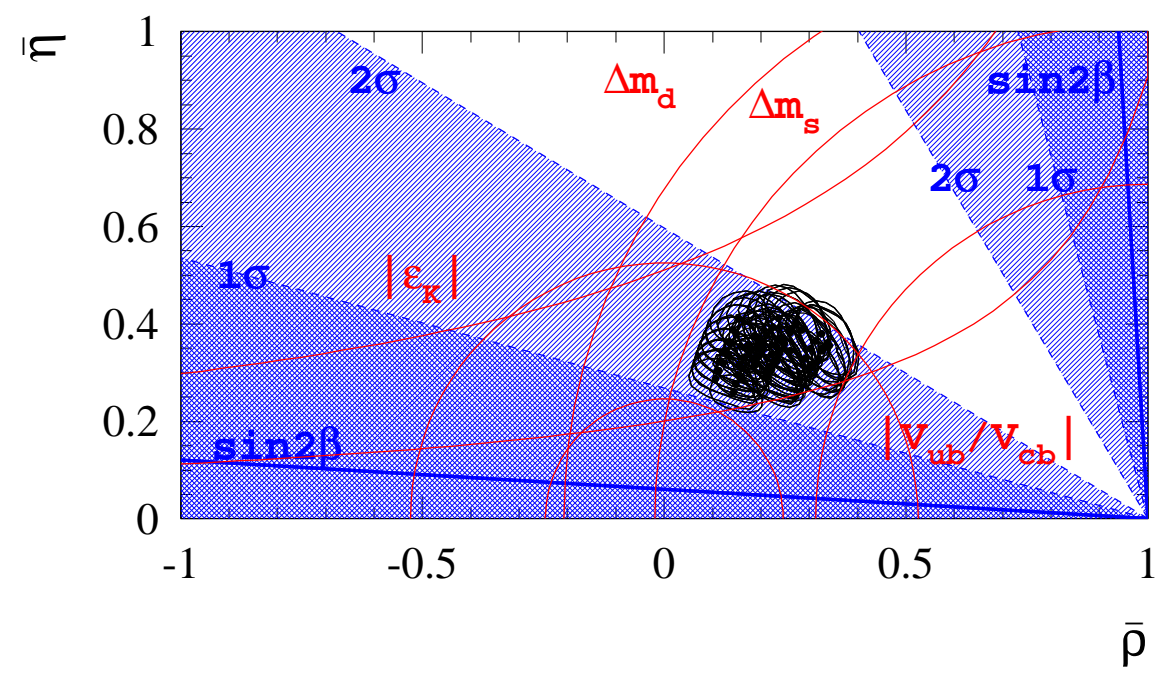

Figure 1.5: A representation of the $(\rho, \eta)$ plane. The results are for the 1999 world average plus the BABAR ICHEP $2000 \sin 2 \beta$ result. The BABAR $\sin 2 \beta$ result is shown by the solid lines with the one and two sigma regions given by the shaded areas. The possible position of the apex of the Unitarity triangle is shown by the marked area.

uncertainties will be reduced. A very high precision measurement of $\Delta m_{d}$ is thus required for a direct comparison with the theoretical prediction once the theoretical uncertainties are comparable with the experimental ones. A representation of the Unitarity triangle with the current experimental results is given in figure 1.5. In addition, an accurate measurement of $\Delta m_{d}$ along with the corresponding measurement from the $B_{S} \bar{B}_{S}$ oscillation $\left(\Delta m_{s}\right)$ can be used to put limits on the Unitarity triangle by using the relation,

$$
\frac{\Delta m_{s}}{\Delta m_{d}}=\frac{m_{B_{s}} \hat{B}_{B_{s}} f_{B_{s}} \eta_{B_{s}}}{m_{B} \hat{B}_{B} f_{B} \eta_{B}} \frac{\left|V_{t b} V_{t s}^{*}\right|}{\left|V_{t b} V_{t d}^{*}\right|}
$$

since the theoretical uncertainties on the ratios of these decay constants and bag factors will be much smaller.

\subsection{Chapter summary}

An introduction into SSB within the SM framework has been given. An overview of the CKM matrix and the Unitarity triangle was presented. A comprehensive description of the $\mathrm{B}$ meson system including the mixing, time evolution and decays 
of the $\mathrm{B}$ mesons was presented. A definition of the mixing parameter $\Delta m_{d}$ was given. It was shown that $\Delta m_{d}$ is proportional to the modulus of the off diagonal mass matrix element, $M_{12}$. The effective Hamiltonian that describes the mixing was presented. Using the $\mathrm{OPE}$ and the RG, $\Delta m_{d}$ was expressed in terms of SM parameters as

$$
\Delta m_{d}=2\left|M_{12}\right|=\frac{G_{F}^{2}}{6 \pi^{2}} \eta_{B} m_{B} \hat{B}_{B} f_{B}^{2} M_{W}^{2} S\left(\frac{m_{t}^{2}}{M_{W}^{2}}\right)\left|V_{t b} V_{t d}^{*}\right|
$$

The current experimental measurement of $\Delta m_{d}$ and the status of the Unitarity triangle using the said measurement were presented. The present world average value of $\Delta m_{d}$ was shown to be $0.486 \pm 0.015 \mathrm{ps}^{-1}$. It was concluded that the overall sensitivity on the CKM element $\left|V_{t d}\right|$ is not limited by experimental precision but by theoretical uncertainties. 


\section{Chapter 2}

\section{The BABAR Detector \& the PEP-II Storage Ring}

\subsection{Introduction}

The BABAR detector, [28] built at the Stanford Linear Accelerator Centre (SLAC), is used to make precise measurements of $\mathrm{CP}$ violation in the $\mathrm{B}$ meson system. To do this, BABAR utilizes the PEP-II [29] asymmetric storage ring. PEP-II collides electrons and positrons stored in two separate rings within the interaction region of the BABAR detector. The asymmetric configuration of PEP-II leads to a relativistic boost of the produced B mesons.

An overview of the BABAR detector and the PEP-II asymmetric ring will be given in this chapter. The chapter will begin with an overview of the PEP-II storage ring. This will be followed by a description of the BABAR detector sub-components.

\section{$2.2 \quad$ PEP-II storage ring}

\subsubsection{Introduction}

An $e^{+} e^{-}$machine has many advantages over a hadronic collider. It is possible to get a very high signal to background ratio with a relatively clean number of events. At the energies operated by PEP-II, a charge multiplicity of about 11 can be expected. The signal to background ratio for PEP-II is given by,

$$
\frac{\sigma_{b \bar{b}}}{\sigma_{T O T}} \approx 0.28
$$


A machine such as PEP-II that is working at the $\Upsilon(4 \mathrm{~S})$ has its own advantages. These include the absence of any fragmentation products and the knowledge of the exact 4-momentum of the B meson system and the magnitudes of the two B meson momenta in the centre of mass system.

\subsubsection{The main storage ring}

The asymmetric PEP-II storage ring at the Stanford Linear Accelerator Center collides $9.0 \mathrm{GeV}$ electrons with $3.1 \mathrm{GeV}$ positrons with a bunch crossing period of $4.2 \mathrm{~ns}$. PEP-II has a design luminosity of $3 \times 10^{33} \mathrm{~cm}^{-2} \mathrm{~s}^{-1}$ with a possible upgrade to $10^{34} \mathrm{~cm}^{-2} \mathrm{~s}^{-1}$. Due to the beam energies being unequal, two rings are required. The electrons are housed in the High Energy Ring (HER) and the positrons in the Low Energy Ring (LER). Both rings are housed in the $2.2 \mathrm{~km}$ long former PEP tunnel. The design of PEP-II calls for 1658 bunches in each ring. The HER design contains $2.1 \times 10^{10}$ electrons per bunch and the LER, $5.9 \times 10^{10}$ positrons per bunch [30]. The collisions give a centre of mass energy of $10.58 \mathrm{GeV}$ which corresponds to the $\Upsilon(4 \mathrm{~S})$ resonance. The $\Upsilon(4 \mathrm{~S})$ has a cross section of $1.05 \mathrm{nb}$ and decays $50 \%$ of the time to $B^{0} \bar{B}^{0}$ pairs, giving a $B^{0} \bar{B}^{0}$ production rate of $1.5 \mathrm{~Hz}$ at the design luminosity. As previously mentioned, the asymmetric design of PEP-II leads to a boost in the $\Upsilon(4 \mathrm{~S})$ rest frame. The magnitude of the boost is given by $\beta \gamma=0.556$. This gives an average separation of $\gamma \beta c \tau=270 \mu \mathrm{m}$ between the two B vertices.

\begin{tabular}{|c|c|}
\hline Parameter & Value \\
\hline \hline$e^{-}$energy & $9.000 \mathrm{GeV}$ \\
$e^{+}$energy & $3.109 \mathrm{GeV}$ \\
CMS energy & $10.58 \mathrm{GeV}$ \\
Beam crossing & $4.2 \mathrm{~ns}$ \\
Boost $(\beta \gamma)$ & 0.556 \\
Mean B separation & $270 \mu \mathrm{m}$ \\
\hline
\end{tabular}

Table 2.1: Relevant parameters for the PEP-II asymmetric collider.

The luminosity delivered by PEP-II can be calculated as,

$$
\mathcal{L}=2.17 \times 10^{34} \xi(1+r) \frac{I E}{\beta_{y}^{*}} \mathrm{~cm}^{-2} \mathrm{~s}^{-1}
$$


where $\beta_{y}^{*}$ is the y component of the betatron function at the Interaction Point (IP), $\xi$ is the beam-beam tune shift, $E$ is the energy of the beam in $\mathrm{GeV}$ and $I$, the current in Amperes. The beam aspect ratio (r), the ratio of the beam height to the beam width at the IP, is given by,

$$
r=\left(\frac{\epsilon_{y} \beta_{y}^{*}}{\epsilon_{x} \beta_{x}^{*}}\right)^{1 / 2}
$$

For PEP-II, an aspect ratio of 0.04 and a beam-beam tune shift of 0.03 were chosen. The RF was chosen to be $2.856 \mathrm{GHz}$ which corresponds to $1 / 16^{\text {th }}$ of the frequency of the SLAC linac.

\subsubsection{The PEP-II interaction region}

The asymmetric design of PEP-II leads to a rather complex Interaction Region (IR). The beams are brought together by a collection of dipole and quadrapole magnets as shown in figure 2.1. Of the magnets utilized to focus the beams, the B1 magnets have the most impact on the acceptance of BABAR.

\subsubsection{The PEP-II beam spot}

The design of the PEP-II collider is set to give a beam spot with transverse dimensions of $15 \mu \mathrm{m}$ in the $\mathrm{y}$ direction and $150 \mu \mathrm{m}$ in the $\mathrm{x}$ direction. It is also expected to be $1 \mathrm{~cm}$ in length along the beam axis ( $\mathrm{z}$ axis). The beam directions are in fact rotated by $20 \mathrm{mr}$ around the y axis. As shall be seen in chapter 5 , the beam spot parameters play an important role at $B A B A R$ where an accurate knowledge of which is crucial to the study of $\mathrm{B}$ meson mixing and $\mathrm{CP}$ asymmetries.

\subsection{The BABAR detector}

The BABAR detector, shown in figure 2.2, was designed to maximally utilize the physics potential of the PEP-II collider ring. It was designed to be an asymmetric detector with the maximum possible acceptance in the centre-of-mass system. This allows $B A B A R$ to take full advantage of the boost provided by PEP-II. It is also designed to be able to accommodate machine components near the IP. Excellent vertex resolution is crucial to the study of $\mathrm{CP}$ violation effects and the measurement 


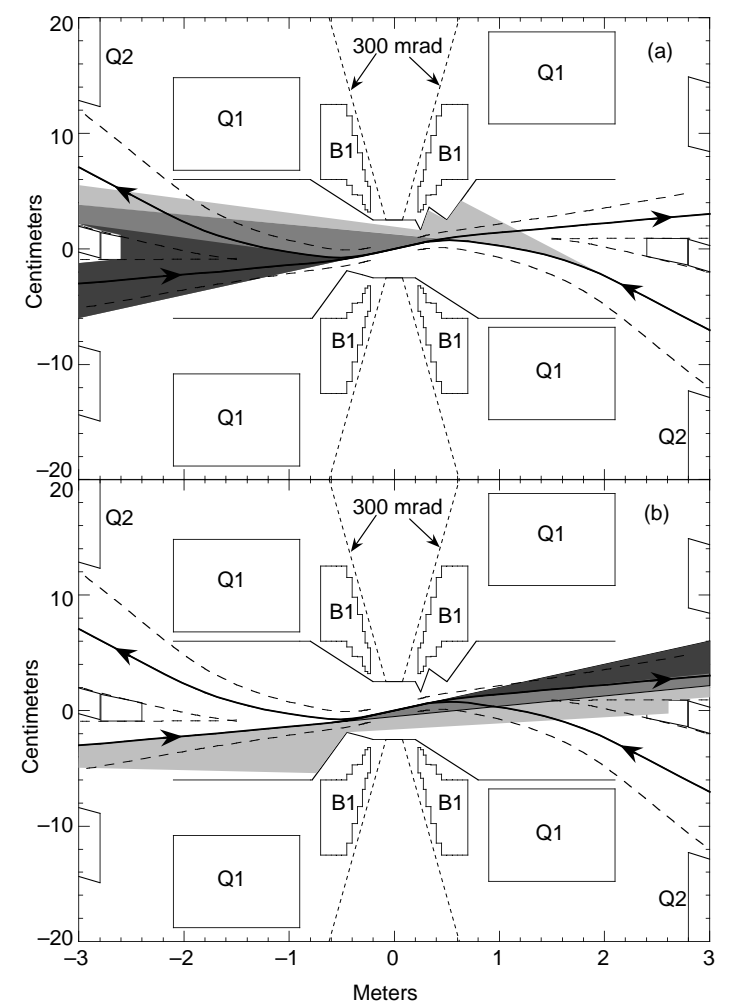

Figure 2.1: The PEP-II interaction region: The synchrotron radiation deposited by the HER (top) and the LER (bottom).

of $\Delta m_{d}$. The BABAR vertex detector was designed to provide the vertex resolution required for the above analyses. $B A B A R$ is designed to provide tracking information for particles with a transverse component of momentum, $p_{\mathrm{t}}$, between $60 \mathrm{MeV} / \mathrm{c}$ and $4 \mathrm{GeV} / \mathrm{c}$. This is to be accomplished by the vertex detector and the drift chamber. Particle identification, in particular the ability to discriminate between $e, \mu, \pi, K$ and $p$, over a wide kinematic range is required to correctly tag the $\mathrm{B}$ mesons. The $B A B A R$ calorimeter was designed with the ability to detect photons in the energy range of $20 \mathrm{MeV}$ to $5 \mathrm{GeV}$. BABAR also contains a superconducting coil that provides a $1.5 \mathrm{~T}$ solenoidal magnetic field. The BABAR detector was commissioned in 1999 and recorded its first event on the $26^{\text {th }}$ of May 1999.

\subsubsection{The BABAR Detector Coordinate System}

$B A B A R$ uses a right handed coordinate system. The $\mathrm{z}$ axis is defined to be pointing along a line that is rotated $20 \mathrm{mr}$ in the plane perpendicular to the high energy 


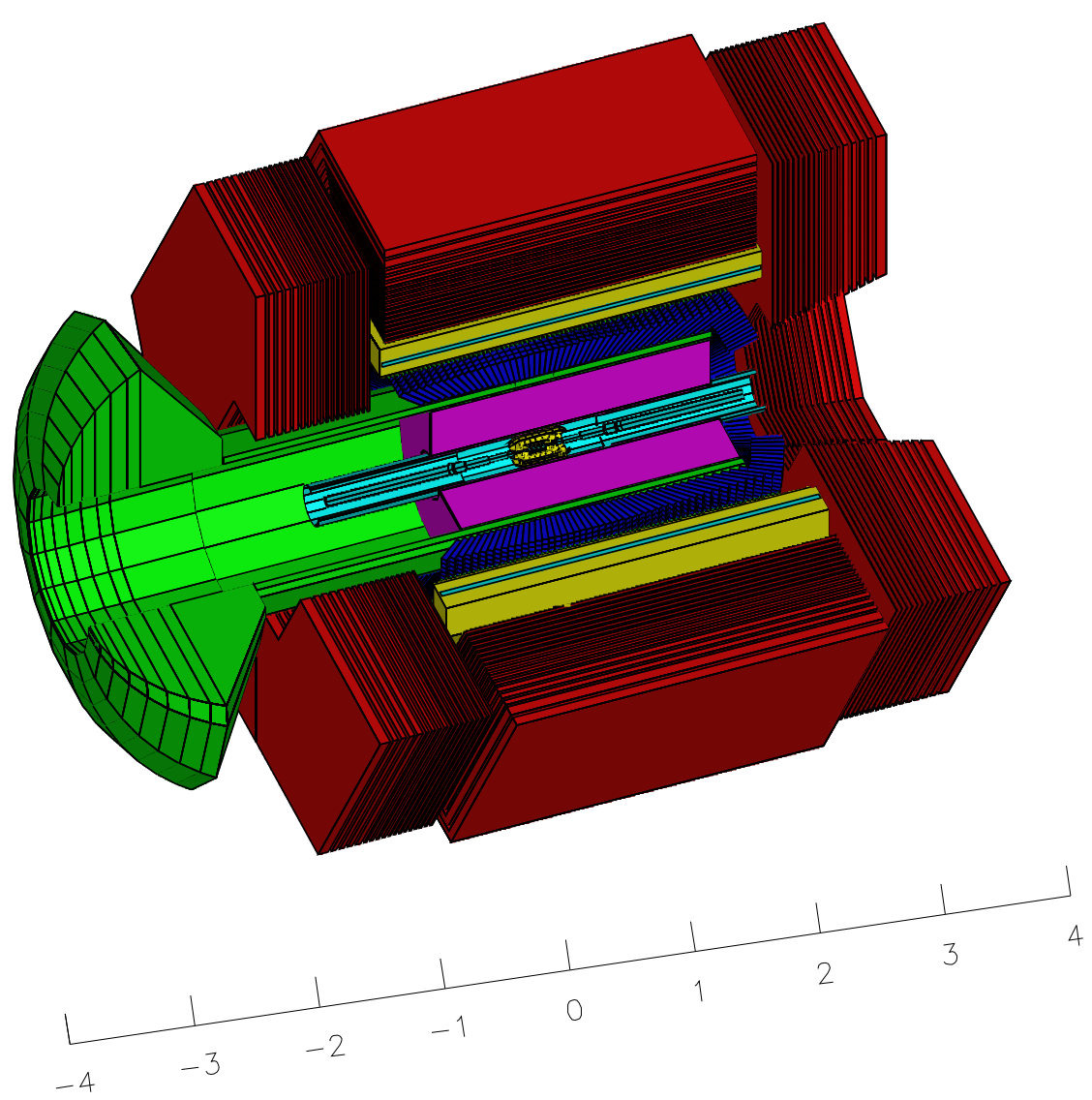

Figure 2.2: The BABAR Detector.

(electron) beam. The y axis is defined to point vertically upwards.

\subsubsection{Silicon Vertex Tracker (SVT)}

\section{SVT physics requirements}

An accurate measurement of $\Delta m_{d}$ and time dependent $\mathrm{CP}$ asymmetries requires the positions of the two B decay vertices to be known to very high precision. These vertices are used to calculate the proper time between the decays. The basic requirement for the silicon detector from a physics point of view is the ability to measure the $\mathrm{z}$ separation between the two vertices to an accuracy of better than $\sim 135 \mu \mathrm{m}$ [31]. This value corresponds to one half the mean separation between the two $\mathrm{B}$ mesons at PEP-II. Thus an accuracy of better than $\sim 80 \mu \mathrm{m}$ is required for each vertex. The $B A B A R$ vertex detector, figure 2.3 , was built with the above criteria in mind. As particles with a momentum of less than $100 \mathrm{MeV} / \mathrm{c}$ do not reach the 
drift chamber, the tracking information for these particles has to come from the silicon detector. The vertex detector thus doubles as a tracking detector for low momentum charged particles. In addition to this, the SVT has to be able to survive the radiation dose delivered by PEP-II. It is expected that the inner detector will be exposed to $240 \mathrm{kr} / \mathrm{yr}$ with the electronics exposed to $110 \mathrm{kr} / \mathrm{yr}$ of radiation.

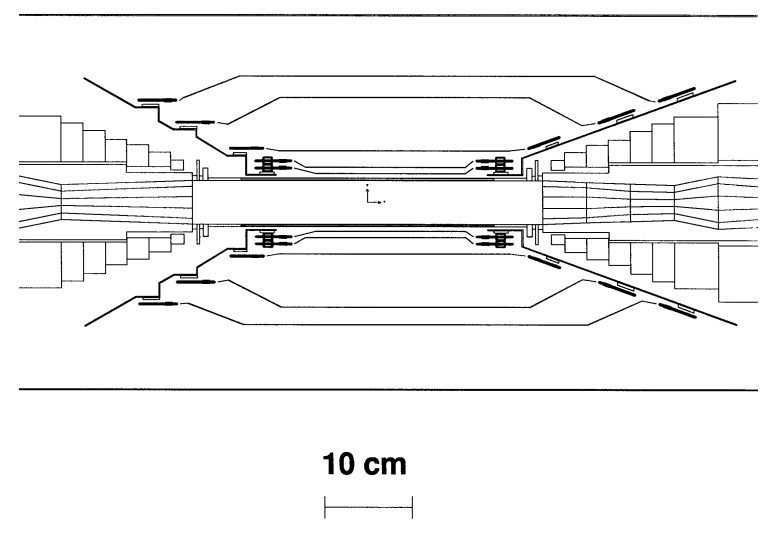

Figure 2.3: The BABAR Silicon Vertex Tracker: A cross sectional view of the SVT in the yz plane. The IP is marked at the centre.

\section{SVT design}

The BABAR SVT consists of 340 silicon detectors that cover an area of about $1 \mathrm{~m}^{2}$ with about 150,000 readout channels. The SVT is made up of double sided silicon detectors in five concentric layers. These layers are further divided into modules in the azimuthal direction. The inner three layers, which have a barrel type structure, consist of six such detector modules. The pin wheel type arrangement of the modules in these layers guarantees overlap between modules within a layer. In order to decrease multiple scattering, the outer two layers are made with an arch like architecture, figure 2.4. These two layers are made up of 16 and 18 detector modules in the azimuth, respectively. In order to increase the overlap within the layers, they are further divided into two modules, "a" and "b", with the "a" type modules being situated at smaller radii than the "b" type ones.

The detector modules are made up of two electrically separate half-modules. Each of these half-modules contain from two to four detectors which are connected by wire bonds. The modules are supported on Kelvar ribs which are mounted on the 
end cones in the forward and backward regions. This entire structure is supported on a carbon fiber support frame which is mounted on the bending magnets.

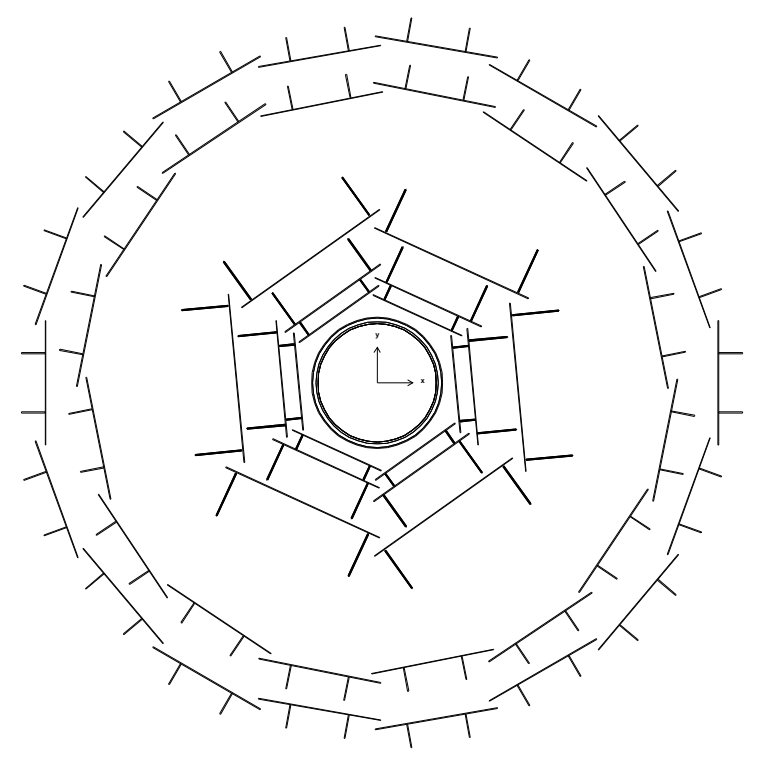

Figure 2.4: The BABAR Silicon Vertex Tracker: A cross sectional view of the SVT in the r $\phi$ plane looking down the beam pipe.

The inner side of the detectors have the silicon strips perpendicular to the beam direction. This side, known as the $\mathrm{z}$-side, is used to measure the $\mathrm{z}$ coordinate. The outer sides ( $\phi$ side) contain longitudinal strips and are used to measure the $\phi$ coordinate. The first three $\mathrm{z}$ layers have a pitch of $100 \mu \mathrm{m}$ while the outer two layers have a pitch of $210 \mu \mathrm{m}$. The first layer has a pitch in $\phi$ of 50 or $100 \mu \mathrm{m}$, the next two layers a pitch of 55 or $110 \mu \mathrm{m}$. The outer two layers have a $\phi$ pitch of $100 \mu \mathrm{m}$. The read-out electronics, which contain silicon micro strip detectors, are placed outside the active area with the $\mathrm{z}$-sides connected to them via Upilex fan out circuits which are glued to the inner faces of the sub-modules. Where the number of strips exceeds the number of read out chains, they are "ganged" or connected together. The $\phi$ strips are daisy chained between the detectors. This results in strips of up to $26 \mathrm{~cm}$ and capacitances of up to $35 \mathrm{pF}$. The silicon micro strip detectors are AC coupled with a polysilicon bias resistors [32] and are fabricated on $(300 \pm 30) \mu \mathrm{m}$ thick, high resistivity silicon. 


\section{SVT electronics and read out system}

The front end signal processing is carried out by Integrated Circuits (ICs) which have been produced via radiation hard CMOS technologies. These are mounted on hybrids [33]. The signals from the strips are amplified, shaped, digitised and compared with a threshold value $[35,36]$. The time interval in which the signal is above the threshold (Time Over Threshold, TOT) is also measured. The logarithm of the TOT is approximately related to the charge induced in the strips. During the latency of the level 1 trigger, the data are stored in front end buffers. On receipt of a level 1 signal from the trigger system, the data are sent via flexible cables to impedance matching cards. The data are then carried by twisted pair cables to multiplexer modules on the detector platform which convert the signals to optical signals. These optical signals are then carried over fibre optic links to the Read Out Modules (ROMs), where the secondary data processing takes place.

\section{SVT performance}

A comparison between data and Monte Carlo (MC) for the first SVT layer resolution as a function of the incident track angle is shown in figure 2.5. The top plot shows the resolution for the first $\mathrm{z}$ layer and the bottom one for the first $\phi$ layer. A resolution of between $15 \mu \mathrm{m}$ to $40 \mu \mathrm{m}$ can thus be obtained in the first z layer.

\subsubsection{Drift Chamber (DCH)}

\section{DCH physics requirements}

The reconstruction of exclusive CP B decay modes requires excellent momentum resolution. This is also required for the study of B meson mixing where the lepton momenta are required when using leptons as tagging particles (section 4.7.1). The BABAR DCH, which provides tracking information for particles with momenta greater than $100 \mathrm{MeV} / \mathrm{c}$, is used for this. The aim of the $\mathrm{DCH}$ is to provide a spatial resolution of better than $140 \mu \mathrm{m}$ in the $r \phi$ plane. It is also expected to provide particle identification using $\mathrm{d} E / \mathrm{d} x$ measurements to a resolution of about $7 \%$ for low momentum tracks and a momentum resolution of $0.3 \% \times p_{\mathrm{t}}$ for tracks with a momentum greater than $1 \mathrm{GeV} / \mathrm{c}$. The design also has to take into account the high occupancies due to synchrotron radiation. 

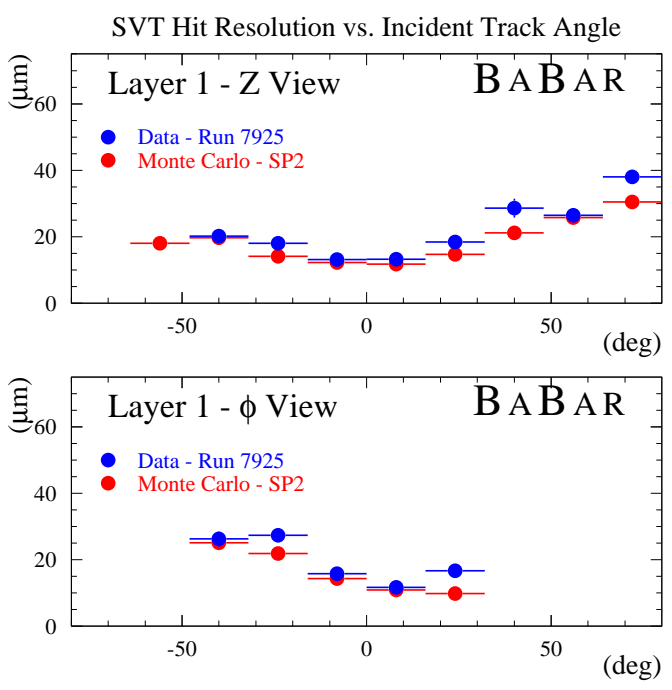

Figure 2.5: The BABAR Silicon Vertex Tracker: Performance plot showing the position resolution in $z$ (top plot) and $\phi$ (bottom plot) for the 1st layer for muon pair events. A z resolution of between $15-40 \mu \mathrm{m}$ is obtained.

\section{DCH design}

A detailed description of the BABAR DCH can be found elsewhere [34]. The DCH is a $280 \mathrm{~cm}$ long cylinder with an inner radius of $23.6 \mathrm{~cm}$ and an outer one of $80.9 \mathrm{~cm}$. As the resolution of low momentum tracks is dominated by multiple scattering, the inner cylinder has been built in such a way as to minimise these effects. The outer cylinder is made up of two carbon fibre layers on a Normex core that corresponds to $1.5 \%$ radiation lengths $\left(X_{0}\right)$. The inner cylinder in made of $1 \mathrm{~mm}$ beryllium. Due to the boost in the forward (electron) direction of $B A B A R$, the aluminum end plates of the chamber are of different thicknesses. The forward end plate is $15 \mathrm{~mm}$ thick whilst the backward one has a thickness of $24 \mathrm{~mm}$.

The chamber itself is made up of ten axial (A) and stereo $(\mathrm{U}, \mathrm{V})$ superlayers of 7104 hexagonal drift cells, each with a typical dimension of $1.2 \times 1.8 \mathrm{~cm}^{2}$. The superlayers in turn, are made up of four more layers and are set up in a AUVAUVAUVA configuration, figure 2.6. For the stereo superlayers, the stereo angle varies from $40 \mathrm{mr}$ to $70 \mathrm{mr}$ in the outermost layer. The chamber is filled with a helium-isobutane (80\% : 20\%) gas mixture. The field wires are $120 \mu \mathrm{m}$ and $80 \mu \mathrm{m}$ gold-plated aluminium and are subject to a nominal voltage of $340 \mathrm{~V}$ from high voltage assemblies 


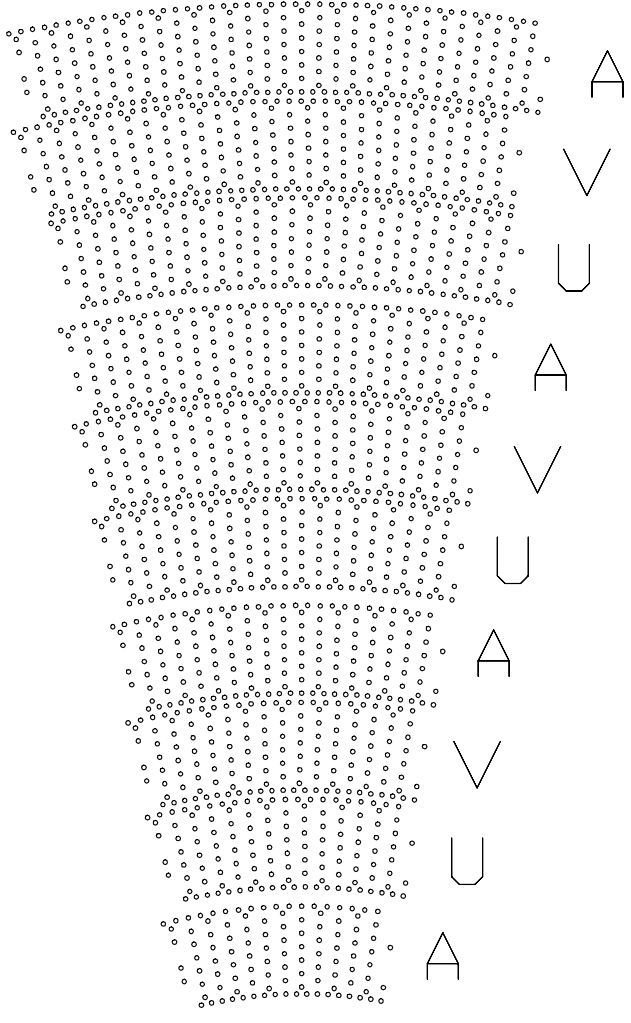

Figure 2.6: The BABAR Drift Chamber: The Axial $(A)$ and Stereo $(U, V)$ superlayers containing the hexagonal drift cells.

at the rear endplate. The sense wires are $20 \mu \mathrm{m}$ gold plated tungsten-rhenium and are subject to a potential of $1900-1960 \mathrm{~V}$ at the boundaries of the superlayers.

\section{DCH electronics and read out system}

In order to measure the drift time, the leading edge of the signal from the charge arriving at the sense wire is detected and digitised. This is done to a 1 ns resolution. Summing the total charge in this pulse then gives the $\mathrm{d} E / \mathrm{d} x$. In doing this, a slow shaper is applied and the resulting pulse is digitised with a six-bit $15 \mathrm{MHz}$ FADC. A four channel amplifier IC and an eight channel CMOS TDC/FADC IC [37] are used. The amplifiers, digitisers and the trigger interface electronics are contained in 48 wedge shaped aluminum boxes that are mounted on the rear endplate. The data from the TDCs and FADCs are written into four levels of event buffers through a $12 \mu$ s trigger latency buffer. 


\section{DCH performance}

The measured $\mathrm{d} E / \mathrm{d} x$ for various track species as a function of their momenta are given in figure 2.7. The $p_{\mathrm{t}}$ resolution for data muon pair events is shown in figure 2.8. The results are in agreement with the design resolution of $0.3 \% \times p_{\mathrm{t}}$.

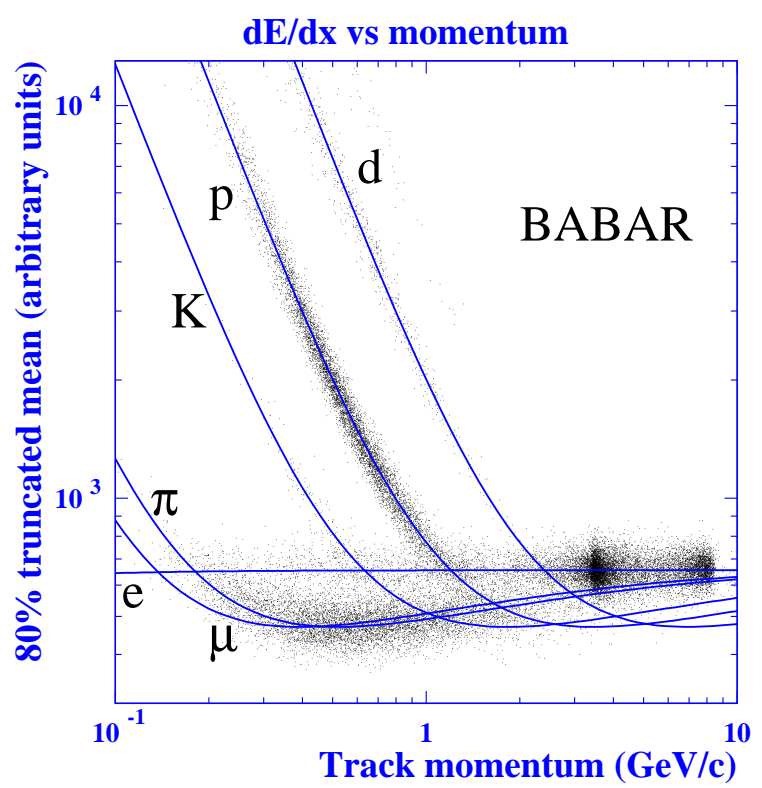

Figure 2.7: The BABAR Drift Chamber: Performance plot showing the $d E / d x$ measurements for electrons, muons, pions, kaons, protons from beam scan data as a function of the track momentum. The data are parameterised using Bethe-Bloch distributions. The protons and deuterons are from beam-gas interactions. Pions are from both beam-gas interactions and hadronic decays. High momentum muons are from cosmics.

\subsubsection{Detector of Internally Reflected Čerenkov Radiation (DIRC)}

\section{DIRC physics requirements}

Excellent particle identification is required to do a systematic study of CP violation and B physics. In particular, excellent pion/kaon separation is required not only for kaon tagging but also for the study of rare B decay modes such as $B^{0} \rightarrow \pi^{+} \pi^{-}$and $B^{0} \rightarrow K^{ \pm} \pi^{\mp}$. This is to be accomplished by the Detector of Internally Reflected Čerenkov Radiation (DIRC). The DIRC accomplishes this by using the Cerenkov radiation emitted by charged particle species. The primary purpose of the DIRC is to provide pion-kaon separation for momenta of up to $4.0 \mathrm{GeV} / \mathrm{c}$. It is also used for muon identification for muons with momenta less than $750 \mathrm{MeV} / \mathrm{c}$. 


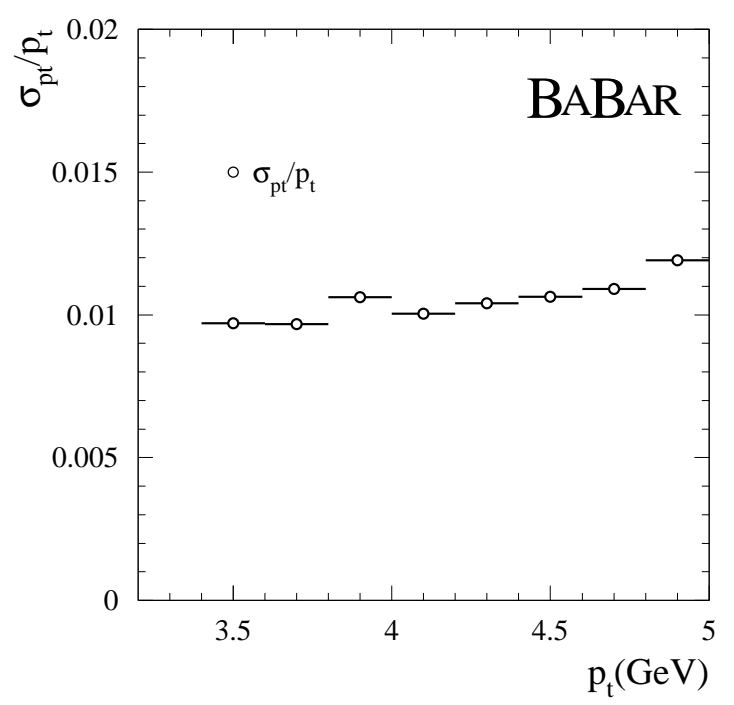

Figure 2.8: The BABAR Drift Chamber: Performance plot showing the $p_{\mathrm{t}}$ resolution for data muon pair events. The results are in agreement with the design resolution of $0.3 \% \times p_{\mathrm{t}}$.

\section{DIRC design}

The DIRC radiator is made up of 144 synthetic fused silica quartz bars which are $1.7 \mathrm{~cm}$ thick, $3.5 \mathrm{~cm}$ wide and $4.9 \mathrm{~m}$ in length, figure 2.9 . The quartz bars have a refractive index of 1.474. Čerenkov radiation produced in the quartz bars are sent to the backward end of the detector by means of total internal reflections, figure 2.10. This insures that the angle of production of the photon is preserved. Photons that are produced in the forward direction are reflected by mirrors and are sent to the back via the aforementioned method. At the backward end of the detector, the Čerenkov image is expanded in $6 \mathrm{~m}^{3}$ of purified water that has a reflective index comparable with that of quartz. This is done to reduce the distortion due to refraction at the quartz-water border. The amount of material present in the DIRC amounts to about $14 \%$ of a radiation length $\left(X_{0}\right)$ for a particle of normal incidence. Radially, it occupies only $8 \mathrm{~cm}$ of space. The DIRC quartz bars extend $187 \mathrm{~cm}$ from the IP and cover $87 \%$ of the solid polar angle in the $\Upsilon(4 \mathrm{~S})$ centre of mass frame. The 144 quartz bars are arranged in 12 modules ("bar boxes"). Due to gaps between the bars at the 12 sides of the polygon, the azimuthal coverage is limited to $93 \%$. The Čerenkov light is detected by an array of 10752 PhotoMultiplier Tubes (PMTs) each with a diameter of $2.82 \mathrm{~cm}$. The PMTs operate at a voltage of $1.1 \mathrm{kV}$ with a maximum 


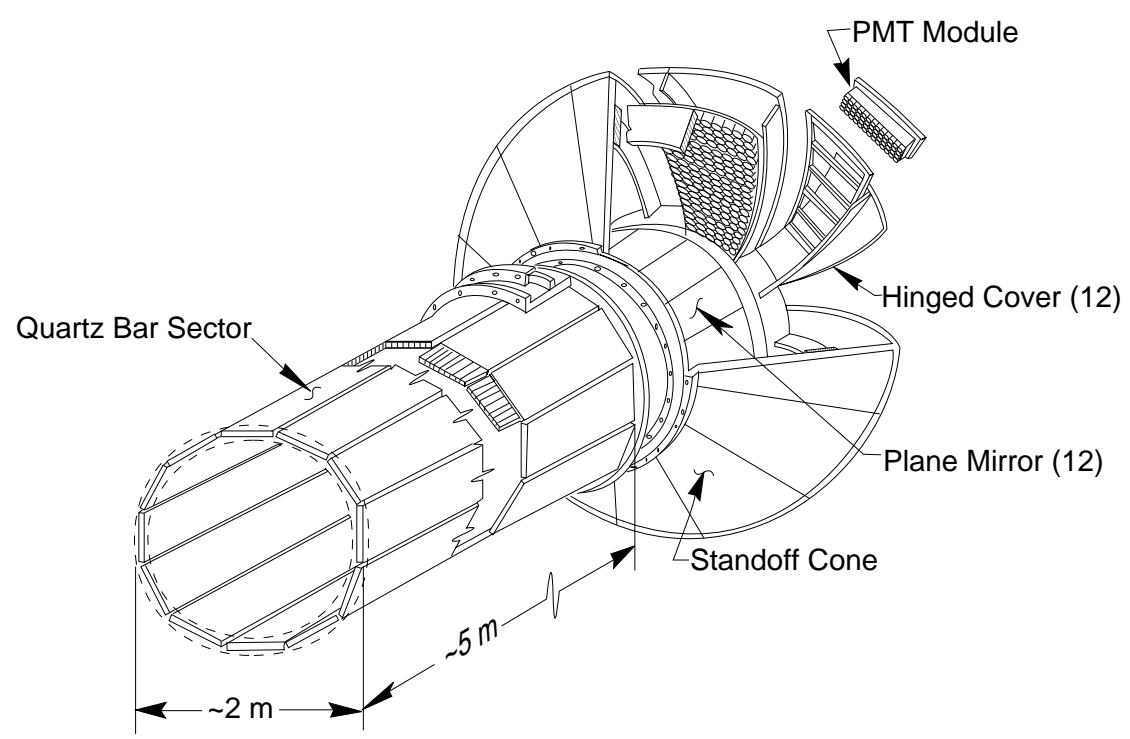

Figure 2.9: The BABAR Detector of Internally Reflected Cerenkov Radiation: A schematic of the DIRC, depicting the quartz radiators and the stand off box.

current per PMT of about $150 \mu \mathrm{A}$. The voltage is provided by a CAEN SY 527 high voltage system. The PMTs operate directly in the water and are situated about $1.2 \mathrm{~m}$ away from the end of the quartz bars. They detect Cerenkov radiation in the visible and near-UV spectrum. The PMTs and the water are contained in an enclosure known as the Stand Off Box (SOB) which is magnetically shielded to provide a suitable working environment for the PMTs. The SOB extends through the steel of the flux return in the backward end.

\section{DIRC electronics and read out}

The data from the PMTs are gathered by 168 DIRC Front End Boards (DFB) with each processing 64 PMT inputs. Each DFB is made of eight analogue chips, four TDC chips and one 8-bit flash ADC (FADC). The signals from the PMTs are first amplified and then shaped by a 8-channel analogue chip. The pulses thus shaped are sent via 1.2 Gbits/s optical fibres to the DIRC ROMs. The Front End Electronics (FEE) system has been designed to measure the arrival time of Čerenkov photons to an accuracy of $1.5 \mathrm{~ns}$. 


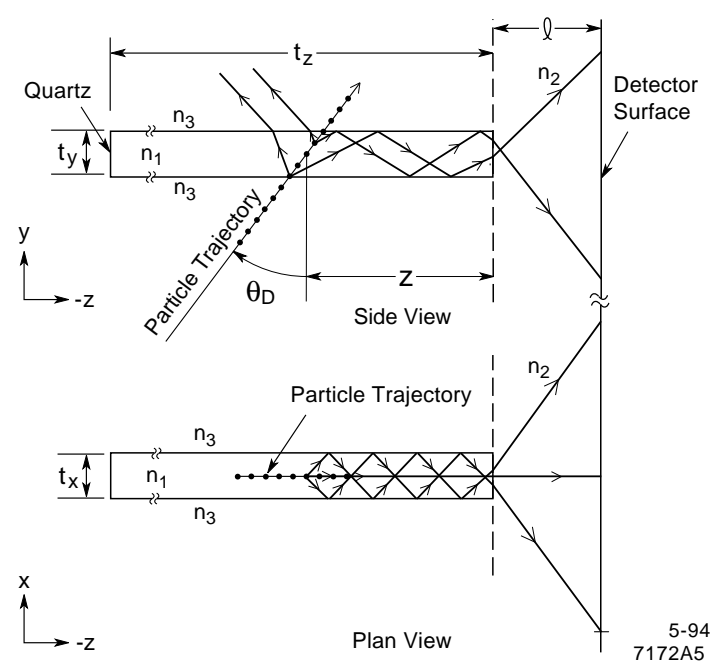

Figure 2.10: The BABAR Detector of Internally Reflected Cerenkov Radiation: The Čerenkov radiation produced by charged particles traversing the quartz are total internally reflected to the detector surface. In doing this, the production angle of the radiation is preserved.

\section{DIRC performance}

In order to get the desired pion-kaon separation, the design calls for a Čerenkov angle resolution per track of $2 \mathrm{mr}$ or better. It is possible to get the required resolution by combining a large number of photoelectrons from an observed track. The single photon Čerenkov angle resolution is shown in figure 2.11. Here, the data are fitted using a double Gaussian distribution where the sigma of the narrow Gaussian was found to be $10.2 \mathrm{mr}$. With an average of about 30 photons per track, the Čerenkov angle resolution per track is about $2.8 \mathrm{mr}$. This corresponds to a three sigma pion kaon separation at $3 \mathrm{GeV} / \mathrm{c}$. At low momenta (less than $750 \mathrm{MeV} / \mathrm{c}$ ), the Čerenkov angle accuracy is dominated by the uncertainty in the track direction arising from multiple scattering in the drift chamber, the DIRC supports and the quartz bars.

\subsubsection{Electromagnetic Calorimeter (EMC)}

\section{EMC physics requirements}

As a large number of the B decay products contain a $\pi^{0}$ which decay $99.9 \%$ of the time into two photons, a calorimeter with very good energy resolution is required. The BABAR calorimeter was designed to have an energy resolution given by, 


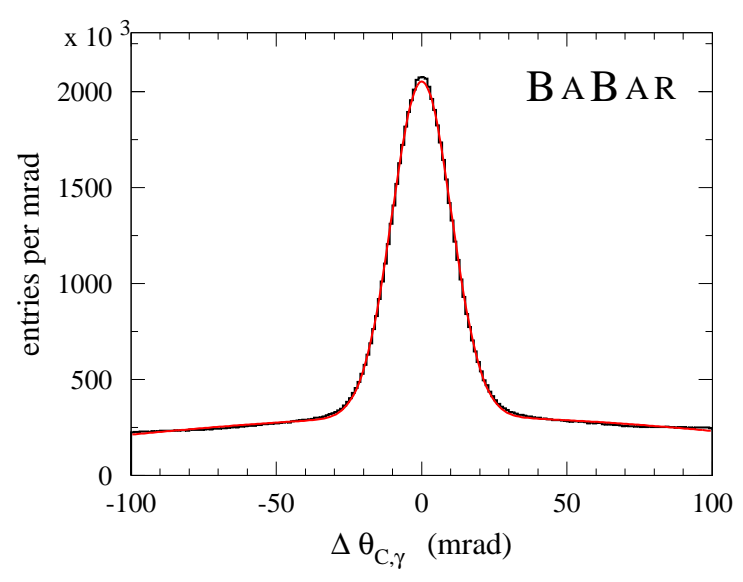

Figure 2.11: The BABAR Detector of Internally Reflected Cerenkov Radiation: The single photon, Cerenkov angle resolution is shown. The data are fitted using a double Gaussian distribution with the $\sigma_{\text {narrow }}=10.2 \mathrm{mr}$.

$$
\frac{\sigma_{E}}{E}=\frac{\sigma_{1}}{\sqrt[4]{E}} \oplus \sigma_{2} \%
$$

with $\sigma_{1} \approx 1 \%$ and $\sigma_{2} \approx 1.2 \%$, for photons incident at a polar angle of $90^{\circ}$. The constant term is due to the front and rear leakage from the crystals $(\leq 0.5 \%)$, inter-calibration errors $(\leq 0.25 \%)$ and non-uniformity of the light collection $(\leq 0.5$ $\%)$. Noise due to electronics and beam background are not included in the above expression. The minimum detectable energy for photons is in the range of 10$20 \mathrm{MeV}$.

The angular resolution for photons at a polar angle of $90^{\circ}$ is given by,

$$
\sigma_{\theta, \phi}=\frac{3 m r}{\sqrt{E(G e V)}}+2 m r
$$

This is dependent on the transverse crystal size and the mean distance from the crystal to the interaction point.

\section{EMC design}

The BABAR EMC is made up of 6580 quasi-projective Thallium-doped Caesium Iodide (CsI) crystals (table 2.2) that cover a solid angle of $-0.78 \leq \cos (\theta) \leq 0.96$ in the laboratory frame. The front faces of the crystals have a typical dimension of about $5 \mathrm{~cm}$. 


\begin{tabular}{|c|c|}
\hline Properties & CsI $(\mathrm{Tl})$ \\
\hline \hline Radiation Length $(\mathrm{cm})$ & 1.85 \\
Moliere Radius $(\mathrm{cm})$ & 3.6 \\
Density $\left(\mathrm{g} / \mathrm{cm}^{3}\right)$ & 41.7 \\
Absorption Length for $5 \mathrm{GeV}$ pions $(\mathrm{cm})$ & 4.53 \\
$d E / d x \|_{m i p}(\mathrm{MeV} / \mathrm{cm})$ & 5.6 \\
Light Yield (photons $\left./ \mathrm{MeV} x 10^{3}\right)$ & $40-50$ \\
Light Yield Temperature Coefficient $\left(\% /{ }^{\circ} C\right)$ & 0.1 \\
Peak Emission $(\mathrm{nm})$ & 565 \\
Refractive Index at Emission Maximum & 1.79 \\
Decay Time $(\mathrm{ns})$ & 940 \\
Radiation Hardness $(\mathrm{rad})$ & $10^{3}-10^{4}$ \\
\hline
\end{tabular}

Table 2.2: Properties of Thallium doped Caesium Iodide, $\mathrm{CsI}(\mathrm{Tl})$, used by the BABAR EMC.

The EMC consists of a cylindrical barrel and a forward end cap, figure 2.12. The barrel weighs 23.5 metric tones and has an inner radius of $90 \mathrm{~cm}$ and an outer one of $136 \mathrm{~cm}$. It is supported at each end by the magnetic coil cryostat and is located between the DIRC and the cryostat. The barrel consists of $5760 \mathrm{CsI}(\mathrm{Tl})$ crystals which are grouped in 280 carbon fibre composite (CFC) modules. Each module, apart from the most backward one, contains seven crystals in the polar $(\theta)$ direction and three in the azimuth $(\phi)$. These modules are mounted on an aluminium strongback which is connected to the coil cryostat. The crystals are thus arranged in 48 rows in $\theta$ with each having 120 crystals in $\phi$. The barrel crystals cover a solid angle of $\pi-684 \mathrm{mr} \leq \theta \leq 470 \mathrm{mr}(-0.775 \leq \cos (\theta) \leq 0.892)$ in the laboratory frame and $-0.916 \leq \cos (\theta) \leq 0.715$ in the $\Upsilon(4 \mathrm{~S})$ centre of mass system.

The forward endcap weighs about 3.2 metric tonnes and is a conic section with the front and back surfaces tilted at $22.7^{0}$ to the vertical. The end cap consists of $820 \mathrm{CsI}(\mathrm{Tl})$ crystals, covering a solid angle range of $468 \mathrm{mr} \leq \theta \leq 275 \mathrm{mr}(0.893 \leq$ $\cos (\theta) \leq 0.962)$ in the laboratory frame and $0.718 \leq \cos (\theta) \leq 0.895$ in the $\Upsilon(4 \mathrm{~S})$ centre of mass frame. The crystals are contained in eight rings in $\theta$ with the inner ring placed at a radius of $55.3 \mathrm{~cm}$ from the beam axis. The outermost three rings contain 120 crystals each and the inner layers contain 100 and 80 crystals. The end cap contains 20 modules in the azimuth with each containing 41 crystals. Just as in the barrel, the modules are made of CFC and are mounted on an aluminium backplate. 


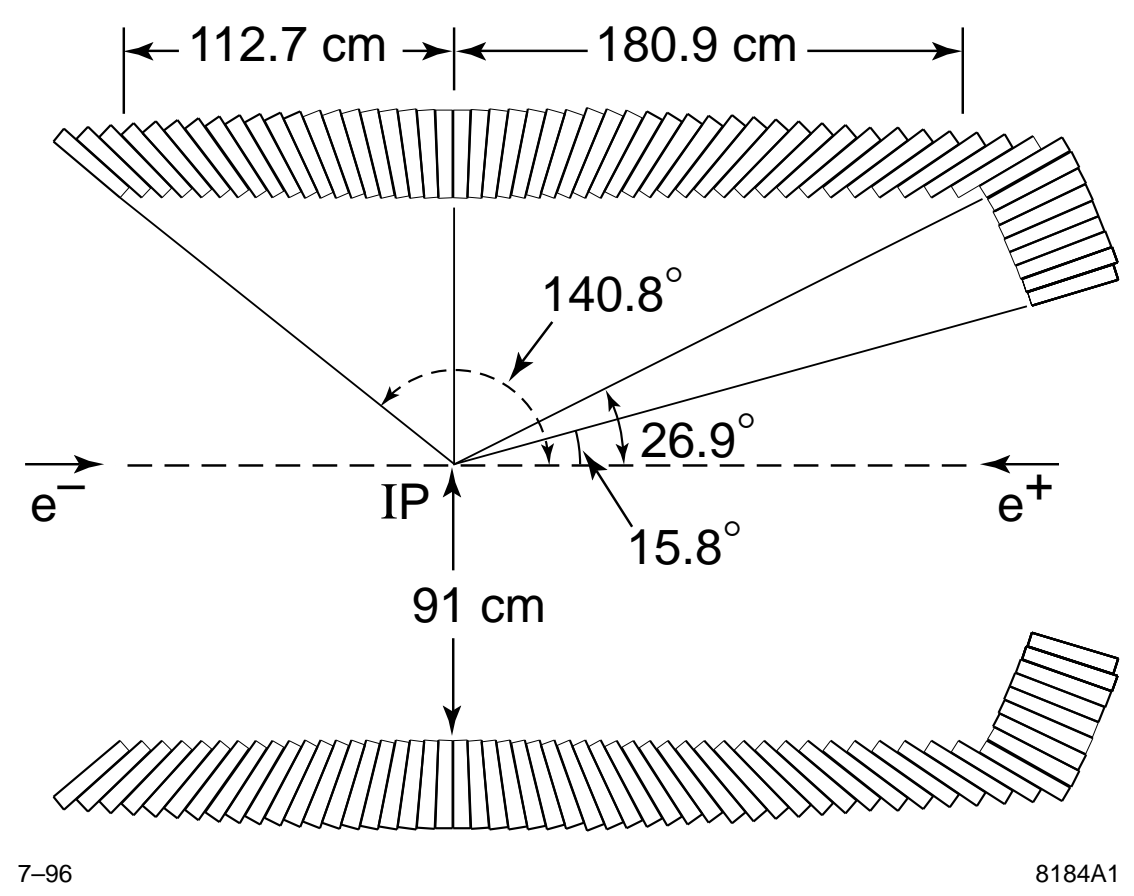

Figure 2.12: The BABAR Electromagnetic Calorimeter: A cross sectional view of the barrel and forward endcap EMC.

The EMC was designed in a way to minimize the material in front of the crystals. The amount of material in front of the barrel at a polar angle of $90^{\circ}$ is about $0.25 X_{0}$. The forward and backward ends of the barrel contain $0.5 X_{0}$ and $0.39 X_{0}$ of material respectively. For the endcap, the amount of external material at a polar angle of $20^{\circ}$ is $0.20 X_{0}$. In each of the $\theta$ and $\phi$ directions, there is a gap of about $1.25 \mathrm{~mm}$ between the crystals as a result of the crystal wrappings and the CFC.

\section{EMC electronics and read out system}

A full discussion of this can be found in chapter 3 .

\section{EMC performance}

The energy resolution for the EMC is shown in figure 2.13. The data are fitted using the distribution given in equation 2.4. From the fit, it can be seen that a value of $\sigma_{1}$ of $(2.32 \pm 0.003 \pm 0.3) \%$ is obtained whereas the design calls for a $\sigma_{1}$ of about $1 \%$. The value of $\sigma_{2}$ was found to be $(1.85 \pm 0.07 \pm 0.1) \%$ whilst the design requirement was $1.2 \%$. The position resolution, $\frac{\sigma(\theta)}{E}$, is shown in figure 2.14 . 


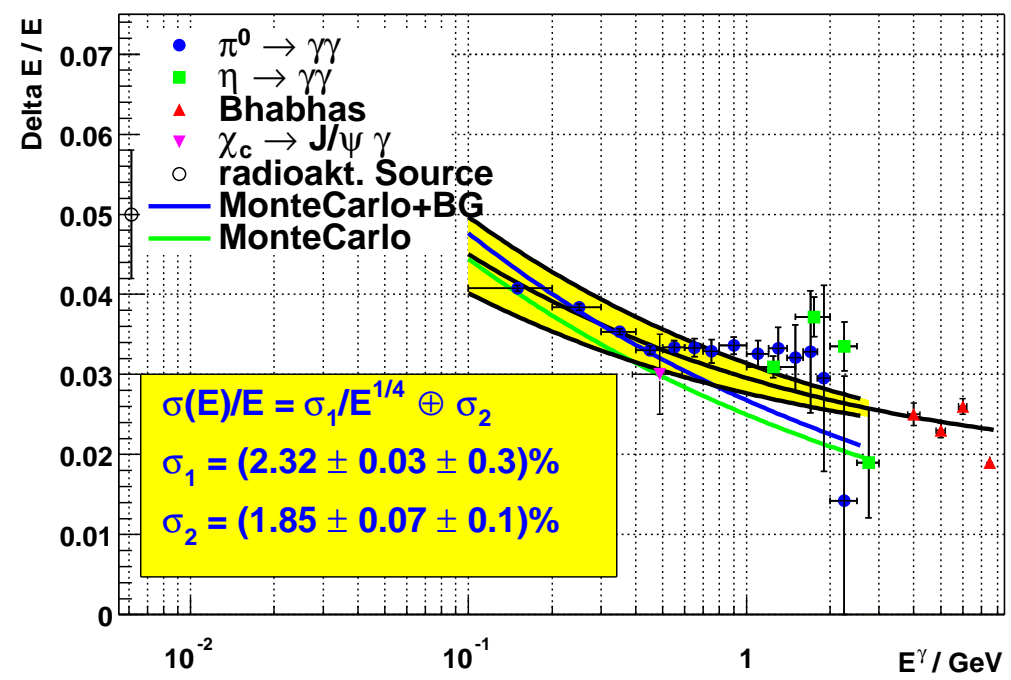

Figure 2.13: The BABAR Electromagnetic Calorimeter: Plot showing the energy resolution. The data points are fit using the distribution given in equation 2.4. The data are from $\pi^{0}$ and $\eta$ to two photon decays, Bhabha events and $\chi_{c} \rightarrow J / \psi \gamma$ events.

\subsubsection{Instrumented Flux Return (IFR)}

\section{IFR physics requirements}

The correct identification of muons is required for the study of many B meson decays. In particular the study of the "Golden Mode" $B^{0} \rightarrow J / \psi K_{S}^{0}$ requires the correct identification and reconstruction of the muons from the decay of the $J / \psi$. Also, due to the large semileptonic branching ratio of $\mathrm{B}$ mesons ( $\approx 10 \%$ to muons), muon identification is crucial to B mixing and exclusive and inclusive semileptonic $\mathrm{B}$ decay studies. This is to be primarily achieved by the BABAR Instrumented Flux Return (IFR). The IFR, along with the calorimeter, also provides neutral particle (e.g. $\left.K_{L}^{0}\right)$ identification.

\section{IFR design}

The IFR consists of a central barrel part and forward and backward endcaps made out of 18 plates of segmented iron. This provides a solid angle coverage down to $300 \mathrm{mr}$ in the forward direction and $400 \mathrm{mr}$ in the backward direction. The innermost nine segmented plates are $2 \mathrm{~cm}$ thick. The next four are $3 \mathrm{~cm}$ thick and these are followed by three more plates which are $10 \mathrm{~cm}$ thick each in the barrel and one of 


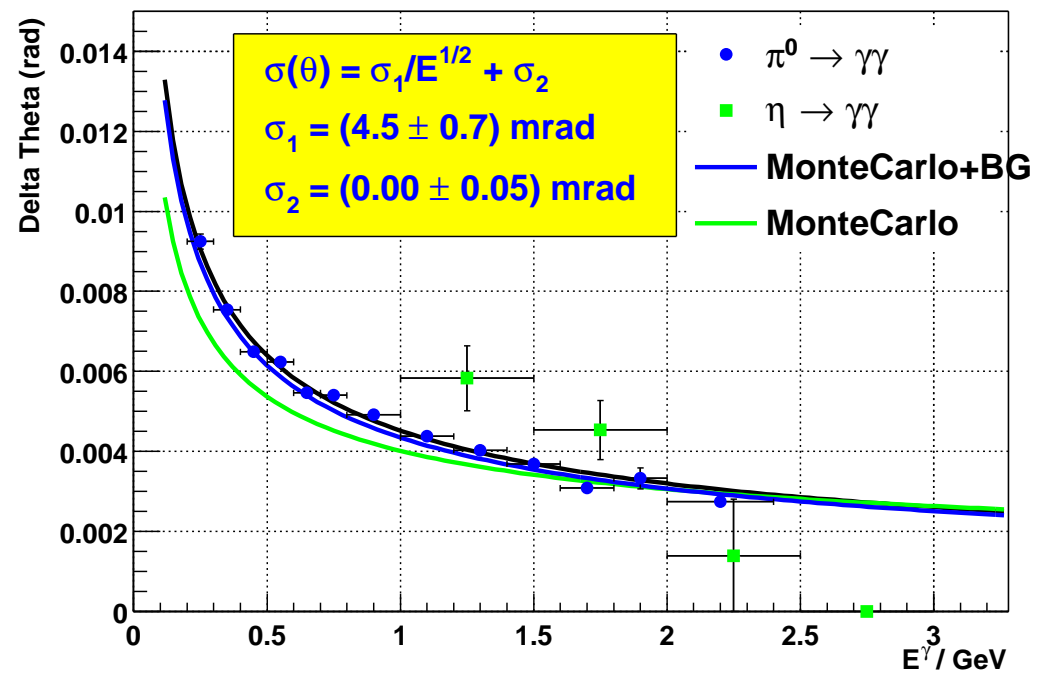

Figure 2.14: The BABAR Electromagnetic Calorimeter: Plot showing the position resolution for $\pi^{0}$ and $\eta$ decays to two photons.

$10 \mathrm{~cm}$ and another two of $5 \mathrm{~cm}$ thickness in the endcaps. This gives a total thickness of $60 \mathrm{~cm}$ in the barrel and $50 \mathrm{~cm}$ in the endcaps. The total area covered by the IFR is over $1000 \mathrm{~m}^{2}$.

The barrel consists of 21 active detector Resistive Plate Counter (RPC) layers, figure 2.15. There is a double layer of cylindrical RPCs surrounding the EMC, an inner layer of planer RPCs which are between the solenoidal coil and the iron, 17 layers of RPCs in the iron gaps and another layer outside the iron structure. The end cap doors are split into two vertical sections so that access to the inner detectors is possible.

The active volume within the IFR is filled with a $56.7 \%$ argon, $38.8 \%$ freon- $134 \mathrm{a}$ and $4.5 \%$ isobutane gas mixture. This mixture was driven by the need to operate the RPCs in a non-flammable, safe environment. The IFR is water cooled to keep the temperature in the barrel at about $20^{\circ} \mathrm{C}$ and the endcaps at about $22-24^{\circ} \mathrm{C}$.

\section{IFR electronics and read out system}

Both sides of the RPCs in the IFR are read out by strip electrodes that run along the chamber both lengthwise and crosswise. This setup provides information in all three dimensions. 


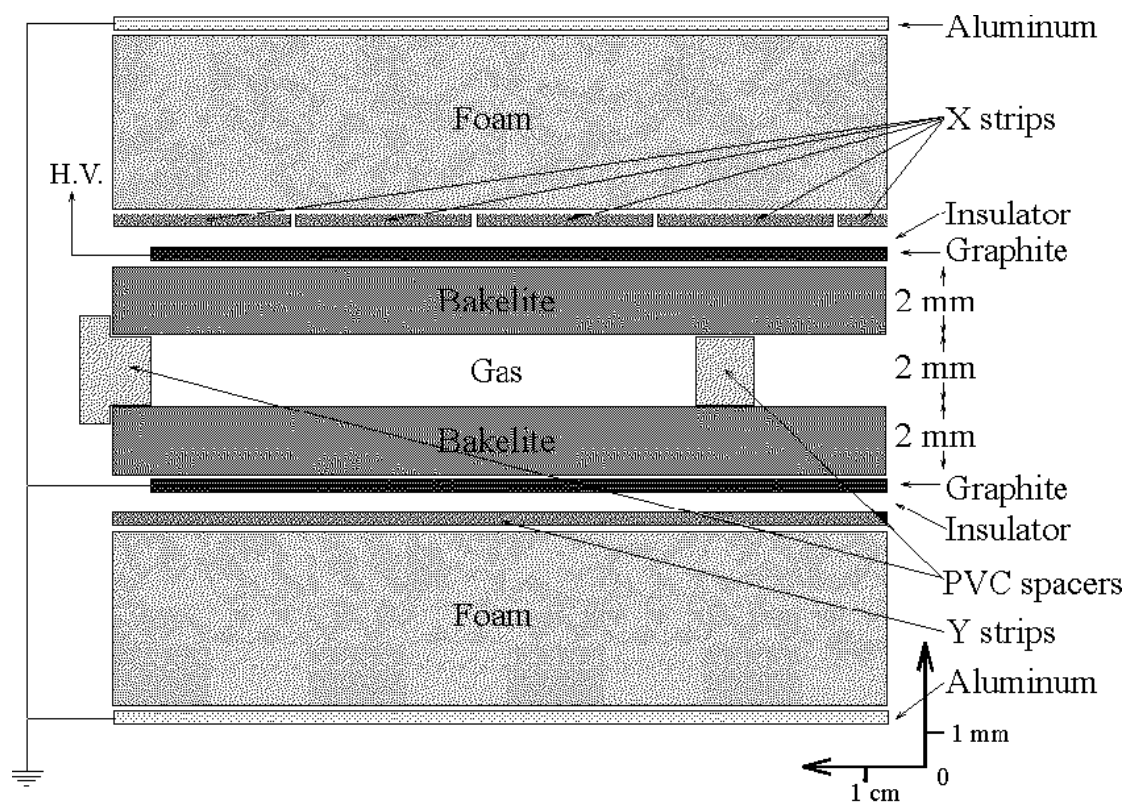

Figure 2.15: The BABAR Instrumented Flux Return: Design of the Resistive Plate Counters (RPCs) used by the IFR

The barrel modules consists of 32 horizontal strips per module. Strips with planes facing the ground side of the RPCs and which run along each module are used to measure the $\mathrm{z}$ coordinate. Each strip is $32.5 \mathrm{~mm}$ wide and a gap of $2 \mathrm{~mm}$ exists between the strips. The total pitch in this assembly is $38.5 \mathrm{~mm}$. The $\phi$ angle is measured by the strips that are glued across three modules on the High Voltage (HV) electrode. There are 96 such strips with a radially increasing pitch ranging from $19.7 \mathrm{~mm}$ to $33.5 \mathrm{~mm}$.

In the endcap, the $\mathrm{x}$ and $\mathrm{y}$ coordinates are measured by 64 horizontal and vertical strips. The vertical strips are glued across two modules and have a pitch of $38 \mathrm{~mm}$. The horizontal ones are $26.4 \mathrm{~mm}$ in width and are glued onto the HV side of the modules. These strips have a pitch of $38 \mathrm{~mm}$ and a gap of about $2 \mathrm{~mm}$ is present between them.

One side of the strips contain electrodes which are made of aluminium and are glued onto a plastic support that is coupled via a $4 \mathrm{~mm}$ thick insulator onto bakelite sheets. The other side contains a $40 \mu \mathrm{m}$ aluminium sheet which is connected to ground. This is insulated from the iron by a mylar sheet. One end of these strips is connected to the ground plane via a $2000 \Omega$ resistor. The other end is connected 
to the 16 channel Front End read out Cards (FEC). In the barrel, the FECs for layers 1 to 16 are positioned directly on the chambers. The odd layers are read out by six cards positioned at one end and the even layers are read out by six cards positioned at the other end. The setup is such that adjacent strips are read out by different cards. The FECs used to readout layers 17 to 19 and the cylindrical RPCs are mounted on external mini-crates that are positioned on top of the barrel. In the endcap, the FECs used to read out the horizontal strips are positioned on the chamber while the others are mounted on external minicrates located on the doors.

\subsubsection{The trigger system}

The BABAR trigger [30] consists of a hardware level (Level 1 - L1) and a software level (Level 3 - L3). The L1 trigger is designed to have a very high efficiency and consists of the drift chamber trigger (DCT), the calorimeter energy trigger (EMT), the IFR muon trigger and the global trigger (GLT). The DCT accepts tracks based on short or long track lengths and high $p_{\mathrm{t}}$ primitives. The EMT requires at least two clusters in the EMC with an energy deposit greater than $120 \mathrm{MeV}$. The above trigger runs at around $800 \mathrm{~Hz}$ and yields about $100 \%$ efficiency for B physics. The information from the various trigger elements are processed by the GLT. It is the GLT that sends information to the Data Acquisition System (DAQ) on when data are to be read out.

The L3 trigger uses data from the DCH/DCT and the EMC to construct track and cluster objects. The L3 decision process, with the exception of Bhabha events, is determined by the overall topology of the tracks/clusters and not by individual physics processes. The full physics trigger is a logical OR of two independent filters, the track filter and the cluster filter. The cluster filter, as presently implemented, accepts events with high energy deposits in the EMC and a high invariant mass or ones with a high multiplicity. The track filter, on the other hand, requires either a track originating from the IP with a $p_{\mathrm{t}}>800 \mathrm{MeV} / \mathrm{c}$ or two tracks with $p_{\mathrm{t}}>$ $250 \mathrm{MeV} / \mathrm{c}$ with rather loose vertex requirements. 


\section{The trigger performance}

The level 3 output event display showing a multi-hadron event is given in figure 2.16. A definition of the L1 trigger primitives can be found at [38] and the L3 trigger is described in [39]

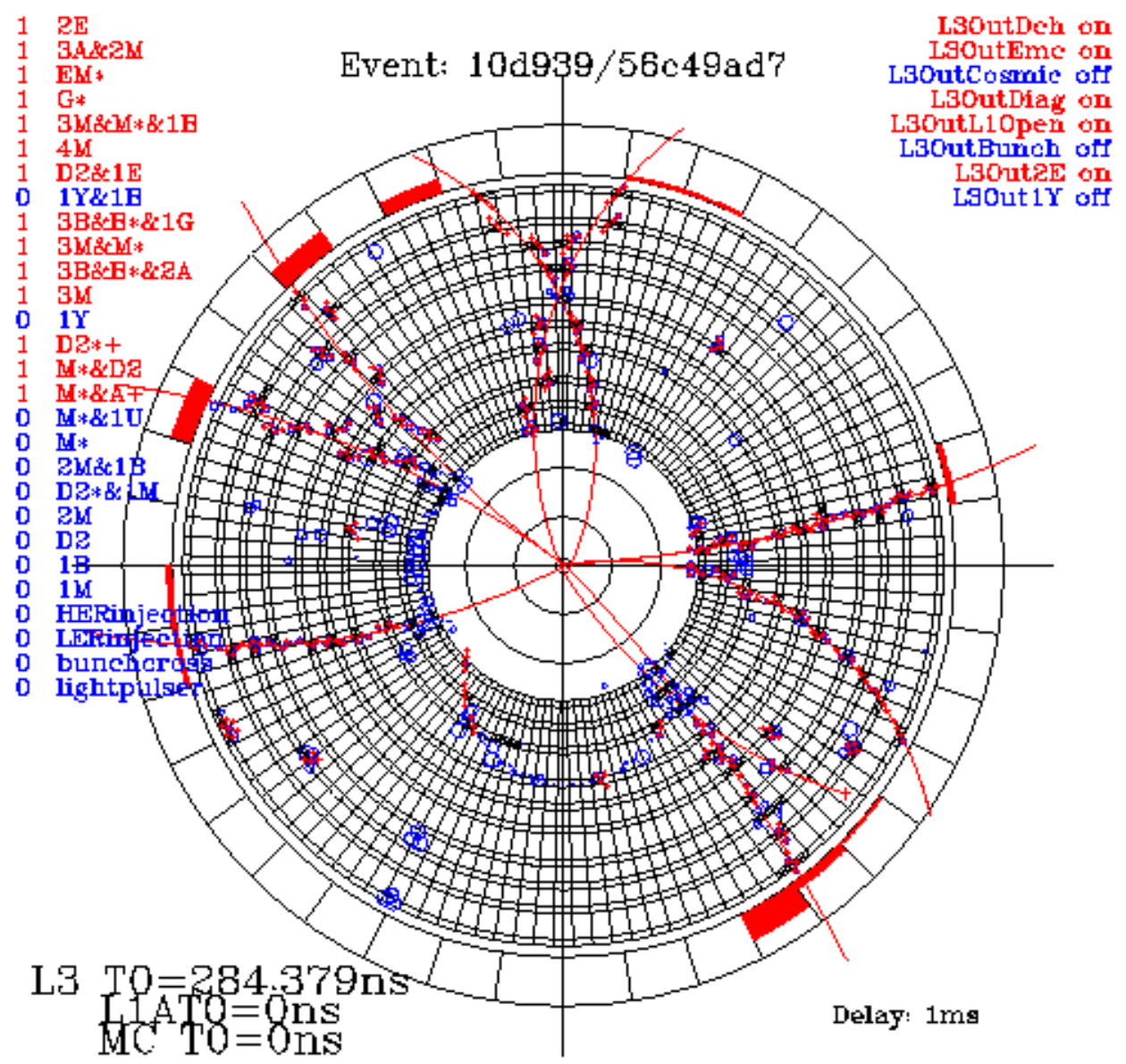

Figure 2.16: The BABAR L3 Trigger Display: L3 output showing a muli-hadron event. The L1 and L3 trigger lines are given on the left and right respectively.

\subsection{Chapter summary}

An overview of the PEP-II B factory and the BABAR detector has been given. The $B A B A R$ detector was commissioned in 1999 and has been successfully recording data. The performance of the BABAR sub-detector components have been discussed. 


\section{Chapter 3}

\section{The calorimeter readout system and simulation}

\subsection{Introduction}

The Data Acquisition System (DAQ) is a key component of any high energy physics experiment. An highly efficient and effective DAQ system is crucial to utilize the full physics potential of any experiment. A successful DAQ system is an intimate partnership between the hardware responsible for recording the data and the software that drives the functionality of the hardware. An accurate simulation of the detector is required to fully understand the physics potential and the detector performance of any experiment. A successful detector response simulation is one that is an accurate representation of the detector and the DAQ system.

The aim of this chapter is twofold. Firstly, to give the reader an understanding of the BABAR DAQ system [40] and secondly to present a discussion of the BABAR simulation software that tries to emulate the DAQ system. The overview of the BABAR DAQ system will be presented first. This will be followed by a discussion of the functionality specific to the EMC. This section is to be considered an extension to the EMC discussion presented in chapter 2. The latter part of the chapter is devoted to the BABAR detector response simulation software. This will contain an overview of the general BABAR simulation framework and a discussion of the EMC front-end electronics simulation. 


\subsection{BABAR DAQ system}

\subsubsection{Introduction}

The very high luminosity and beam crossing rates delivered by PEP-II put stringent requirements on the DAQ system implemented by BABAR. Not only does the DAQ system have to deal with a large number of readout channels but has to do so at a design Level 1 (L1) output rate of $2 \mathrm{kHz}$. The minimization of the dead time of the DAQ is thus a crucial design aspect of the system. An attractive feature of the BABAR DAQ system is its multi-functionality. It has been designed with the ability to run in multiple platforms. A platform, also known as a dataflow platform, is an assembly of hardware components that utilize the DAQ software (e.g. EMC test stand, SVT test stand and the final detector assembly). In this section, the data taking detector assembly platform will be referred to as the IR-2 Platform. The DAQ system has the capability to simultaneously run multiple subdetector components in multiple configurations. For example, it is therefore possible to calibrate one detector element (e.g. EMC forward end cap) whilst taking data with other components (e.g. a run taking cosmic ray data using the IFR and the trigger system).

\subsubsection{DAQ architecture}

Before a description of the DAQ system is presented, an overview of the relevant terminology will be given. The software used for data taking will be referred to as dataflow. The hardware component of the DAQ system, as previously stated, is known as the dataflow platform. Hardware components used for driving the DAQ process and for the collection of data are referred to as dataflow modules. The dataflow modules are housed in standard VME crates [41]. Two types of crates, the DAQ master crate and the DAQ slave crates, are used at BABAR. A given platform has at most one master crate which is used to control the slave crates within that platform. The slave crates, on the other hand, host the modules used to control and gather data from the front end electronics. The primary aim of the slave crates is thus to facilitate this data taking process which is controlled by the master crate. It should be noted that it is also possible to have a stand alone slave crate within a 
given platform. This set up is used in the sub-system test stands. The IR-2 Platform is set up using the master-slave crate assembly and it will be described next.

\section{DAQ master crate}

The control of the slave crates by the master crate is accomplished by the Fast Control Timing System (FCTS) modules [42] that are housed within that crate. These modules are used to create and distribute timing and control signals to the rest of the DAQ platform. The full assembly of the DAQ modules in the IR-2 platform is shown in figure 3.1. The following FCTS modules are housed in the master crate.

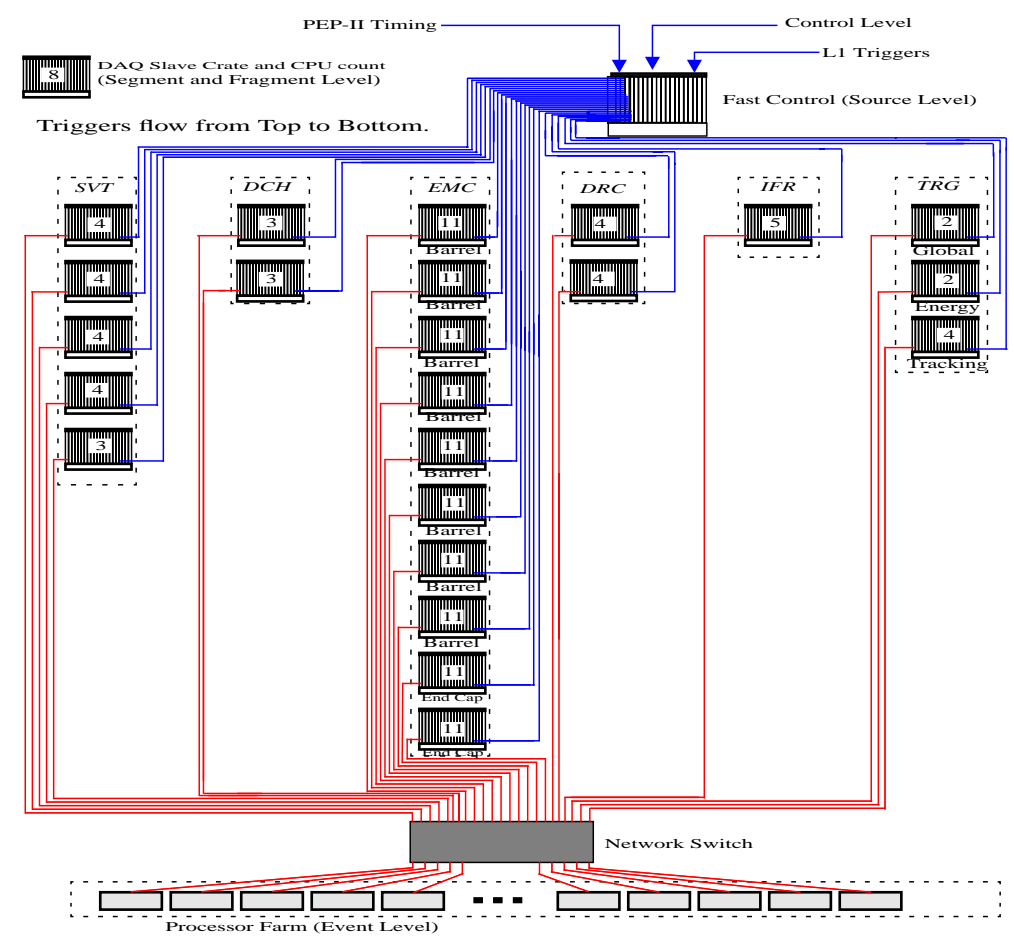

Figure 3.1: The BABAR DAQ System : The full assembly of all DAQ modules within the IR-2 platform. The EMC, with $110 \mathrm{DAQ}$ modules is by far the largest subsystem.

- The Fast Control Timing Module (FCTM) - This module is responsible for deriving the time (sysclk and subFiducial) used by BABAR. The sysclk is used to synchronise the data taking by the various slave crates and is derived from the $476 \mathrm{MHz}$ RF clock and fiducial marker from PEP-II [43]. The FCTM 
is also responsible for monitoring the relative alignments of the sysclk and the subFiducial during data taking. It also has the added functionality to synthesize a sysclk should the DAQ system need to be exercised in the absence of the PEP-II system (e.g. a detector calibration run).

- The Fast Control Gate Module (FCGM) - The interface to the BABAR trigger system is provided by the FCGM. The trigger signals received by the FCGM are then distributed to the rest of the DAQ system. It also has the ability to mask or scale various trigger lines during data taking. High frequency events such as Bhabha events can thus be suppressed such that a reduction in the load on the DAQ system and the subsequent reconstruction system can be achieved.

- The Fast Control Partition Master (FCPM) - The assignment and management of DAQ partitions is accomplished by the FCPM. A given FCPM can manage a partition of one or more slave crates. In doing this, the FCPM uses command packets to transmit instructions. A command packet is a 104 bit word that is transmitted at $1 \mathrm{bit} / \mathrm{sysclk}$. The transmission of an entire command packet thus takes $1.73 \mu \mathrm{s}$. The L1Accept, which defines an event accepted by the L1 trigger system, is an important example of such a command packet issued by the FCPM. The FCPM is also used to test the data taking readiness of the slave crate modules by means of full/available lines. A slave crate will issue a full line when it is not in a state for data taking. An available line, on the other hand, is issued to signify data taking readiness. The FCPM can reside in either the master crate or, in the case of a stand-alone platform, in a slave crate.

- The Fast Control Partition Router (FCPR) - The command packets issued by the FCPM and the sysclk issued by the FCTS are transmitted to the slave crates by the FCPR. The router is also used to feed in the full/available lines from the slave crates into the FCPM. In the case of a stand alone platform where the FCPM resides in a slave crate, a router is not required. 


\section{DAQ slave crate}

The DAQ slave crate in the IR-2 platform houses the following dataflow modules.

- The Fast Control Distribution Module (FCDM) - All communications between the master crate and a slave crate are sent through the FCDM. This communication is a two way process. The FCDM receives the sysclk and FCTS command packets from the FCPR which are then distributed to the Read Out Modules (ROM) housed within that crate. The full/available lines from the ROMs are gathered by the FCDM and are sent on to the FCPR.

- The Read Out Module (ROM) - The ROMs are used to control and gather information (both data and diagnostic information) from the Front End Elements (FEE) on the BABAR detector.

\section{The Read Out Module (ROM)}

A schematic diagram of the BABAR ROM can be seen in figure 3.2. The ROM consists of a Controller Card (CC), a Single Board Computer (SBC), a PCI Mezzanine Card (PMC) and a Personality Card (PC). The CC is used to provide the interface to the FCTS and it receives the sysclk command packet from the FCDM in that crate. This is then distributed to the FEE under its control. The CC also uses the L1Accept signal to initiate the data transfer from the FEE or to transfer (using DMA- Direct Memory Access) the data to the SBC. In the absence of FCTS, the CC can be used to synthesize a sysclk and thus enable the ROMs to be run in a test stand environment. BABAR uses an Intel i960 based PMC card. The main functionality of this is to manage the transfer of event data from the PC to the SBC and the transfer of FCTS signals from the CC to the SBC. The SBC core is a Motorola Power PC (PPC) chip based processor card running the VxWorks real-time operating system. The PC comes in two flavours; the Untriggered Personality Card (UPC) which is used exclusively by the EMC and the Triggered Personality Card (TPC) used by all the other subsystems. It should be noted that all subsystems including the EMC use a TPC ROM to control the data gathering ROMs within a slave crate. A description of the UPC ROM can be found in section 3.3.2. 


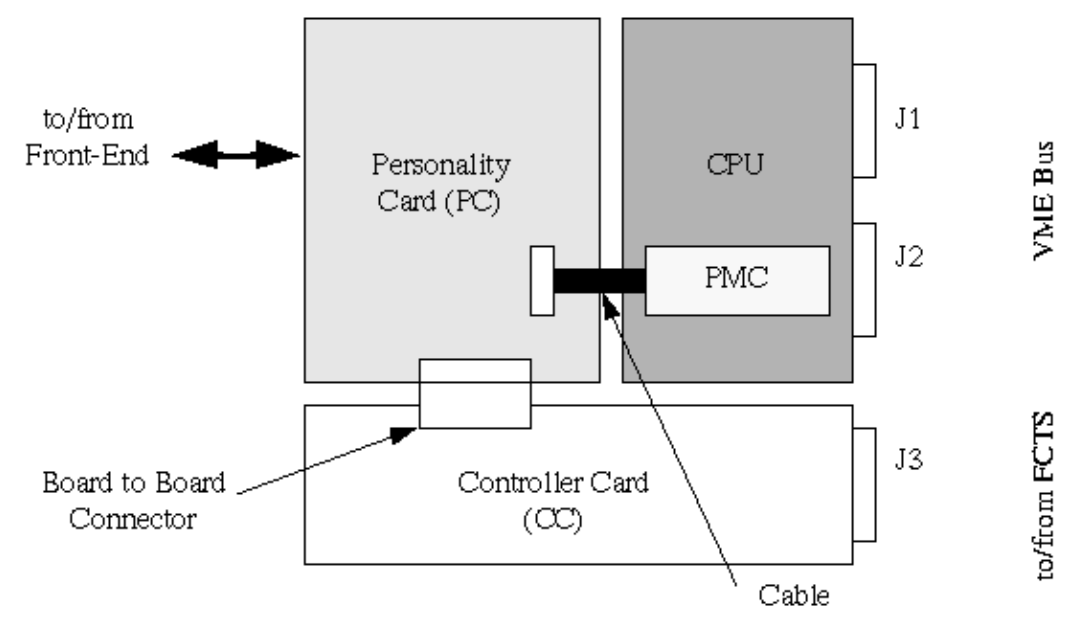

Figure 3.2: The BABAR DAQ ROM : A schematic of the BABAR ROM with a generic PC showing the connections between the individual components. The communications with the FEE are handled by the PC. The FCTS signals are received by the CC via the J3 backplane.

\subsection{The EMC front end electronics and DAQ sys- tem}

\subsubsection{EMC electronics}

The EMC front end electronics transmit a continuous stream of data to the ROMs. As a result, as previously stated, the EMC uses ROMs with the untriggered version of the personality cards. This data transmission rate is driven by the $3.74 \mathrm{MHz}$ speed of the Analogue to Digital Converters (ADCs) used by the electronics. A full description of the EMC front end electronics can be found at [44]. The produced scintillation light is picked up by two photodiodes that are mounted on the rear faces of the $\operatorname{CsI}(\mathrm{Tl})$ crystals and are then sent to a pre-amplification stage. The output of the pre-amplification is sent through a $C R-R C-R C$ shaping integrated circuit. The output of this is then sent via $\mathrm{x} 1$ and $\mathrm{x} 32$ gain amplifiers to a 10 bit Custom Analogue Range Encoding (CARE) chip. The CARE is responsible for encoding the data into $\mathrm{x} 1, \mathrm{x} 4, \mathrm{x} 32$ and $\mathrm{x} 256$ gain ranges. The output of the CARE is a 12 bit word (10 bit value and 2 bits signifying the amplification range). Each CARE chip analyses the data from 24 channels. The CARE output is converted by a Finisar transmitter into an optical signal which is then sent to the ROMs through a fibre optic link. The CARE/transmitter assembly is mounted on an 
Input Output Board (IOB). The IOBs in the EMC barrel, Barrel IOBs (BIOBs), consists of three CARE/transmitter units whilst the endcap IOBs (EIOBs) contain two CARE/transmitter combinations.

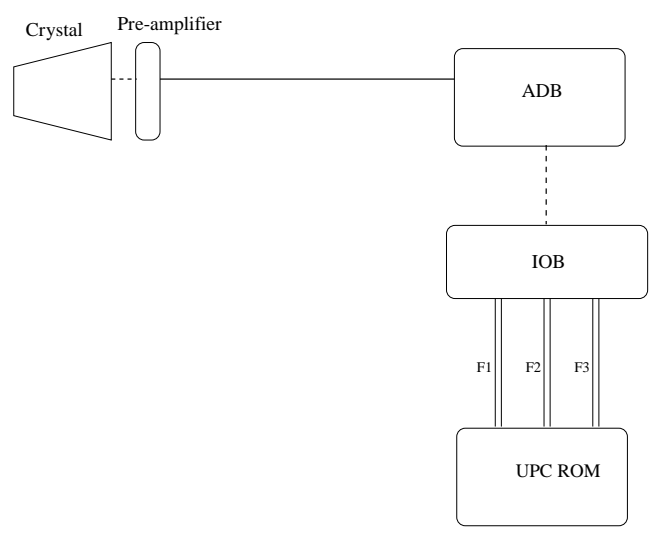

Figure 3.3: The BABAR EMC READOUT CHAIN: A schematic showing the connections from an EMC crystal to the ROM. Each IOB reads data from two Analogue to Digital Boards (ADBs) . The data are then sent via a fibre optic link (FLINK) to the ROMs in a DAQ crate. A barrel $I O B-R O M$ combination that utilizes three FLINKs is shown in this diagram.

The data are transmitted every $269 \mathrm{~ns}$ in the form of a packet which is made of sixteen 20 bit words, figure 3.4. The length of an entire packet is then 320 bits. Of this, 308 bits are reserved for the 12 bit CARE outputs of the 24 channels. The rest consists of the fibre link number, the IOB serial number and other information used for diagnostic purposes. The IOBs reside on the BABAR detector.

\subsubsection{The UPC ROM}

The data which are sent from the the IOBs are received, deformatted and analysed by the UPC ROMs. In this section, the basic functionality and operation of the UPC ROM will be given. A comprehensive discussion of the UPC ROM can be found at [47].

The data packets from the IOBs are received by FLINK receivers. A UPC board can have up to three FLINK receivers (FLINK A, FLINK B, FLINK C). A UPC receiving data from a BIOB utilizes all three FLINKs whereas an endcap one requires just two FLINKS (FLINK A and FLINK B). A one-to-one mapping thus exists between the IOBs and the UPCs. Upon the receipt of the data packets, 


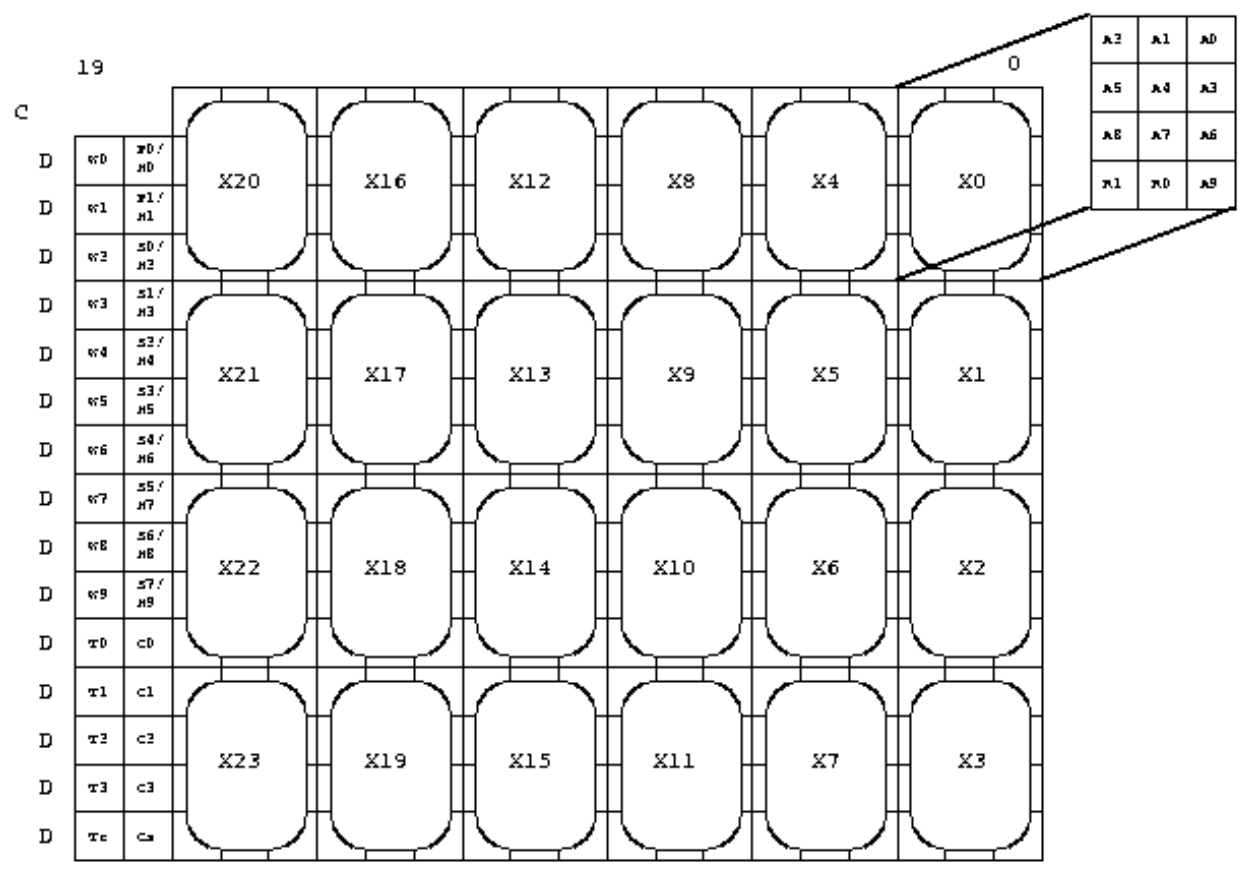

Figure 3.4: The Format of the data packets sent by the EMC FEE to the UPC ROM. Each packet contains data from 24 crystals (X0-X23) and diagnostic information. Each crystal energy is given by a 10 bit word (A0-A9) and two further bits $(R 0, R 1)$ that are used to specify the gain range used by the CARE.

they are first deformatted by means of a deformatter chip. As the EMC uses four amplification ranges, before any of the raw ADC data can be used in calculations, they have to be converted to a common scale. The deformatted 12 bit raw ADC counts are therefore sent to a Look Up Table (LUT), where they are converted to an absolute energy scale. The output of the LUT is a 16 bit unsigned integer such that the least significant bit corresponds to $250 \mathrm{keV}$. As the pre-amplifiers used in the IOBs are AC coupled, it is possible for the ADC pulse to have a negative undershoot. Thus, to take into account this undershoot in the unsigned integer, an offset is added during the LUT stage. Note that it is possible to define the LUT output as a signed 16 bit integer but this reduces the dynamic range to 15 bits. To effectively preserve the full 16 bit dynamic range and allow for the negative undershoot, the LUT offset is therefore used. The output of the LUT are sent to the calorimeter trigger (EMT) system and to an intermediate store (IS). The LUT also generates two flags, ADD and FEX for each crystal. The ADD bit is used by the output sent to the EMT, whilst the FEX bit is used to define which crystals are used in the feature extraction 


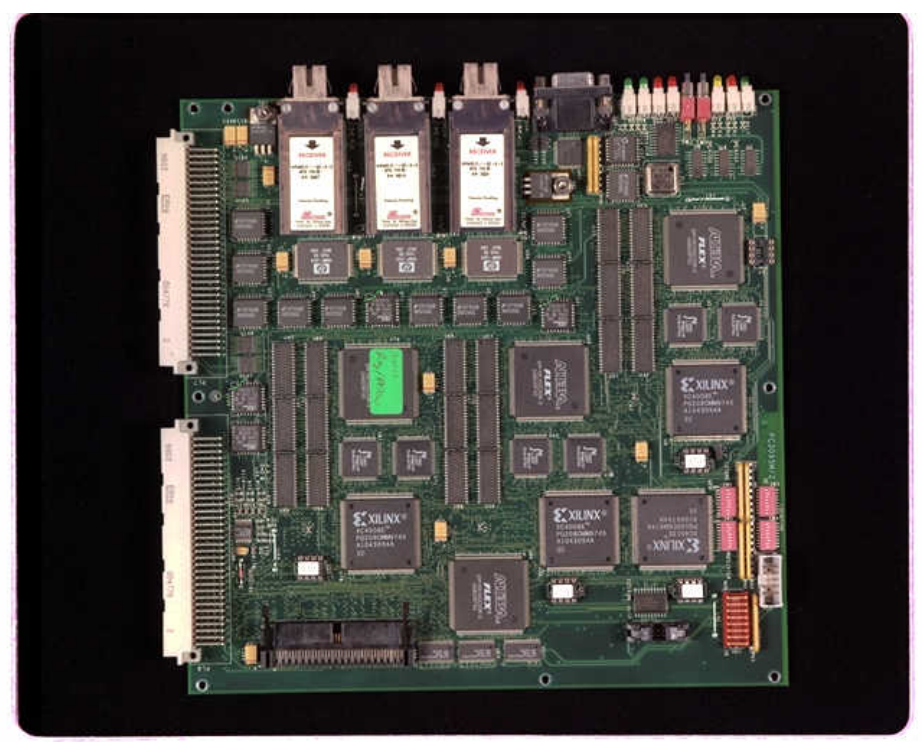

Figure 3.5: The BABAR EMC UPC : A picture of the UPC ROM.

process in the SBC.

\section{UPC-Trigger interface}

The EMT uses the crystal energies obtained by the EMC in deriving trigger decisions. The EMT, in fact, uses the total energy of all the good crystals served by a FLINK rather than individual crystals when making trigger decisions. This sum, which has to be provided by the UPC, corresponds to a single EMT tower. This summation is done by a Trigger Summer (TS) which is an EPROM (Erasable Programmable Read Only Memory) chip. When doing this summation, it is imperative that dead or noisy crystals are not included. This is accomplished by the ADD bit mentioned above. Only crystals which have the ADD bit set are used in the tower sum generation. The LUT offset which was added during the LUT conversion is, of course, deducted when generating the tower sum. Before the data are sent to the trigger, they are synchronised to the sysclk. The following data are then sent to the trigger: the tower sums for each TS, the common sysclk used to synchronise the tower sums and a FRAME bit which is used to indicate the start of the word. Each UPC to EMT data transfer occurs at a rate of $59.5 \mathrm{Mbits} / \mathrm{s}$. 


\section{The UPC intermediate store}

The output of the LUT conversions are also stored in an intermediate store (IS) within the UPC before being sent to the SBC. The data from the 24 crystals corresponding to a FLINK are stored as eight 64 bit words. Once again, a one-to-one mapping exists between the FLINK and IS. A schematic diagram of the IS can be seen in figure 3.6. Each 64 bit word contains information about three crystals and consists of the following: the 16 bit output of the LUT, the ADD and FEX bits for each crystal and the trigger summer output sent to the trigger. Two other bits, LINKREADY/ERROR and the END bit are also set. The LINKREADY/ERROR is used to signify the data taking readiness of the link. It is set if the link was not ready or died during data taking. The END bit, which is bit 63 , is used for diagnostic purposes and is checked after the DMA of the data from the IS to the SBC.

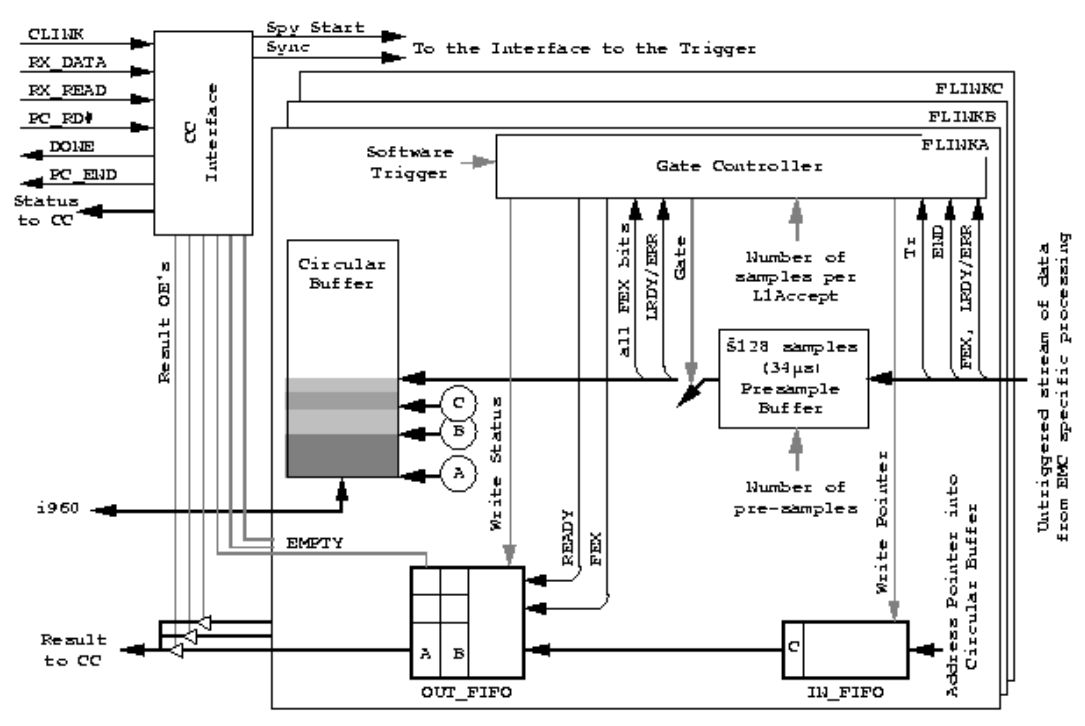

Figure 3.6: The BABAR EMC UPC : A schematic of the UPC Intermediate Store. On receipt of an L1Accept, the pre-sampler to circular buffer gate is closed and data are transferred into the circular buffer. This process is controlled by the gate controller.

\section{The SBC and feature extraction}

Following the DMA of the data to the SBC after a L1Accept, the data are feature extracted. The output of this process is sent to the Online Event Processing 
(OEP) system via an Ethernet connection where further processing and data quality monitoring takes place. The output of this stage serves as input to the BABAR reconstruction system.

\subsection{The EMC front end electronics simulation}

The EMC front end electronics are simulated in the $\mathrm{C}++$ programming language within the BABAR simulation framework. The simulation source code resides in the EmcSim package of the BABAR software. The simulation tries to emulate the full extent of the electronics and the DAQ system of the detector. An overview of the EMC electronics simulation will be given in this section. This will be followed by a description of the implementation of machine background noise within the BABAR simulation environment. The section will end with a description of a polynomial fit which can be used in conjunction with the filter to improve the overall resolution of the readout system. It is assumed that the reader is familiar with the techniques of Object Oriented (OO) design and, in particular, comfortable with the concept of an object. A detailed discussion on OO programming techniques can be found elsewhere [48].

\subsubsection{The BABAR simulation}

The $B A B A R$ simulation software is designed to provide an accurate description of the $B A B A R$ detector and the PEP-II interaction region. The energies of the high and low energy beams are smeared by Gaussian distributions with widths of $5.5 \mathrm{MeV}$ and $3.1 \mathrm{MeV}$ respectively. The beam spot positions in $\mathrm{x}$ and $\mathrm{y}$ are also smeared by Gaussian distributions whilst the beam spot position in $\mathrm{z}$ is modelled as a flat distribution which is $1 \mathrm{~cm}$ long. BABAR uses two event generators, EvtGen [49] which is the main generator for $B^{0} \bar{B}^{0}$ and $B^{+} B^{-}$events and Jetset7 .4 [50]. EvtGen has the ability to generate a large number of physics processes in great detail and also has the ability to handle asymmetries in CP violating decays. Mixing between the $\mathrm{B}$ mesons is handled by generating $\Upsilon(4 \mathrm{~S})$ decays into the correct mixture of $B^{0} \bar{B}^{0}$, $B^{0} B^{0}$ and $\bar{B}^{0} \bar{B}^{0}$ states with the correct distributions of the proper decay times. The core of the BABAR simulation software is the Geant321 [52] based BBsim package. 
The BBsim package is interfaced to the event generator via the Beget package [51] which simulates the smearing of the interaction point and the beam spot as described above. Given a geometrical description of the detector and the 4 -vectors of the $\Upsilon(4 \mathrm{~S})$ decay products, the BBsim program outputs a list of subsystem specific energy or charge deposits (GHits). The GHits are then used by the subsystem specific front end electronics simulation software.

\subsubsection{EMC simulation}

The EMC front end electronics simulation uses as input the EMC specific BBsim hit, the EmcGHit. An EmcGHit object contains information about the energy deposited by a particle within a given EMC crystal. This includes, amongst other things, the energy deposited, the time of the deposit and the $\theta$ and $\phi$ indices of the crystal. An EmcGHit is to be thought of as the simulation equivalent of the scintillation light emitted by particle interactions with the CsI crystals.

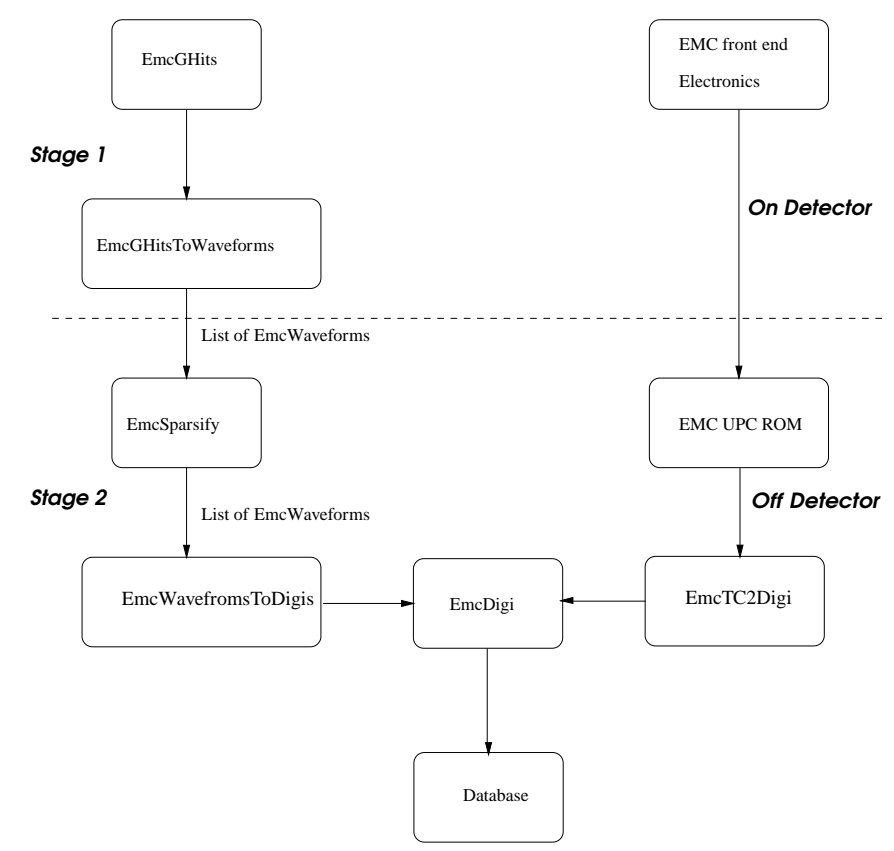

Figure 3.7: Functional diagram of the EmcSim package showing the modules used in the conversion of EmcGHit objects into digi objects. The EmcGHits are first converted into intermediary waveforms which are sparsified before being feature extracted. The equivalent process being carried out in the real detector is shown on the right.

The simulation consist of two main stages, figure 3.7. In the first stage, the EmcGHits are smeared into waveforms (EmcWaveform objects) according to the 
$C R-R C-R C$ shaping times of the front end electronics. This is performed by the EmcGHitsToWaveform module in the EmcSim package. A given waveform has a length of 64 time bins, with one time bin $\approx 270$ ns. This corresponds to the ADC rate of $3.74 \mathrm{MHz}$. The time in this case is measured relative to the beam crossing time, which is given by the fast control timing simulation. In the creation of the EmcWaveform objects, noise due to electronics and digitisation of the analogue pulse are added. As electronic and digitisation noise affects the readout of all the crystals, an EmcWaveform is thus created for each crystal. The impact on a waveform by an EmcGHit is then calculated and added onto the waveform. If a given crystal contains multiple EmcGHits (e.g. due to machine background noise), the impact on that crystal's waveform will be calculated for each EmcGHit and then added to that waveform. The next stage involves the addition of machine background noise onto the signal waveforms. Presently, there exists two distinct ways of handling this within the simulation framework. These are described in section 3.4.3.

The final section in this pipeline is the sparsification and feature extraction stage. This stage, referred to as feature extraction, is a description of the processes that are carried out on the SBC of the UPC ROM, figure 3.7. Before the waveforms are digitally filtered, they are sent through a sparsification algorithm. The motivation for this is to remove the isolated hits due to noise and thus obtain a reduction in the load on the filtering algorithms. The sparsification algorithms reside in the EmcSparsify module and work as follows. If a given crystal contains a waveform that peaks at an energy above the sparsification threshold, presently $10 \mathrm{MeV}$, that waveform and the ones belonging to the nearest neighbours of that crystal are kept. The waveforms that pass this sparsification stage are filtered using a matched digital filter and subsequently converted into digis by the EmcWaveformsToDigis module. A full description of the digital filter algorithms can be found elsewhere [54]. The matched filter uses a set of weights which are a function of the ideal (no noise) signal and the autocorrelation function of the noise spectrum. Noise in this context includes both electronic noise and background photon hits. The waveforms that are sent through the filter algorithm are then converted into digis. The simplest form of such conversion involves a simple peak finding algorithm. The peak finding algorithms consider just six bins $(-1$ to +5$)$ around the time bin corresponding to the Level 1 
time. The motivation for searching over 6 bins is two fold. Firstly, the L1 trigger time uncertainty is of the order of $\pm 500 \mathrm{~ns}$. This corresponds to an uncertainty of \pm 2 EMC time bins. Secondly, searching over the entire waveform length of 64 time bins is too CPU intensive. Thus, for code optimisation purposes, the algorithm was set up to search for the peak over 6 time bins around the L1 time. Recalling that the time in the EMC simulation is measured relative to the beam crossing time, the reason for the choice of -1 to +5 bins is due to a 500 ns offset in the L1 time relative to the beam crossing time. This is shown in figure 3.8. The energy and time of the original hit(s) are then taken to be the peak of the waveform and the time bin corresponding to the peak respectively. A more complicated form of waveform $\rightarrow$ digi conversion involves a second order polynomial fit which is described in section 3.4.4. These fit algorithms also reside in the EmcWaveformsToDigis module.

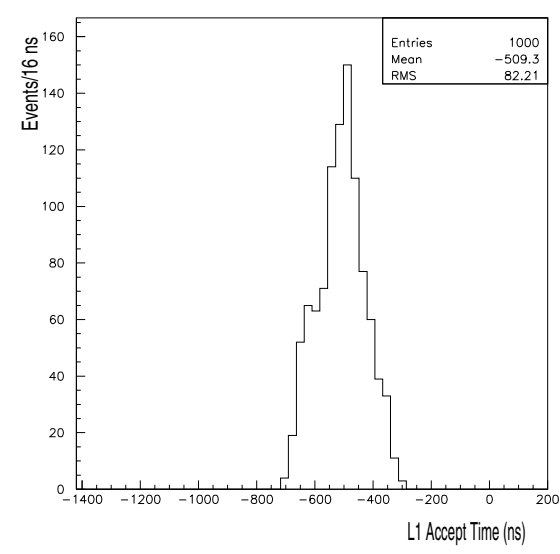

Figure 3.8: Level 1 time in the simulation. The distribution is centred around -500 ns.

The output of the EmcWaveformsToDigis module is an EmcDigi object. Each EmcDigi contains the energy, time and crystal index $(\theta, \phi)$ information. The digi objects, which are written by the simulation into the persistent store (database), serve as the input to the $B A B A R$ reconstruction algorithms.

\subsubsection{Machine background noise in the simulation}

An important feature of the simulation process is the inclusion of machine background noise. To do this, two methods were employed. The first, GHit mixing, uses 
xdr (eXternal Data Representation) files containing GHits derived from simulating background conditions in the machine. The other is a more complicated method that utilizes the BABAR OO database. This method, known as event mixing or digi mixing, has the ability to mix both simulated and real detector backgrounds. The real detector backgrounds are random events that were triggered by a $1 \mathrm{~Hz}$ trigger. The aim of the section is to highlight the two processes and to give a detailed description of their implementation within the EMC simulation framework. The GHit mixing process will be presented first.

\section{GHit mixing}

The backbone of this method are the mixing modules which smear the times of the simulated GHits into $12 \mu$ s frames. This is done by the trgFrame module and a description of this can be found elsewhere [55]. The input to the GHit mixing simulation are thus two xdr files of GHits. One containing the GHits from a simulated physics process and the other the frame file described above. Both types of EmcGHits are then smeared into waveforms by the EmcGHitsToWaveform module as described in section 3.4.2. A waveform is thus created for each crystal and the contribution from each EmcGHit is then calculated and added to that waveform. The mixing between the physics process and the backgrounds therefore happens at this waveform stage. The motivation for mixing at this stage is as follows. Firstly, EmcGHits are points in energy-time space and there is no trivial way to add them up. Secondly, mixing at the waveform stage, though very CPU intensive, in principle gives a better representation of the real detector. As seen in figure 3.9 the background mixed waveforms are sent to the EMT simulation and to the sparsification and feature extraction stages of the EMC described in section 3.4.2.

\section{Event mixing}

The concept of event mixing is to mix real backgrounds with the simulated physics signals. The main motivation for this is to get an accurate description of the backgrounds within the simulation. But, nevertheless, this method also has the functionality to mix simulated backgrounds. A schematic of this mixing process can be seen in figure 3.10. The starting point in this process are then a collection of EmcDigis 


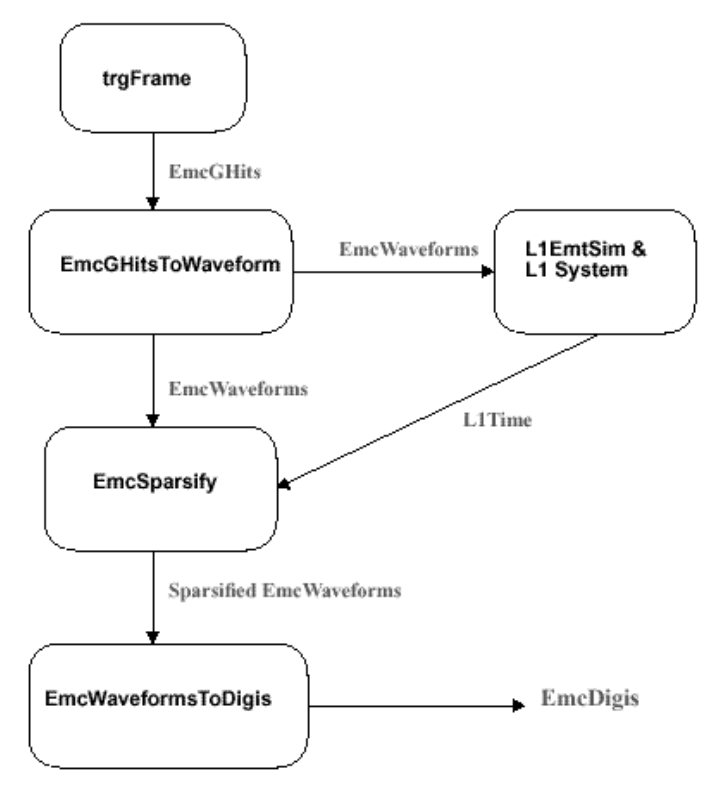

Figure 3.9: A diagram showing the GHit mixing procedure as implemented for the EMC. The MC backgrounds are mixed into the signal at the EmcWaveform stage by the EmcWaveformsToDigis module. The output of this is then sent the EMT simulation and to the EMC feature extraction stage.

in the database. It should be noted that these background digis can be from real data or simulation. When using simulated background digis, the entire simulation process has to be run to convert the background EmcGHits into digis. This can be done by running the GHit mixing algorithms over an empty physics file to generate a set of the background digis which can then be written to an appropriate database. As previously mentioned, the overlaying of background on the signal has to happen at the intermediary waveform stage. Therefore, the background digis have to be converted into waveforms. This reverse engineering process of digi to waveforms conversion is carried out by the EmcDigisToWaveform module. This module is based on the EmcGHitsToWaveform module and uses the same algorithms in doing the conversion. This is possible as EmcGHits and digis are points in the energy-time domain and the only two external inputs required in the EmcGHit/digi to waveform conversion are the energy and the time of the hit. However, this digi to waveform conversion is an approximation. It is very probable that the digi could have been a result of multiple EmcGHits. The background waveforms thus produced are mixed with the MC waveforms by the EmcMixWaveforms module. The procedure for 
doing this is similar to the one used by the EmcGHitsToWaveform module. Background waveforms for a given crystal are overlayed on the corresponding MC ones. The background mixed EmcWaveforms are then sent through the feature extraction process described before.

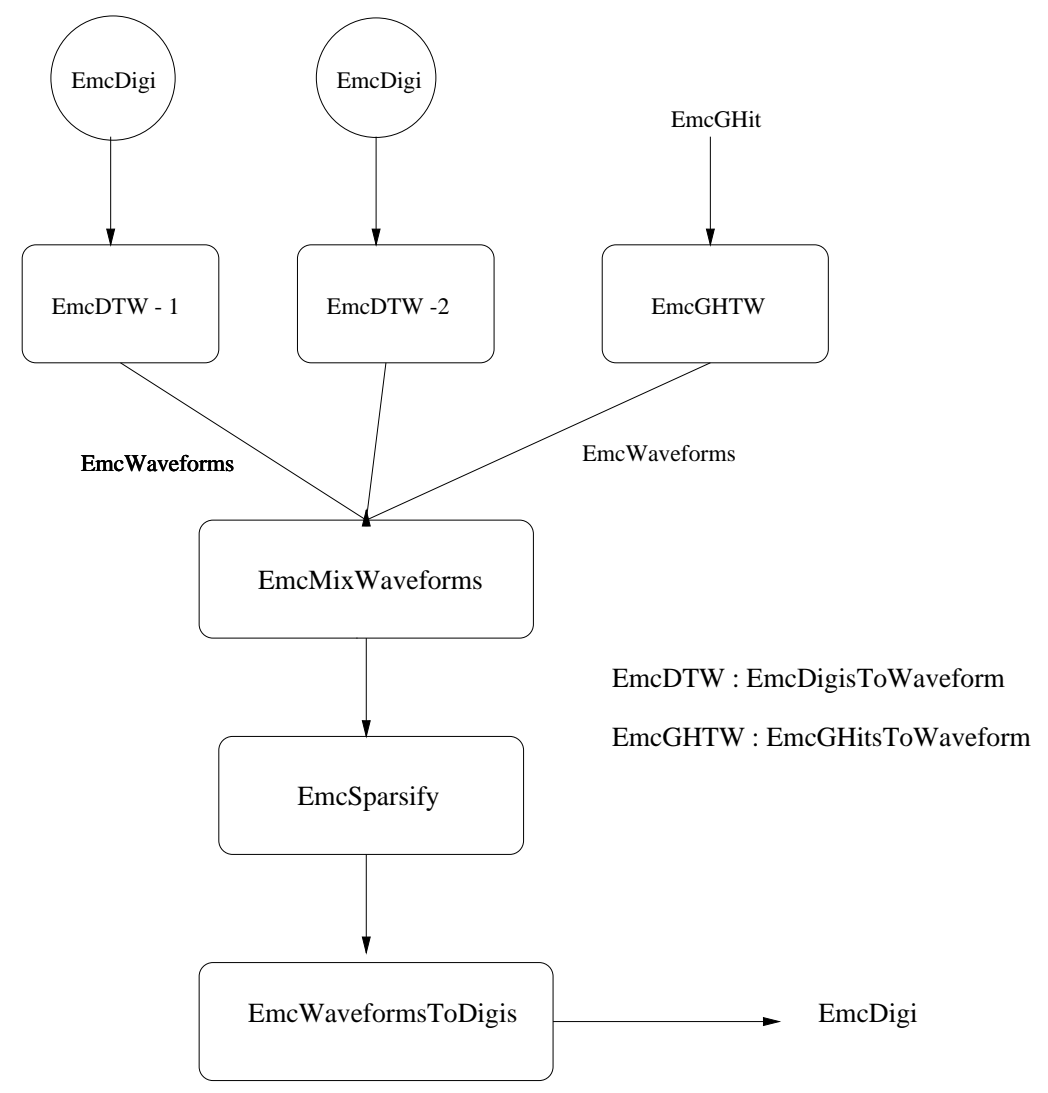

Figure 3.10: A diagram showing the event mixing process as implemented for the EMC. The starting point for the background events are a collection of EmcDigi objects in the database. These are then converted into waveforms and are then mixed with the MC signal.

Before a comparison with GHit mixing is given, a few words should be said about the features and limitations of the event mixing method as currently implemented. Firstly, as shown in figure 3.10, it has the functionality to mix multiple background collections. Secondly, as stated before, it also possible to mix in MC backgrounds. This provides a reliable method with which to validate the mixing procedure. A major limitation in the current implementation concerns the interface to the EMT simulation. Recall that in the GHit mixing process, figure 3.9, the background mixed waveforms were sent to the EMT simulation. In this process, however, the interface to the trigger simulation happens before the background mixing process (i.e. before 
the EmcMixWaveforms module). Thus, the trigger simulation receives the unmixed waveforms. The reason for this is related to the implementation of event mixing by the other subsystems. This limitation will be addressed in the next version of the event mixing software.

\section{Event mixing-GHit mixing comparison}

To validate the event mixing method, the event mixing algorithms were run using a MC generated background sample (frame file). First, the entire simulation process with the GHit mixer was run with a background MC file and a $\Upsilon(4 \mathrm{~S}) \rightarrow \nu \bar{\nu}$ file to generate the required background EmcDigis, which were written into the database. Next, the simulation process with the event mixing algorithms was run and then compared with the GHit mixer using the same background file. For both processes a generic $B^{0} \bar{B}^{0}$ decay file was used and the simulation was done for 100 events. Figure 3.11 shows the comparison plots for the total number of EmcDigis per events and the time of the EmcDigis. The distributions in figure 3.11 for GHit mixing is given by the solid histogram whilst the ones for event mixing are given by the dashed line. A good agreement can be thus seen between the two methods.

The digi energy distributions for the two methods can be seen in figure 3.12. Once again, the GHit and event mixing methods are given by the solid histogram and the dashed line respectively. A few words should be said about the structure of the energy distributions. The peak at -2 corresponds to the sparsification cut of $10 \mathrm{MeV}$ applied within the EmcSparsify module. The primary peak at around -3.4 corresponds to the electronics noise added to the MC waveforms. The electronics noise added was generated by assuming a Gaussian distribution with a width of $440 \mathrm{keV}$. As seen in figure 3.12 a discrepancy is present between the two cases around the electronics noise region. This is believed to be a result of the loss in resolution obtained in the EmcGHit $\rightarrow$ waveform $\rightarrow$ digi $\rightarrow$ waveform conversion that had to be done for the backgrounds in the event mixing case.

\subsubsection{Digital filter feature extraction}

\section{The performance of the digital filter}

The effects of the digital filter algorithms on the simulation were also investigated. For the filter to be a success, a smooth response over the entire energy range covered 

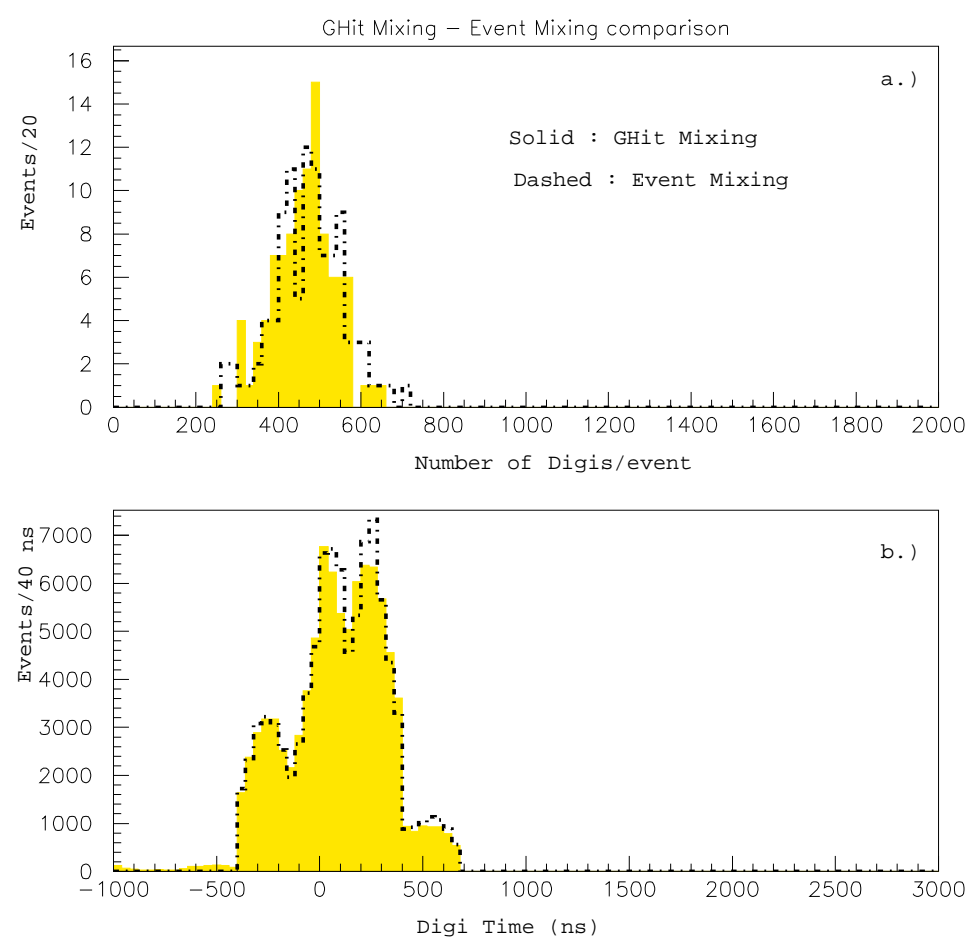

Figure 3.11: GHit mixing - Event mixing comparison plot showing the total number of digis per event (figure a) and the time distribution of the digis (figure b). The GHit mixing case is shown by the solid histogram and the event mixing by the dashed line.

by the EMC is required. The performance of the digital filter at different energies was thus considered. The EMC simulation with the filter algorithms was run for $30001 \mathrm{GeV}$ single photon events. With this it was possible to consider a wide range of energy deposits in the EMC. A cut in the sparsification algorithms of $10 \mathrm{MeV}$ was also used. Simulated backgrounds were mixed in via the GHit mixing process described above. The difference between the digi energies obtained and the input EmcGHit energies can be seen in figure 3.13.

The digi-EmcGHit energy distributions were found to be centred near zero with a small negative bias. It should be noted that this bias is due to the loss of information in the EmcGHit $\rightarrow$ waveform $\rightarrow$ digi process. The bias is not due to the filter as it was seen in the case of the feature extraction without the filter. The fall in the number of entries at higher energies, is of course, due to the decrease in the probability of getting an energy deposit with that particular energy in a single 


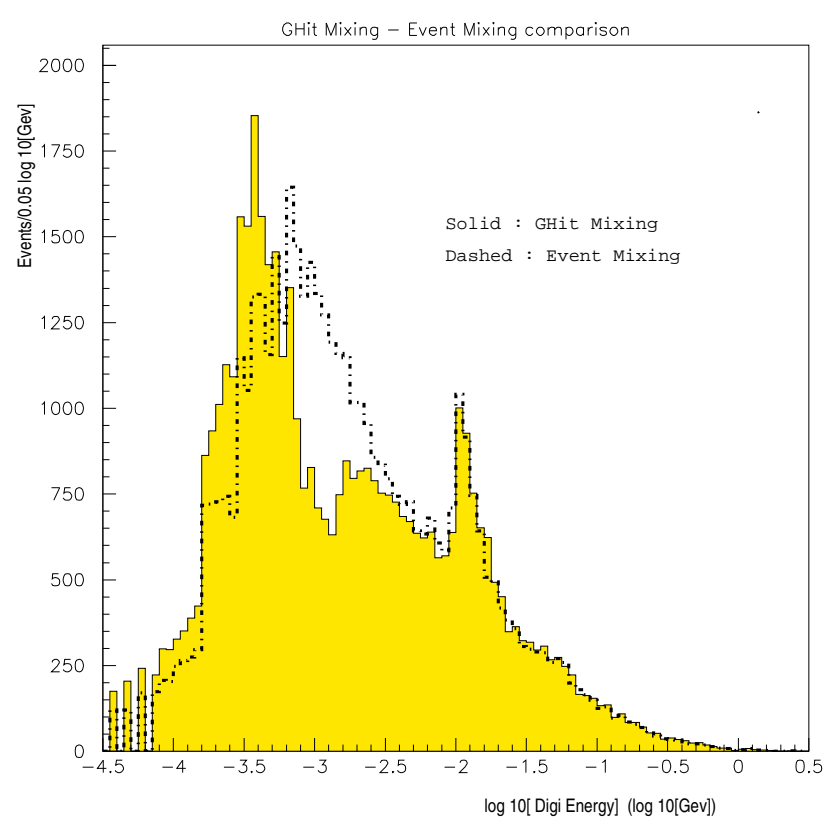

Figure 3.12: GHit mixing - Event mixing comparison plot showing the energy spectrum of the produced digis. The distribution for Ghit mixing is given by the solid histogram and that from event mixing by the dashed line.

crystal. The observed energy resolution $\left(\frac{\sigma_{E}}{E}\right)$ as a function of energy can be seen in figure 3.16.

\section{Second order polynomial fit}

The possibility of increasing the resolution of the filtered energy by using an appropriate fit was investigated [56]. The distribution of the maximum energy in each time bin for a given event was plotted for time bins of \pm 5 around the maximum for different energy ranges. The result of the fit can be seen in figure 3.14. The distributions were found to be Gaussian with a peak around time bin 48 .

For the case of the EMC online system an 11 point fit using a Gaussian is not feasible, as the filter algorithms are implemented in the ROM. Here, the CPU time taken to do the feature extraction is a crucial parameter. As a result, the improvement in resolution obtained by using a second order polynomial fit was investigated. The polynomial was solved using the maximum of the filtered waveforms and the two bins either side of it (Appendix A). As before, the simulation was run over 3000 

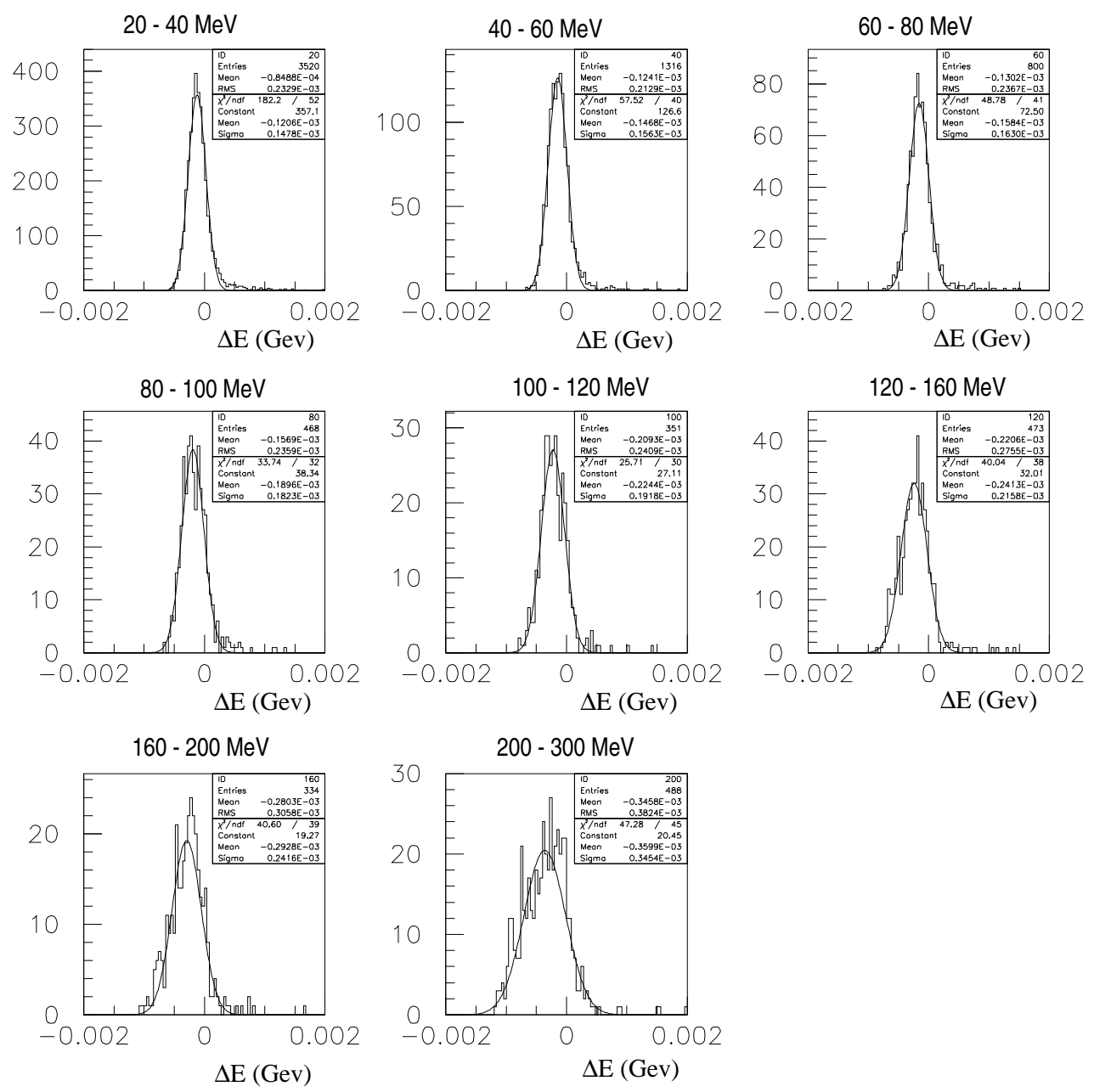

Figure 3.13: The difference between the EmcGHit energy and the digi energy for different Em$c$ GHit energy ranges. The $y$ axis in all the plots is in events $/ 0.04 \mathrm{GeV}$. This is before the polynomial fit is applied.

single $1 \mathrm{GeV}$ photon events. The difference between the digi and EmcGHit energies are shown in figure 3.15 .

Doing a comparison before (figure 3.13) and after (figure 3.15) the fit, a clear improvement can be seen. The comparison of the obtained energy resolutions as a function of energy is shown in figure 3.16. Here, the resolutions before and after are given by the boxes and triangles respectively. An improvement in the resolution of about $3 \%$ for $30 \mathrm{MeV}$ photons and of about $40 \%$ for $700 \mathrm{MeV}$ photons was observed. It should be noted that this improvement is on top of the improvement in resolution obtained by using the digital filter alone. 

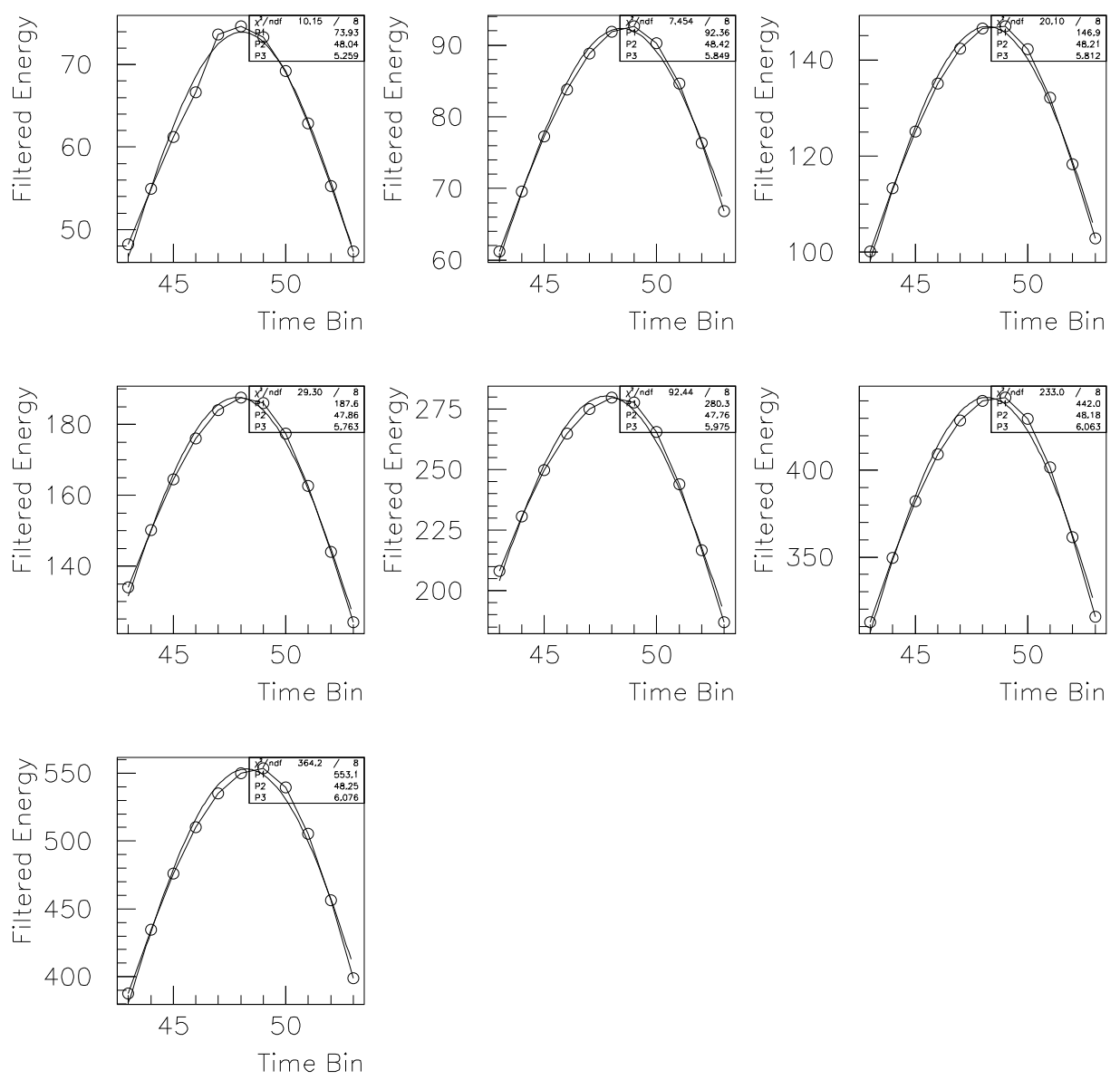

Figure 3.14: Digi energy in MeV vs. time bin plots with a Gaussian fit over 11 points for a single event in a given crystal. The above plots are for different EmcGHit energy ranges. The Gaussian parameters $p 1$ and $p 2$ correspond to the $y$ and $x$ values at the maximum.

\section{Filtered time information}

The precision with which the time of the event could be measured was then investigated. As the aim of the EMC is to make precise measurements of the energy, any improvement in the resolution of the event time that can be obtained should not be at the expense of the energy resolution. However, an accurate measurement of the time may allow the rejection of backgrounds from hits at different times. For the polynomial fit case, the time is taken to correspond to the peak of the fit (Appendix A). To study the resolution, the difference between the measured time and the real time were plotted before and after the application of the polynomial fit. 

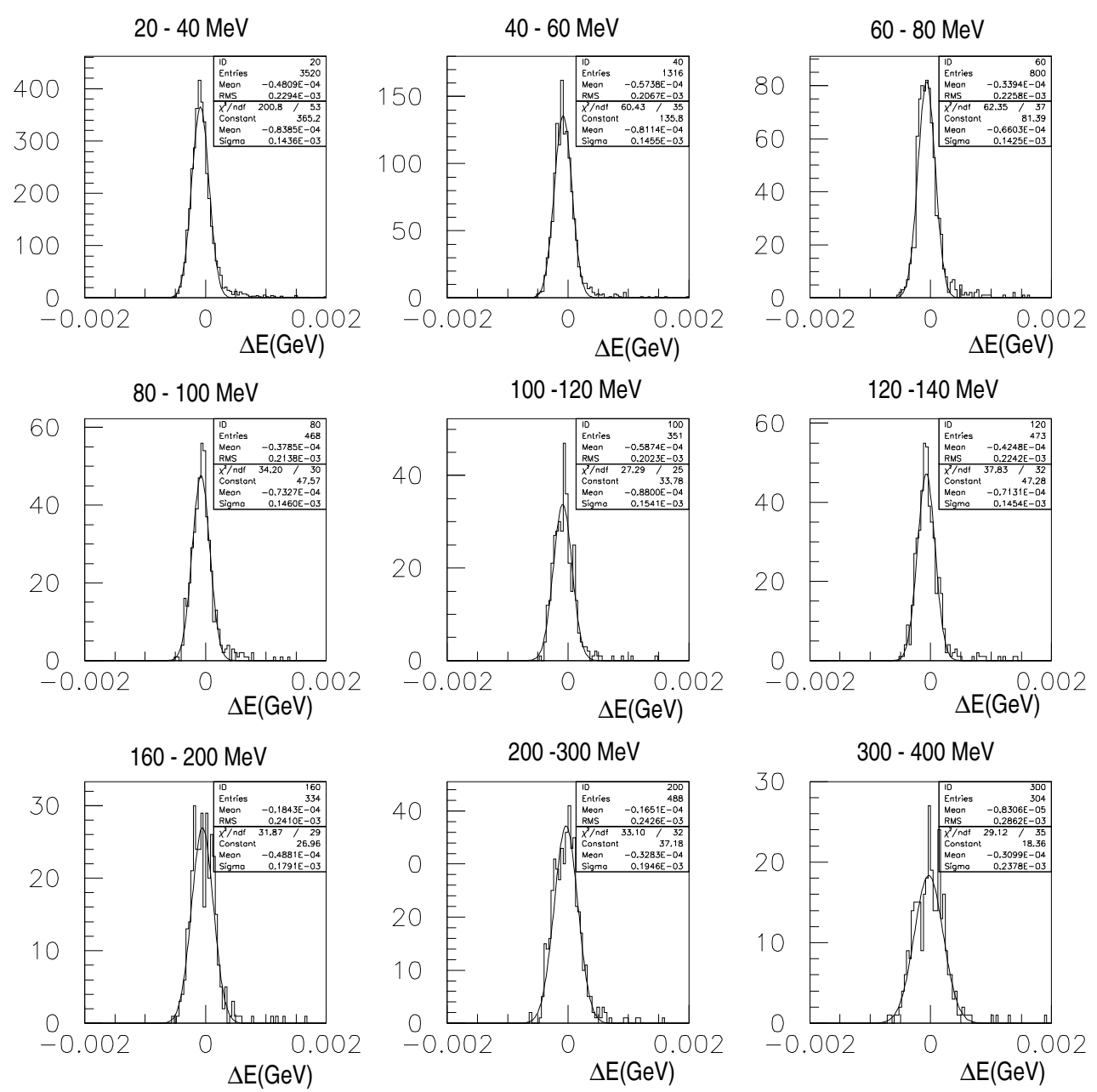

Figure 3.15: The difference between the EmcGHit energy and the fitted digi energy for different energy ranges. Thy y axis for all the plots is in events/0.04 Gev. The fit gives an overall improvement in the energy resolution of about $3-50 \%$ over the normal case, fig 3.13 .

This was done for various energy ranges so that any energy dependent variation of the time resolution could be investigated. As expected, no such variation was seen. A resolution plot for the $20 \mathrm{MeV}$ to $100 \mathrm{MeV}$ energy range can be seen in figure 3.17. Here, the top and bottom plots showing the resolutions before and after the fit respectively.

The reason for the shapes of the timing distributions is as follows. The waveform time bins are of integer value and have a width of $269 \mathrm{~ns}$. Therefore, before the application of the fit, an error of up to about $134 \mathrm{~ns}$ (the time corresponding to half a bin) is always induced on any time measurement made. This can be seen in the 


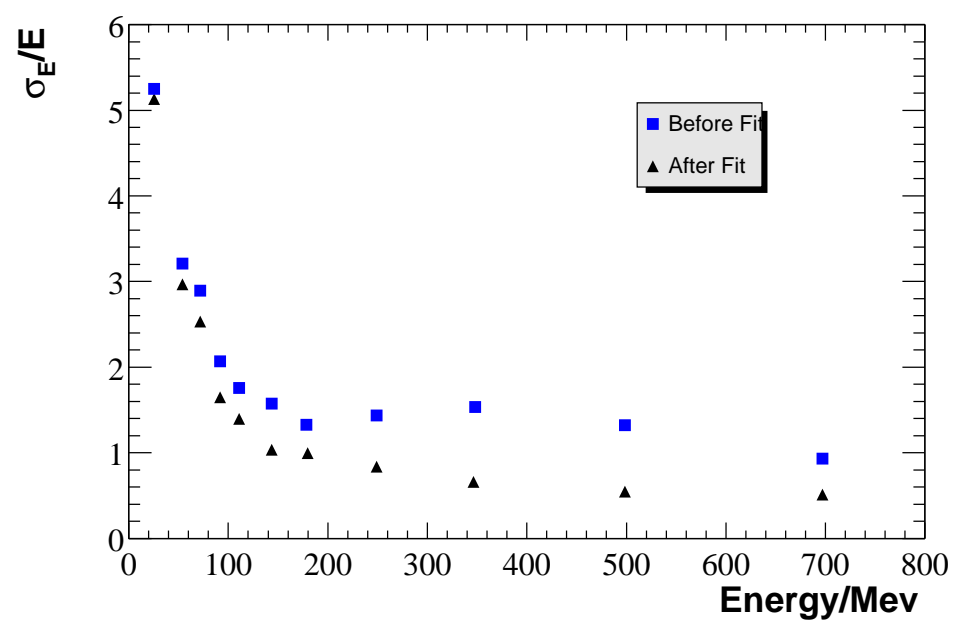

Figure 3.16: Energy resolutions as a function of energy before (squares) and after (triangles) the polynomial fit. An improvement in the resolution of about $3 \%$ for $30 \mathrm{MeV}$ photons and of about $40 \%$ for $700 \mathrm{MeV}$ photons is observed.

top plot of figure 3.17 where the distribution is from about -133 ns to about $+133 \mathrm{~ns}$ with an an RMS of about 80 ns. This is in good agreement with the expected RMS of $269 / \sqrt{12}=77.7 \mathrm{~ns}$. The application of the polynomial fit removes this quantisation of the bins and time is no longer measured in units of 269 ns. This is, of course, as a result of the fit returning a maximum within a given bin. The timing resolution in this case (figure 3.17b) therefore has a Gaussian shape rather than the top hat shape seen before. This gives rise to an improvement in the time resolution of about $90 \%$.

\subsection{Chapter summary}

An overview of the $B A B A R$ data acquisition system and the calorimeter front end electronics was given. The readout modules utilized by the calorimeter to gather and process the data have been described. An overview of the simulation software used by $B A B A R$ was given. The calorimeter front end electronics simulation software was then described. This included a discussion of the background noise mixing algorithms and the matched digital filter algorithms.

Two forms of background mixing processes were described. The first method, GHit mixing, used MC generated background samples. The other, event mixing, 

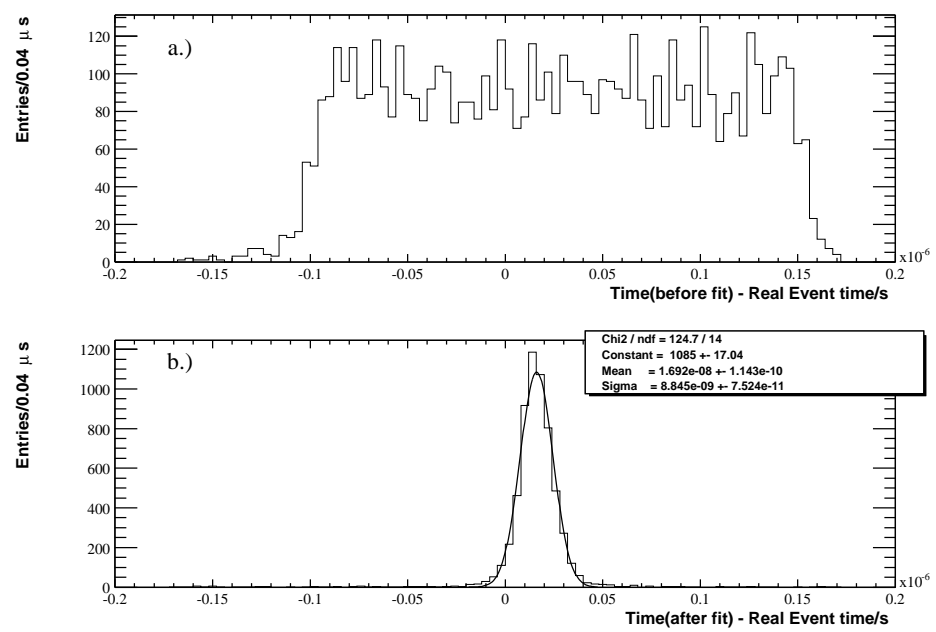

Figure 3.17: The difference between the digi time and the real event time before and after the polynomial fit. The fit gives a $90 \%$ improvement in the resolution over the normal case.

had the functionality to use both simulated and real backgrounds from random trigger events. A comparison between the two methods using a simulated background sample was presented. After an overview of the matched digital filter algorithms, a polynomial fit that was used to improvement the observed resolution was presented. It was concluded that using the fit gave an energy dependent improvement in the energy resolution of $4-40 \%$ and an improvement of about $90 \%$ in the timing resolution. 


\section{Chapter 4}

\section{Dilepton analysis and event selection}

\subsection{Introduction}

The current theoretical and experimental status of $\Delta m_{d}$ and the motivation for the measurement was given in chapter 1. The dilepton tagging performed at BABAR is expected to be more efficient than the semi-exclusive tagging performed for the time integrated measurements at the ARGUS [57] and CLEO [58] experiments.

An overview of the dilepton analysis as used by the BABAR experiment will be given in this chapter. In particular, a discussion of the event selection used to isolate the dilepton events will be presented. This will include an overview of the lepton identification used by the BABAR experiment with a discussion of the efficiencies and purities obtained. An overview of the background sources expected in this analysis and the selection cuts that were used to reduce the contamination from such backgrounds will then be presented. The chapter will end with a summary of the selected data and MC sample. A comprehensive discussion of the fitting procedure, the extraction of $\Delta m_{d}$ and systematic effects will be presented in chapter 5 .

\subsection{Dilepton analysis method}

The mixing parameter, $\Delta m_{d}$, can be extracted by comparing the rates of mixed $\left(B^{0} B^{0}, \bar{B}^{0} \bar{B}^{0}\right)$ and unmixed $\left(B^{0} \bar{B}^{0}\right)$ B meson pair decays in a time dependent asymmetry. The decay rates for mixed $\left(N_{\text {mixed }}\right)$ and unmixed $\left(N_{\text {unmixed }}\right)$ events are given by, 


$$
\begin{aligned}
N_{\text {mixed }} & \propto e^{-\Delta t / \tau_{B^{0}}}\left[1-\cos \left(\Delta m_{d} \Delta t\right)\right] \\
N_{\text {unmixed }} & \propto e^{-\Delta t / \tau_{B^{0}}}\left[1+\cos \left(\Delta m_{d} \Delta t\right)\right]
\end{aligned}
$$

where $\tau_{B^{0}}$ is the neutral B lifetime. The asymmetry can thus be written as,

$$
A_{\Delta m_{d}}=\frac{N_{\text {unmixed }}-N_{\text {mixed }}}{N_{\text {unmixed }}+N_{\text {mixed }}}=\cos \left(\Delta m_{d} \Delta t\right)
$$

The time, $\Delta t$, in this case is the proper time between the decays of the two $\mathrm{B}$ mesons. This is a direct result of the coherent nature of the $\Upsilon(4 \mathrm{~S})$ as described in section 1.3.2. Therefore, until the decay of one of the B mesons, there will be exactly one $B^{0}$ and one $\bar{B}^{0}$. Following the subsequent decay of one of the $\mathrm{B}$ mesons, the other will undergo mixing until its decay. The knowledge of the exact $\Upsilon(4 \mathrm{~S})$ vertex is thus not required for the measurement of $\Delta m_{d}$ and only the $\mathrm{B}$ decay vertices need to be calculated. The method used to do this will be presented in section 5.2.

The flavour of the decaying $\mathrm{B}$ meson can be determined by using leptons from semileptonic B decays as the tagging particles. In this case, the sign of the charge of the lepton is equal to the sign of the charge of the b quark within the B meson. The semileptonic decay of a $B^{0}$ thus yields a positively charged lepton $\left(l^{+}\right)$whilst that of a $\bar{B}^{0}$ gives a negatively charged $\left(l^{-}\right)$one. The events considered in this analysis, dilepton events, are ones in which both B mesons decay semileptonically. The semileptonic (electron or muon) branching ration for B mesons is about $20 \%$. The event sample used in this analysis thus represents $4 \%$ of the $\Upsilon(4 \mathrm{~S}) \rightarrow B \bar{B}$ decays. The total number of same sign dileptons constitutes the mixed category and the total number of opposite sign dileptons represents the unmixed category.

$$
\begin{aligned}
N_{\text {unmixed }} & =N\left(l^{+}, l^{-}\right) \\
N_{\text {mixed }} & =N\left(l^{+}, l^{+}\right)+N\left(l^{-}, l^{-}\right)
\end{aligned}
$$

It is therefore possible to express the asymmetry, $A_{\Delta m_{d}}(|\Delta t|)$, in terms of the number of same sign and opposite sign dilepton events as, 


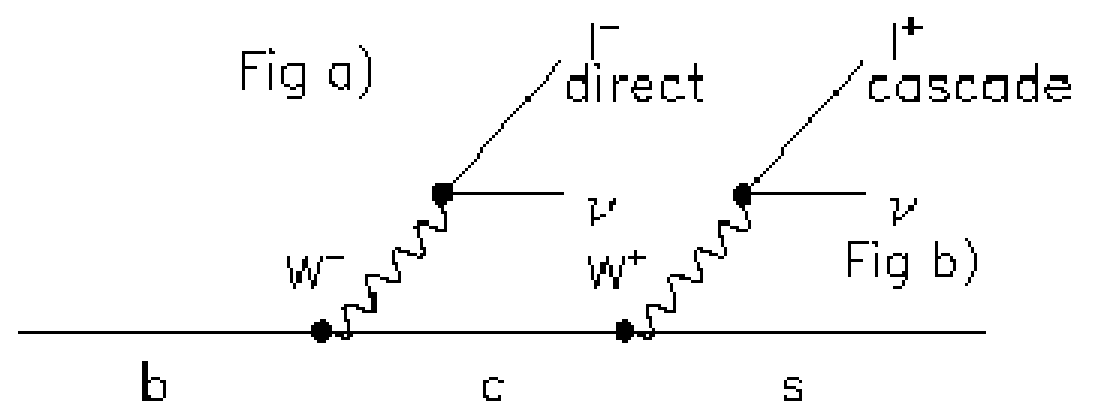

Figure 4.1: The semileptonic decays of the bottom quark (figure a) into a charm quark and a lepton (direct lepton). Figure $b$ shows the semileptonic decay of the charm quark into a strange and a lepton (cascade lepton)

$$
A_{\Delta m_{d}}(|\Delta t|)=\frac{N\left(l^{+}, l^{-}\right)-\left[N\left(l^{+}, l^{+}\right)+N\left(l^{-}, l^{-}\right)\right]}{N\left(l^{+}, l^{-}\right)+\left[N\left(l^{+}, l^{+}\right)+N\left(l^{-}, l^{-}\right)\right]}
$$

A lepton arising from the semileptonic decay of the $\mathrm{B}$ is referred to as a primary (direct) lepton, figure 4.1a. As seen in figure 4.1b, it is also possible for the charm to decay semileptonically. The lepton from such a decay is referred to as a cascade (secondary) lepton. If correctly identified, these leptons can also be used to tag the flavour of the B. But, due to the additional charm lifetime and the resulting poor $\mathrm{z}$ resolution, these were not used for tagging purposes in this analysis. Cascades are thus a source of background and a discussion on this can be found in section 4.7.1.

\subsection{Event sample}

The data sample used in this analysis was collected by the BABAR detector from January to June 2000. This corresponds to an integrated luminosity of $7.72 \mathrm{fb}^{-1}$ of data taken on the $\Upsilon(4 \mathrm{~S})$ resonance (on-resonance) and $1.18 \mathrm{fb}^{-1}$ of data taken at $40 \mathrm{MeV}$ below the resonance (off-resonance). This data sample ("Osaka sample") was used for the BABAR results presented at the ICHEP 2000 Osaka conference. The off-resonance data was not used in the extraction of $\Delta m_{d}$ but was used to validate the background characterisations described in section 5.5.3. The MC sample used consisted of signal dilepton, generic $B^{0} \bar{B}^{0}$ decays, generic $B^{+} B^{-}$decays, and 


\begin{tabular}{|c|c|}
\hline Event Type & $\mathcal{L}\left(\mathrm{fb}^{-1}\right)$ \\
\hline \hline Signal MC & 1.14 \\
$B^{0} \bar{B}^{0} \mathrm{MC}$ & 4.32 \\
$B^{+} B^{-} \mathrm{MC}$ & 4.52 \\
$c \bar{c} \mathrm{MC}$ & 2.68 \\
$u \bar{u}, s \bar{s}, d \bar{d} \mathrm{MC}$ & 1.72 \\
\hline On-peak data & 7.72 \\
Off-peak data & 1.18 \\
\hline
\end{tabular}

Table 4.1: Event Sample: Summary of the MC and data sample used in this analysis.

continuum $c \bar{c}$ and $u \bar{u}, s \bar{s}, d \bar{d}$ events. A summary of the relevant luminosities is given in table 4.1 .

The data sample was divided into six on-resonance and five off-resonance subsamples corresponding to different data taking time periods and hence different conditions. A luminosity summary of the sub-samples is given in table 4.2.

\begin{tabular}{|c|c|c|}
\hline Sample Number & On-resonance $\mathcal{L}\left(\mathrm{fb}^{-1}\right)$ & Off-resonance $\mathcal{L}\left(\mathrm{fb}^{-1}\right)$ \\
\hline \hline Sample 1 (Osaka 1) & 0.24 & 0.01 \\
Sample 2 (Osaka 2) & 0.53 & 0.14 \\
Sample 3 (Osaka 3) & 2.27 & 0.28 \\
Sample 4 (Osaka 4) & 1.55 & 0.30 \\
Sample 5 (Osaka 5) & 1.80 & 0.00 \\
Sample 6 (Osaka 6) & 1.33 & 0.45 \\
\hline Total & 7.72 & 1.18 \\
\hline
\end{tabular}

Table 4.2: On-resonance and off-resonance data sub-samples. These correspond to different data taking time periods between January and June 2000.

\subsection{Multi-Hadron Selection}

The data sample used in this analysis was required to pass the BABAR multi-hadron selection procedure [30]. For an event to be classified as a multi-hadronic event, the following criteria must be satisfied:

- The event should contain a minimum of four charged tracks with at least three of the tracks registering hits in the $\mathrm{DCH}$. 
- The primary vertex is required to lie within $5 \mathrm{~mm}$ in $\mathrm{x}$ and $\mathrm{y}$ of the nominal beam spot position

- The normalised second Fox-Wolfram moment (equation 4.13) is required to be less than 0.7 .

- The total energy associated with the event is required to be greater than $5 \mathrm{GeV}$.

The above cuts yield a selection efficiency of about $96 \%$ for $B \bar{B}$ MC events.

\subsection{Track quality cuts}

Since the measurement of $\Delta m_{d}$ involves a position measurement, namely the B decay vertex, only tracks that are well reconstructed were used in this analysis. In selecting these tracks, the following cuts were used.

- Distance of Closest Approach of the track (DOCA) : The DOCA of the track $\left(d_{0}\right)$ to the beam-spot was required to be less than $1 \mathrm{~cm}$.

- Position of Closest Approach (POCA) : The z coordinate of the POCA of the track to the beam-spot $\left(z_{0}\right)$ was required to be less than $6 \mathrm{~cm}$.

- Number of hits in the drift chamber $\left(N_{D C H}\right)$ was required to be greater than 20.

- At least two $z$-layer silicon vertex tracker hits $\left(N_{S V T}\right)$ were required. A more detailed discussion on this cut along with the efficiency obtained can be found in section 4.8 .

The combined selection efficiency for the DOCA, POCA and the drift chamber hits cuts was found to be more than $93 \%$ for signal MC. 


\subsection{Lepton identification}

As leptons are used for tagging purposes, the correct identification of leptons is crucial to the success of this analysis. As a very pure sample of leptons are required, the official BABAR very tight electron [61] and muon [62] selectors were used. These selectors are optimised to deliver a sample of leptons with a very high purity. This is, of course, at the expense of the overall efficiency. But, it should be noted that this loss in efficiency is more than compensated by the gain obtained in the purity. The selection cuts used by the individual selectors will now be presented.

\subsubsection{Electron identification}

The electron identification algorithms use the information from the EMC, DCH and the DIRC. The ratio of the EMC shower energy to the track momentum $(\mathrm{E} / \mathrm{p})$ was found to provide the best discrimination between electrons and other charged particle species. It was possible to enhance the selection performance by also considering other variables. The following cuts were then used for electron identification.
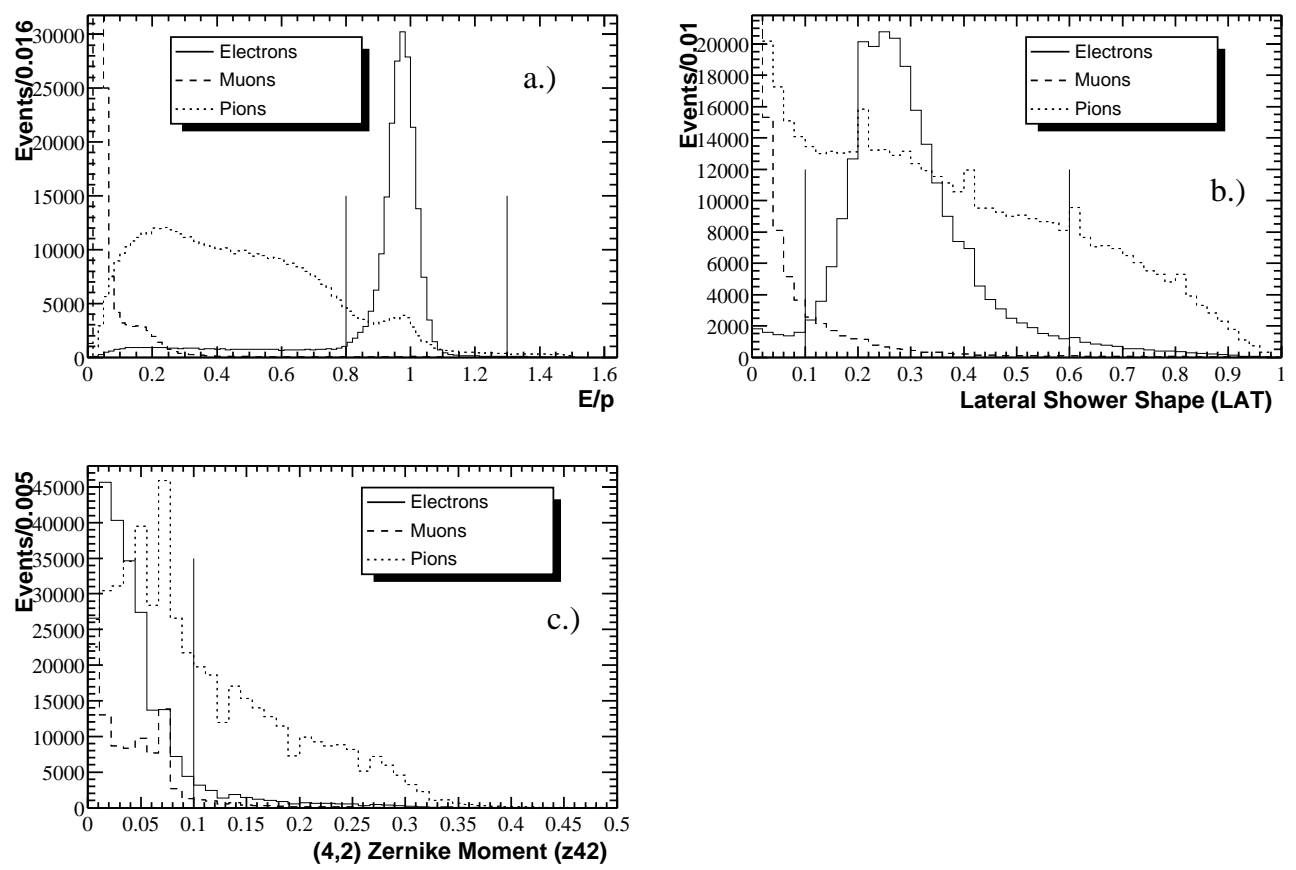

Figure 4.2: Electron selector : Electron, muon and pion data control sample comparison plots for $E / p$ (figure a), lateral moment (LAT) (figure b) and the 4,2 Zernike moment (Z42) (figure c). 


\section{$\mathrm{E} / \mathrm{p}$}

As previously stated, this provides the best discriminating power. As electrons lose almost all of their energy in the EMC, for an ideal calorimeter an E/p of one is expected. But in reality, due to bremsstrahlung, shower leakage and resolution effects, the $\mathrm{E} / \mathrm{p}$ for electrons is different from unity. Charged hadrons such as pions and kaons either pass through the EMC without any interactions or deposit a large fraction of their energy (figure 4.2a). But, nevertheless, they rarely lose all of their energy in the EMC and thus an E/p that is less than one is expected. A cut on the $\mathrm{E} / \mathrm{p}$ of $0.8<E / p<1.3$ was therefore used.

\section{Lateral EMC shower shape (LAT)}

It is possible to increase the electron-hadron discrimination obtained from E/p measurements by considering the shapes of the showers produced. The lateral shower distributions from hadronic showers differ significantly from those from electromagnetic showers. If the number of crystals associated with a shower is given by $\mathrm{N}$, the LAT of a shower is defined as [59],

$$
L A T=\frac{\sum_{i=3}^{N} E_{i} r_{i}^{2}}{\sum_{i=3}^{N} E_{i} r_{i}^{2}+E_{1} R_{0}^{2}+E_{2} R_{0}^{2}}
$$

Here, the energy deposits are labeled such that $E 1 \geq E_{2} \geq \ldots . \geq E_{N}, R_{0}$ is the average distance between crystals $(=5 \mathrm{~cm}$ at $B A B A R)$ and $r_{i}$ is the distance from the centre of the cluster to the centre of the $i^{\text {th }}$ crystal. For electrons, a low LAT values is expected, figure $4.2 \mathrm{~b}$. In this analysis, a cut on the LAT variable of $0.1<L A T<0.6$ was used.

\section{Zernike moment}

Zernike moments were first introduced at ZEUS in 1996 [60]. The idea is to expand the energy distributions into various moments using a complete and orthogonal set of functions. In constructing the Zernike moments, the energy distribution per cluster is expanded using the Zernike functions. Given a polynomial $f_{n m}$ such that,

$$
f_{n m}(r)=\sum_{s=0}^{(n-m) / 2} \frac{(-1)^{s}(n-s) ! r^{n-2 s}}{s !((n+m) / 2-s) !((n-m) / 2-s) !}
$$


the Zernike function is defined as,

$$
Z_{n m}(r, \phi)=f_{n m}(r) e^{-i m \phi}
$$

such that, for the integers $n, m \geq 0, n-m$ is even and $m \leq n$. The Zernike moments, $A_{n m}$, are then defined as,

$$
\begin{aligned}
& a_{n m}=\sum_{r_{i} \leq R_{0}}=\frac{E_{i}}{E} Z_{n m}\left(\frac{r_{i}}{R_{0}}, \phi_{i}\right) \\
& A_{n m}=\left|a_{n m}\right|
\end{aligned}
$$

At $B A B A R, R_{0}=15 \mathrm{~cm}$. In this analysis a cut on the 42 Zernike moment, $A_{42}$, of $A_{42}<0.1$ (figure $4.2 \mathrm{c}$ ) was used.

\section{$\mathrm{d} E / \mathrm{d} x$ measurement}

A cut in the $\mathrm{d} E / \mathrm{d} x$ measured by the DCH was also used by the electron selector. The $\mathrm{d} E / \mathrm{d} x$ of the track was required to be $550<\mathrm{d} E / \mathrm{d} x<850$ (in arbitrary units). The $\mathrm{d} E / \mathrm{d} x$ distributions for various track species were shown in figure 2.7 in chapter 2.

\section{Čerenkov angle}

A three sigma cut around the expected Čerenkov angle produced in the DIRC was used. The main motivation for this was the reduction of any residual kaon contamination.

\subsubsection{Electron identifier performance}

The performance of the electron identifier can be seen in figure 4.3 for data control samples. The electron control sample was made from Bhabha, gamma conversion and $e^{+} e^{-} \rightarrow e^{+} e^{-} e^{+} e^{-}$events. The pion contamination was calculated from using pions from $K_{S}^{0}$ decays. A detailed discussion on the control samples can be found at [61]. The selection efficiency and pion misidentification as a function of the momentum in the lab frame are shown in figure 4.3a. Figure 4.3b shows the selector performance as a function of the polar angle, $\theta$. This gives an efficiency of about $90 \%$ 
over the $0.5 \mathrm{GeV} / \mathrm{c}$ to $3.5 \mathrm{GeV} / \mathrm{c}$ momentum range. The efficiencies of electron, muon and pion selection over the kinematic region of interest in this analysis $(0.7 \mathrm{GeV} / \mathrm{c}$ $\left.<p_{l a b}<3.5 \mathrm{GeV} / \mathrm{c}\right)$ is given in table 4.3. These were calculated using the data control samples defined above and an electron identification efficiency of more than $80 \%$ and a pion mis-identification of about $3 \%$ is obtained.
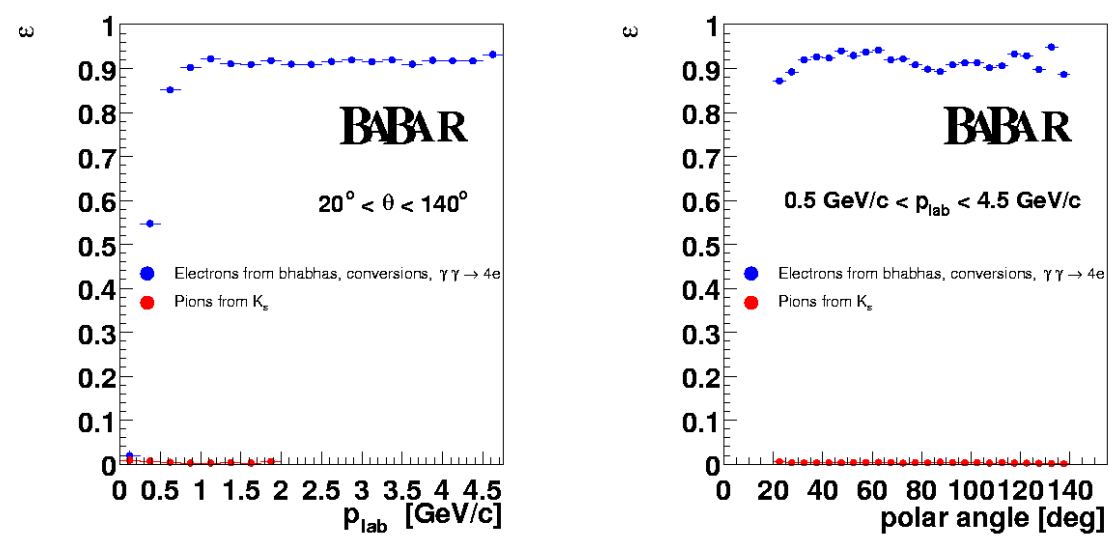

Figure 4.3: Electron selector performance as a function of momentum of the track in the lab frame and $\theta$ for electrons and pions from data control samples.

\begin{tabular}{|c|c|}
\hline Particle Type & $\mathcal{E}(\%)$ \\
\hline \hline $\mathrm{e}$ & 80.2 \\
$\pi$ & 2.9 \\
$\mu$ & 0.5 \\
\hline
\end{tabular}

Table 4.3: Electron selector efficiency and pion/muon misidentification efficiencies for the momentum region of interest in this analysis.

\subsubsection{Muon identification}

The IFR is the primary detector element used for the identification of muons. It is possible to enhance the IFR based muon selector by using information from the EMC. The muon selection algorithms used in this analysis do just that. For a track to be identified as a muon, the following selection criteria must be satisfied [62]. 

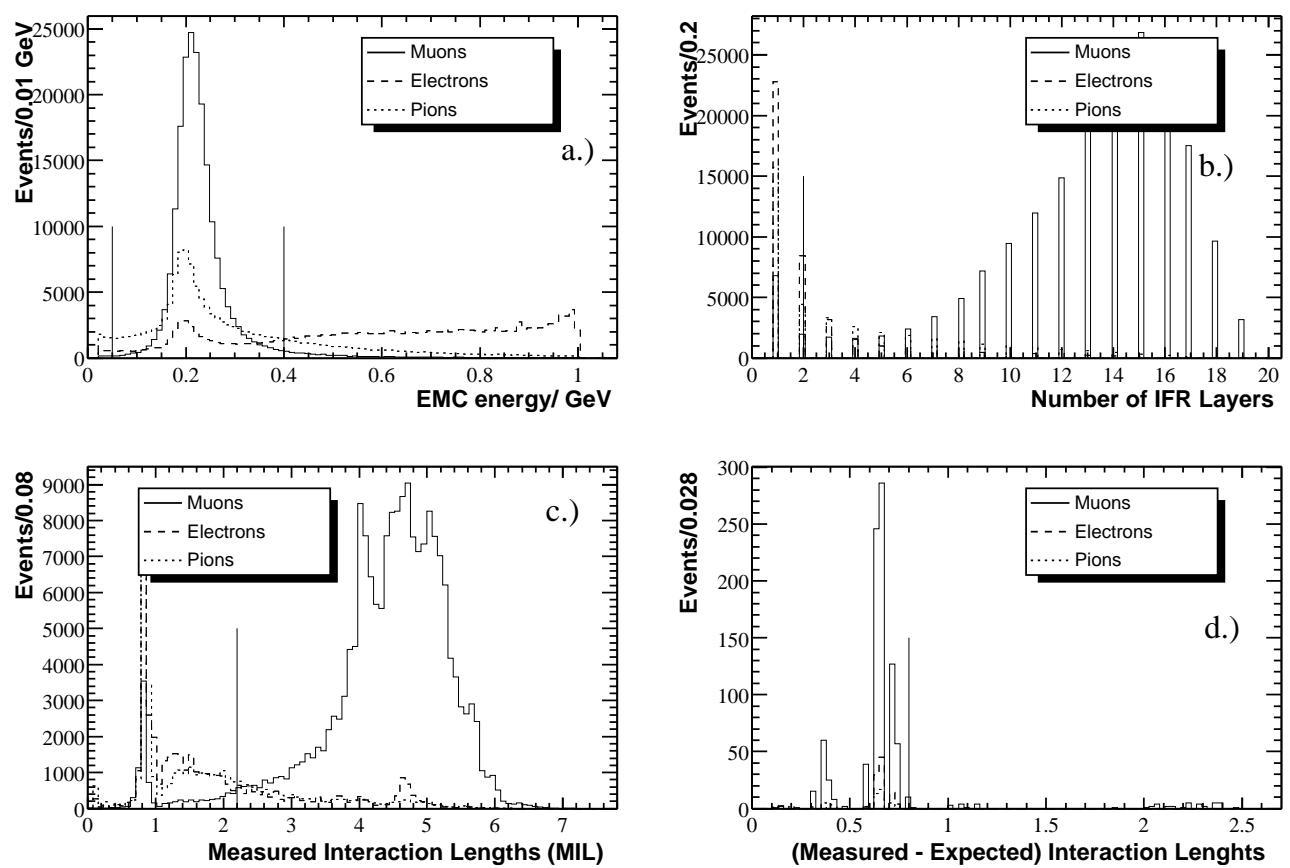

Figure 4.4: Muon selector : Muon, electron and pion data control sample comparison plots for the energy deposit in the EMC (figure a), Number of IFR layer hits (figure b), measured interaction lengths in the detector (figure $c$ ) and the deviation of the measured interaction lengths from the expected (figure d).

\section{EMC energy $\left(E_{E M C}\right)$}

Muons interact very weakly in the EMC and only lose a very small fraction of their energy, figure $4.4 \mathrm{a}$. Thus a cut on the EMC energy corresponding to a minimum ionizing peak of $0.05<E_{E M C}<0.4 \mathrm{GeV}$ was used.

\section{IFR layers $\left(N_{L}\right)$}

The selection requires at least two IFR layers per cluster, figure 4.4b.

\section{Interaction lengths in the IFR}

A rather important variable used in muon identification is the number of interaction lengths traversed by a track in the $B A B A R$ detector $(\lambda)$. A cut on $\lambda$ of $\lambda>2.2$ was required (figure 4.4c). This variable can be compared with the interaction lengths expected for a muon $\left(\lambda_{\text {expected }}\right)$. The deviation from this value is defined as (figure $4.4 \mathrm{~d})$, 


$$
\Delta \lambda=\lambda-\lambda_{\text {expected }}
$$

A cut on $\Delta \lambda$ of $\Delta \lambda<0.8$ was also required in the selection.

\section{Tracking $\chi^{2} /$ d.o.f}

In order to reduce the contamination from poorly reconstructed tracks, a cut on the $\chi^{2} /$ d.o.f $\left(\chi_{\text {trk }}^{2}\right)$ match between the extrapolated track from the drift chamber to the strips in the IFR was used. It was required that $\chi_{t r k}^{2}$ was less than five $\left(\chi_{t r k}^{2}<5\right)$.

\section{Cluster $\chi^{2} /$ d.o.f}

A cut on the $\chi^{2} /$ d.o.f of a third order polynomial [62] to the shape of the IFR cluster $\left(\chi_{f i t}^{2}\right)$ was also used. This was required to be less than three $\left(\chi_{f i t}^{2}<3\right)$.

\section{Track continuity $\left(T_{c}\right)$}

The track continuity variable is defined as,

$$
T_{c}=\left\{\begin{array}{c}
\frac{N_{L}}{L_{h}-F_{h}+1}: \text { if there is NO hit in the inner RPC layer } \\
\frac{N_{L}}{L_{h}-F_{h}}: \text { if there is a hit in the inner RPC layer }
\end{array}\right.
$$

where, as defined before, $N_{L}$ is the number of IFR layers in the cluster and $F_{h}$ and $L_{h}$ are respectively the numbers of the first and last IFR layers in the cluster. A description of the IFR layer numbering can be found in section 2.3.6. For an ideal detector, the track continuity distribution should be peaked around unity. A cut on $T_{c}$ of $T_{c}>0.34$ was used in this analysis.

\section{IFR strips}

A further cut on the average number of IFR strips $(\bar{m})$ and its standard deviation $\left(\sigma_{m}\right)$ was used. The standard deviation was required to satisfy $\sigma_{m}<4$ and the mean was required to satisfy $\bar{m}<8$. 


\subsubsection{Muon identifier performance}

The performance of the muon selector algorithm described above can be seen in figure 4.5. As in the case of the electron selector, these plots are also for data control samples. The muons used in this case were from $e^{+} e^{-} \rightarrow \mu^{+} \mu^{-} \gamma$ and $e^{+} e^{-}$ $\rightarrow e^{+} e^{-} \mu^{+} \mu^{-}$events. The pions used in the calculation of the mis-id were from $K_{S}^{0} \rightarrow \pi^{+} \pi^{-}$and $e^{+} e^{-} \rightarrow \tau^{+} \tau^{-}$events that decay with a 1-3 prong signature where one tau decays to a lepton and the other to three pions. The plots show the muon selection efficiency and pion mis-identification as a function of the lab momentum and the polar angle, $\theta$. An average muon selection efficiency of $70 \%$ with a pion mis-identification of $2.4 \%$ is obtained. The selection efficiencies integrated over the entire polar angle for the kinematic region of interest in this analysis is given in table 4.4. In this case a selection efficiency of more that $60 \%$ can be expected. The obtained pion mis-identification is about $3.7 \%$.
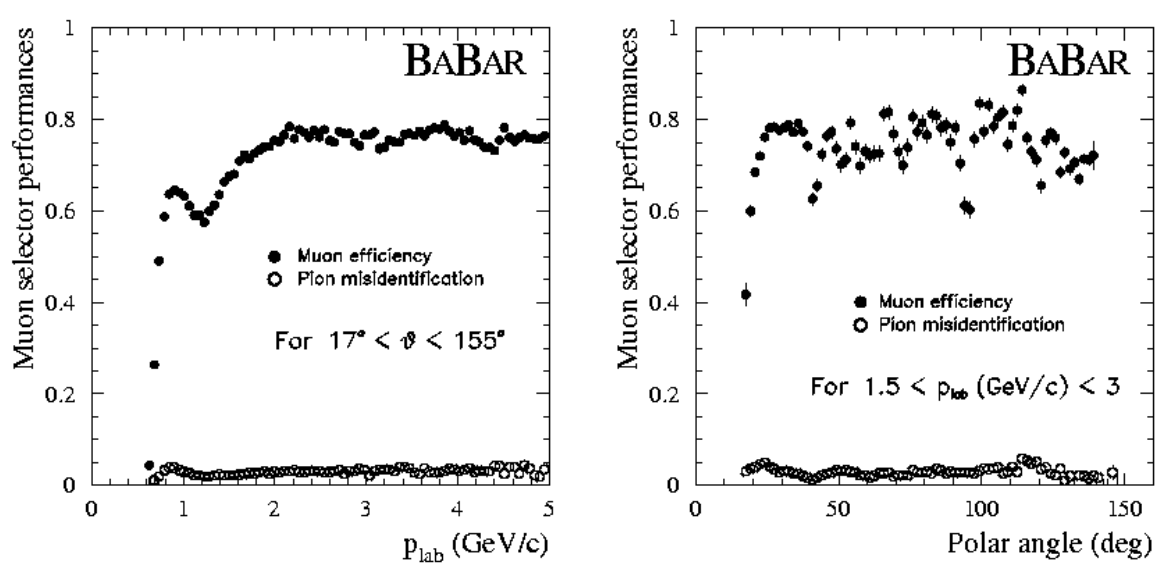

Figure 4.5: Muon selector efficiency as a function of the momentum of track in the lab frame and $\theta$.

\begin{tabular}{|c|c|}
\hline Particle Type & $\mathcal{E}(\%)$ \\
\hline \hline$\mu$ & 61.3 \\
$\pi$ & 3.7 \\
$\mathrm{e}$ & 0.4 \\
\hline
\end{tabular}

Table 4.4: Muon selector efficiency and pion/electron misidentification efficiencies for the momentum region of interest in this analysis. 


\subsection{Background reduction}

A discussion of the various sources of backgrounds that affected this measurement will now be presented. The main source of backgrounds was due to cascade leptons and this will be addressed first.

\subsubsection{Cascade leptons}

Two different ways in which cascades contribute to the analysis are shown in figure 4.6. Here, the semileptonic decay of the $\mathrm{b}$ quark is shown in figure 4.6a. The charm produced by this also decays semileptonically to a strange quark as shown in figure $4.6 \mathrm{~b}$. The cascade lepton thus produced has a charge opposite to that of the primary. The second way in which cascades can contribute is shown in figure 4.6c. Here, the $b$ undergoes a non-leptonic decay to a charm which then subsequently decay semileptonically. The cascade in this case will have the correct charge to tag the B flavour but due to the additional charm lifetime, will give the wrong vertex position. Cascades also contribute in one additional way. This is when a primary and a cascade are chosen from the same B meson.

It is not possible to discriminate between the primaries and the cascades by topological considerations alone. However, due to kinematic reasons, a cascade lepton is expected to have a $\Upsilon(4 \mathrm{~S})$ centre of mass (CMS) momentum $\left(p^{*}\right)$ that is less than that of a primary. A cut on the $p^{*}$ of the tracks was found to provide the best discrimination between primary and cascade leptons. The $p^{*}$ spectra for primaries and cascades are shown in figure 4.7. A lower cut on the $p^{*}$ of $1.2 \mathrm{GeV} / \mathrm{c}$ was therefore used. Furthermore, due kinematic reasons, the maximum $p^{*}$ for semileptonic decay is around $2.5 \mathrm{GeV} / \mathrm{c}$. A cut on $p^{*}$ of $1.2<p^{*}<2.5 \mathrm{GeV} / \mathrm{c}$ was thus used. With these cuts, a MC signal efficiency of about $62 \%$ was obtained (table 4.5 ). The motivation for such a high cut in $p^{*}$ which resulted in a significant loss of efficiency will be given in the following chapter.

The main method by which cascades contribute to the background is when a primary lepton is correctly identified and a cascade lepton is mis-identified as primary. In this case, the dominant contribution is from cascades due to the decay of the charged D mesons. For signal MC, the selection of a lepton from a neutral B and 

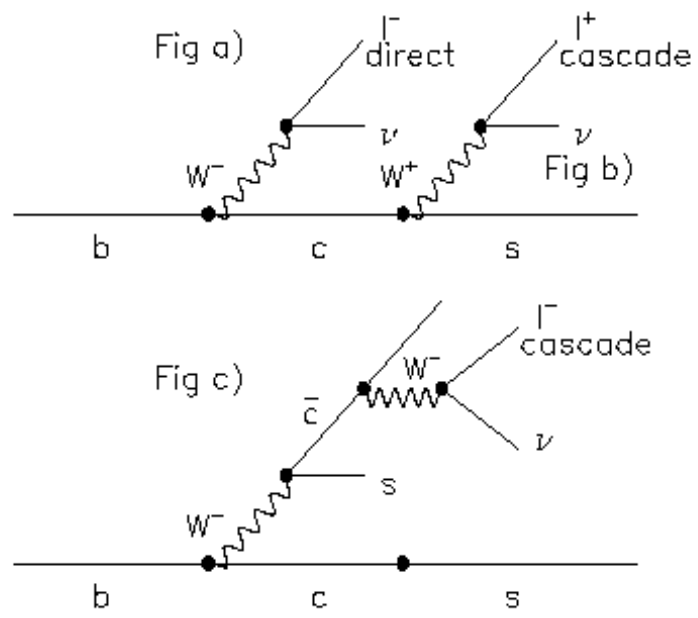

Figure 4.6: Semileptonic decay of the bottom quark (figure a) and that of the charm (figure b). The non-leptonic decay of the bottom quark to a charm quark is shown in figure c. Two types of cascades are produced; one with the correct tagging charge (figure $c$ ) and the other with the wrong tagging charge (figure b).

another from a charged D was found to have an efficiency of $10.7 \%$. Similarly, the selection of two leptons from a neutral B, neutral D combination was found to have an efficiency of about $5.4 \%$ for signal MC. The case where two cascade leptons were mis-identified as primary was found to be $0.5 \%$. The selection of two fake leptons or a cascade and one fake lepton were found to have efficiencies of $0.01 \%$ and $0.02 \%$ respectively. Also, the selection of one primary lepton and a fake lepton was found to have an efficiency of $0.9 \%$.

\subsubsection{Continuum backgrounds}

At the $\Upsilon(4 \mathrm{~S})$, the cross section for continuum production is greater than for $b \bar{b}$ (table 4.6). As a result a significant background from these continuum events can be expected.

In order to suppress these continuum background events, cuts on various event shape variables were considered. The ratio of the second to the zeroth Fox-Wolfram moment, figure 4.8, offered the best continuum reduction whilst keeping the signal loss to a minimum. The Fox-Wolfram moment $\left(R_{l}\right)$ is defined as, 


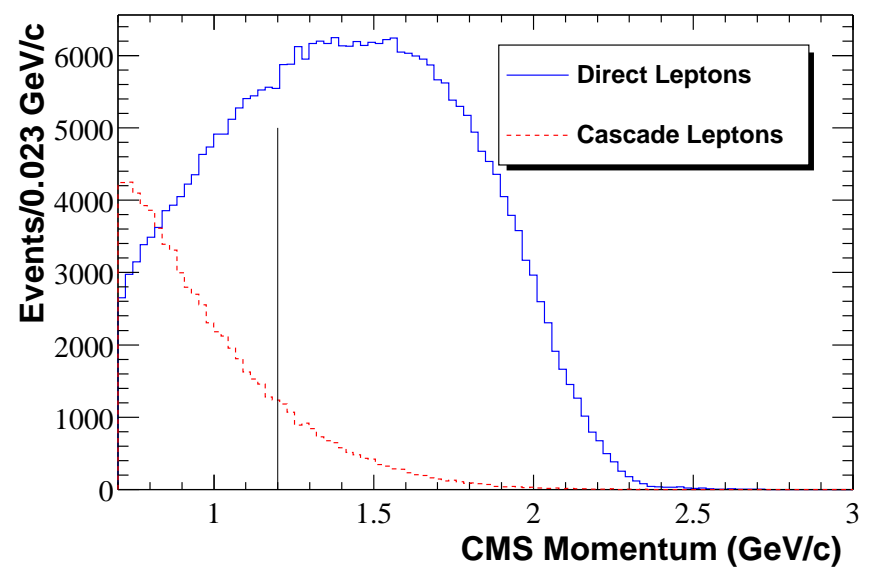

Figure 4.7: Primary and cascade lepton spectra in the CMS system. The momentum spectrum of the primary leptons is given by the solid histogram and that of the cascade leptons is given by the dashed histogram. The maximum expected CMS momentum is $2.5 \mathrm{GeV} / \mathrm{c}$. A lower cut of 1.2 $\mathrm{GeV} / \mathrm{c}$ is used.

\begin{tabular}{|c|c|}
\hline Event Type & $\mathcal{E}$ of $1.2<p^{*}<2.5 \mathrm{GeV} /$ c cut $(\%)$ \\
\hline \hline Signal MC & 61.6 \\
$B^{0} \bar{B}^{0} \mathrm{MC}$ & 43.2 \\
$B^{+} B^{-} \mathrm{MC}$ & 44.3 \\
$c \bar{c} \mathrm{MC}$ & 30.6 \\
$u \bar{u}, s \bar{s}, d \bar{d} \mathrm{MC}$ & 23.3 \\
\hline On peak data & 42.1 \\
Off peak data & 37.0 \\
\hline
\end{tabular}

Table 4.5: Selection efficiencies for the $1.2<p^{*}<2.5 \mathrm{GeV} / \mathrm{c}$ cut after the lepton identification and track quality cuts were applied. It should be noted that the generic B MC events also contain the signal events.

$$
R_{l}=\sum_{i, j} \frac{\left|\mathbf{p}_{\mathbf{i}}\right|\left|\mathbf{p}_{\mathbf{j}}\right|}{E_{v i s}^{2}} P_{l}(\cos (\theta))
$$

Here, $P_{l}$ are the Legendre polynomials, $E_{v i s}$ is the total visible energy, $\mathbf{p}_{\mathbf{i}, \mathbf{j}}$ are the particle momenta and $\theta_{i j}$ is the opening angle between particles $\mathrm{i}$ and $\mathrm{j}$. The zeroth order Legendre polynomial is given by

$$
P_{0}=\frac{1}{2}
$$

and the second order polynomial, $P_{2}$, is given by, 


\begin{tabular}{|c|c|}
\hline Event Type & Cross section (nb) \\
\hline \hline$b \bar{b}$ & 1.05 \\
$c \bar{c}$ & 1.30 \\
$u \bar{u}+d \bar{d}+s \bar{s}$ & 2.09 \\
\hline
\end{tabular}

Table 4.6: Cross sections at the $\Upsilon(4 \mathrm{~S})$.

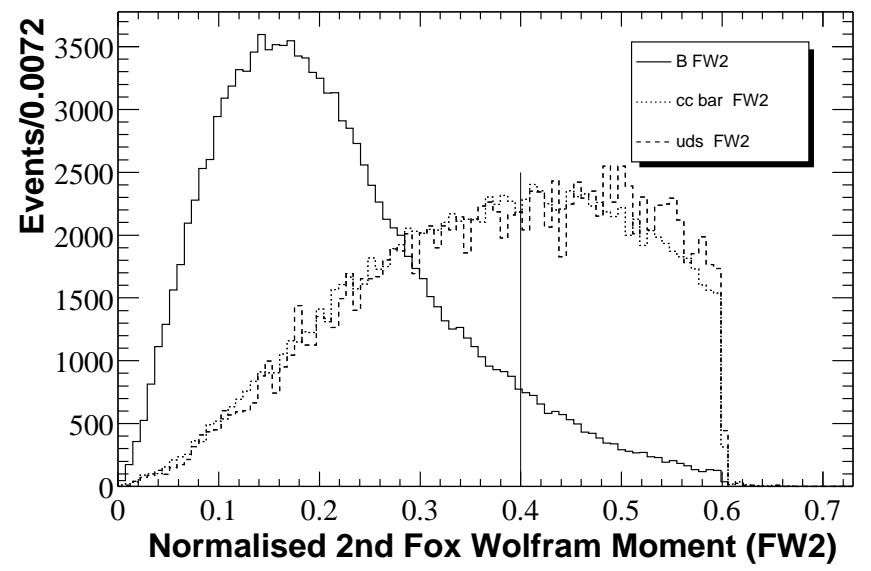

Figure 4.8: The normalised second Fox-Wolfram moment (FW2) distribution for $B^{0} \bar{B}^{0}$ and continuum $M C$ are shown. The $B^{0} \bar{B}^{0}$ distribution is given by the solid histogram and the continuum ones are given by the dashed histograms. A requirement on FW2 of FW2<0.4 was used.

$$
P_{2}=\frac{1}{2}\left(3 \cos ^{2} \theta-1\right)
$$

The normalised second Fox-Wolfram moment is then given by,

$$
F W 2=\frac{R_{2}}{R_{0}}=3 \sum_{i, j} \frac{\left|\mathbf{p}_{\mathbf{i}}\right|\left|\mathbf{p}_{\mathbf{j}}\right|}{E_{v i s}^{2}}\left[\cos ^{2}(\theta)-1\right]
$$

For this analysis, the normalised second Fox-Wolfram moment was required to be less than 0.4. The selection efficiencies for this cut for MC events and data are given in table 4.7. As seen, with the above cut, a reduction in the continuum background of about $45 \%$ and a signal efficiency of about $90 \%$ was obtained.

\subsection{3 $J / \psi$ backgrounds}

Due to the overlap in the lepton momentum spectra with leptons from semileptonic B decays, leptons from $J / \psi$ decays are a source of backgrounds in this analysis. The lepton CMS momentum spectra for these two cases are shown in figure 4.9. 


\begin{tabular}{|c|c|}
\hline Event Type & $\mathcal{E}$ of Fox-Wolfram cut $(\%)$ \\
\hline \hline Signal MC & 89.8 \\
$B^{0} \bar{B}^{0} \mathrm{MC}$ & 92.1 \\
$B^{+} B^{-} \mathrm{MC}$ & 92.4 \\
$c \bar{c} \mathrm{MC}$ & 54.8 \\
$u \bar{u}, s \bar{s}, d \bar{d} \mathrm{MC}$ & 52.3 \\
\hline On peak data & 77.6 \\
Off peak data & 56.6 \\
\hline
\end{tabular}

Table 4.7: The selection efficiencies for the cut on the normalised 2nd Fox-Wolfram moment after the application of the lepton identification and track quality cuts.

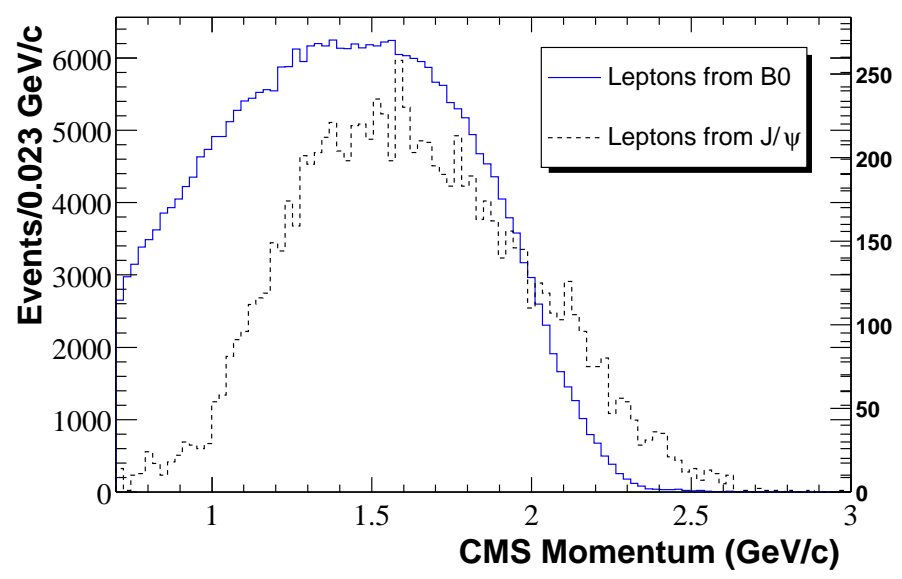

Figure 4.9: $M C$ Momentum spectra in the CMS frame for leptons from $B$ decays (solid line) and leptons from $J / \psi$ decays (dashed line).

A $J / \psi$ rejection that is based purely on momentum considerations is thus not feasible. The technique employed here involved the combination of all leptonic tracks in the event to construct the invariant mass. In doing this only opposite sign combinations were considered. The tracks that gave an invariant mass that fell within a cut window around the $J / \psi$ mass were rejected. In order to account for the radiative tail in the $J / \psi \rightarrow e^{+} e^{-}$distribution (figure 4.10b), two different cuts were employed. For muon pair events, a $60 \mathrm{MeV} / \mathrm{c}^{2}$ cut between $3.07 \mathrm{GeV} / \mathrm{c}^{2}$ and $3.13 \mathrm{GeV} / \mathrm{c}^{2}$ was used. For electron pair events, a much wider $180 \mathrm{MeV} / \mathrm{c}^{2}$ cut between $2.95 \mathrm{GeV} / \mathrm{c}^{2}$ and $3.13 \mathrm{GeV} / \mathrm{c}^{2}$ was used. With these cuts, a $J / \psi$ selection efficiency of $1.5 \%$ for muon pair events and an efficiency of $28.2 \%$ for electron pair events was obtained. The total signal lost due to the cuts was found to be $1.1 \%$ 
for the muon channel and $2.3 \%$ for the electron channel. The total proportion of leptons from $J / \psi$ decays in the final MC event sample was found to be about $0.8 \%$.
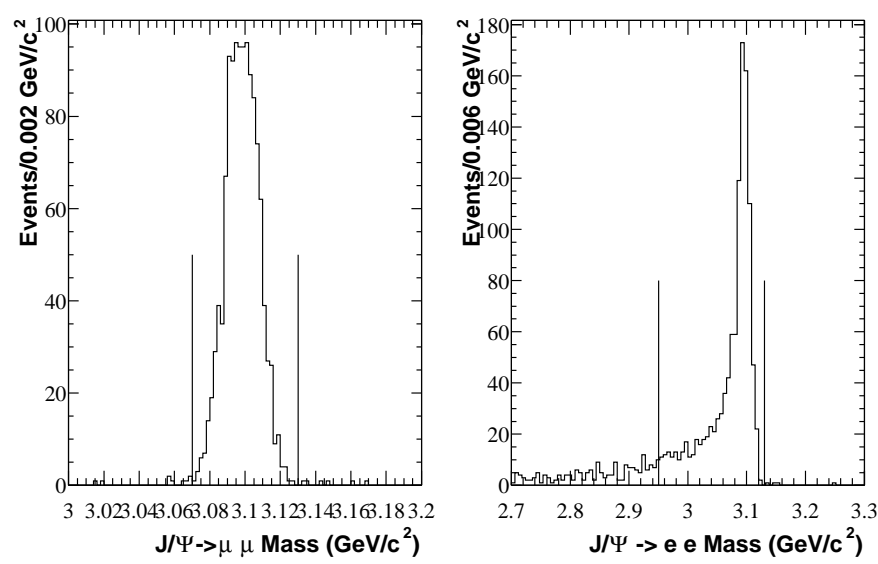

Figure 4.10: $M C J / \psi$ mass spectra for decays to muons (figure a) and electrons (figure $b$ ). $A$ $60 \mathrm{MeV} / \mathrm{c}^{2}$ cut around the $\mathrm{J} / \psi$ mass spectrum for the muon case was used. The radiative tail due to Bremsstrahlung can be seen in the electron plot, figure $b$. As a result, the lower bound on the cut window was reduced to $2.95 \mathrm{GeV} / \mathrm{c}^{2}$.

\subsubsection{Gamma conversion backgrounds}

Another source of backgrounds considered were electrons from gamma conversions. The contamination from these electrons can be reduced by employing the same technique used for $J / \psi$ decays. Once again, all opposite sign leptonic tracks were combined to form an invariant mass. The invariant mass spectrum for electron pairs from gamma conversions can be seen in figure 4.11. Conversion events are characterised by a very low invariant mass. Thus the invariant mass of the combination was required to be greater than $50 \mathrm{MeV} / \mathrm{c}$. With this cut, the selection efficiency for conversion events was found to be $15.6 \%$ whilst the signal lost was found to be negligible. The proportion of electrons from gamma conversions in the final MC sample was calculated to be around $0.7 \%$.

\subsubsection{Bhabha and $\gamma \gamma$ backgrounds}

Due to their very high cross section at the $\Upsilon(4 \mathrm{~S})$, Bhabhas and, in particular, radiative Bhabhas play an important role in the dilepton analysis. Bhabha events can 


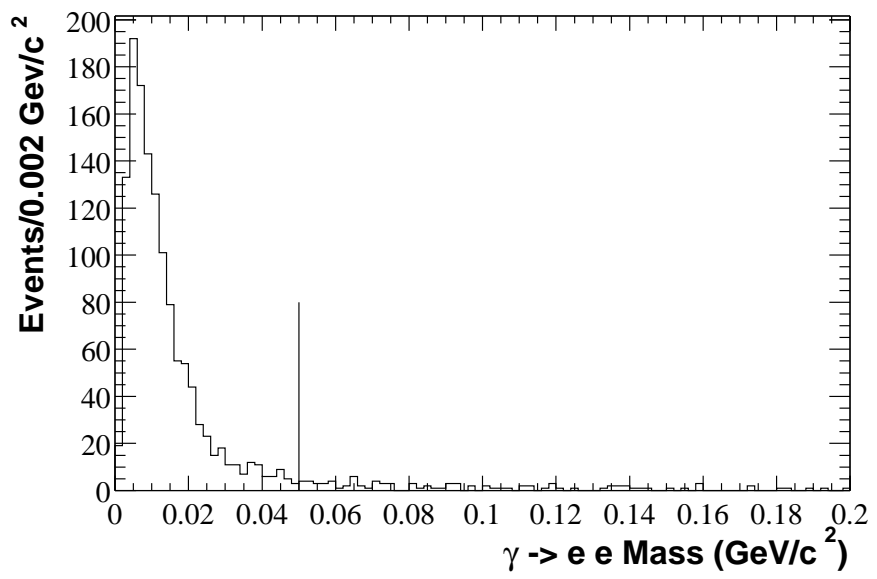

Figure 4.11: $M C$ invariant mass spectrum of electrons from gamma conversions. A cut in the invariant mass of greater than $50 \mathrm{MeV} / \mathrm{c}$ was used.

be suppressed by selecting events with at least five charged tracks. This cut alone, however, does not reduce the contamination from radiative Bhabhas sufficiently. A further cut on the aplanarity of the event was used to reduce the contamination from radiative Bhabha events. The aplanarity $(\mathrm{A})$, which is a measure of the transverse component of the momentum out of the event plane, is defined as,

$$
A=\frac{2}{3} \lambda_{3}
$$

where $\lambda_{3}$ is the smallest eigenvalue of the Sphericity tensor $\left(S^{\alpha \beta}\right)$. The tensor is given by

$$
S^{\alpha \beta}=\frac{\sum_{i} p_{i}^{\alpha} p_{i}^{\beta}}{\sum_{i}\left|\mathbf{p}_{\mathbf{i}}\right|^{2}}
$$

For completely planar events, an aplanarity of zero is obtained. For this analysis, the event aplanarity was required to be greater than 0.01 . The selection efficiencies for $\mathrm{MC}$ events with the above cut are shown in table 4.8. The efficiency for signal MC was found to be about $98 \%$ whilst the efficiencies for radiative Bhabhas and two photon events were $0.3 \%$ and $0.2 \%$ respectively.

\subsection{SVT z layer hits}

In order to remove poorly reconstructed tracks, a cut on the z layer hits in the SVT was employed. A definition of the SVT layers is given in section 2.3.2. Each track 


\begin{tabular}{|c|c|}
\hline Event Type & $\mathcal{E}$ of Aplanarity cut (\%) \\
\hline \hline Signal MC & 97.6 \\
$B^{0} \bar{B}^{0} \mathrm{MC}$ & 98.4 \\
$B^{+} B^{-} \mathrm{MC}$ & 98.6 \\
Radiative Bhabha MC & 0.3 \\
Two photon MC & 0.2 \\
\hline Bhabha + radiative Bhabha data & $<0.01$ \\
On peak data & 92.5 \\
Off peak data & 83.6 \\
\hline
\end{tabular}

Table 4.8: Selection efficiencies for the aplanarity cut after the application of the lepton identification and track quality cuts.

was required to have at least two SVT z hits, one in the first z layer and the other in either of layers two or three. With this cut, a selection efficiency of $82 \%$ for signal MC and $60 \%$ for on peak data was obtained. The efficiencies for other event types are shown in table 4.9 .

\begin{tabular}{|c|c|}
\hline Event Type & $\mathcal{E}$ of the SVT z hit cut $(\%)$ \\
\hline \hline Signal MC & 82.0 \\
$B^{0} \bar{B}^{0} \mathrm{MC}$ & 76.9 \\
$B^{+} B^{-} \mathrm{MC}$ & 75.3 \\
$c \bar{c} \mathrm{MC}$ & 55.3 \\
$u \bar{u}, s \bar{s}, d \bar{d} \mathrm{MC}$ & 26.6 \\
\hline On peak data & 60.1 \\
Off peak data & 39.8 \\
\hline
\end{tabular}

Table 4.9: Selection efficiencies for the SVT z layer hit cut after the application of the lepton identification and track quality cuts.

\subsection{Selection of primary leptons}

After the application of all the above cuts, the two primary leptons were chosen. The two primary leptons were taken to be the two tracks with the highest $p^{*}$ that passed all the above cuts. 


\subsection{Selected event sample}

The selection efficiencies of the MC and data samples selected using the above cuts are presented in table 4.10. For signal MC, a selection efficiency of about $18 \%$ with a purity of $88 \%$ was obtained.

\begin{tabular}{|c|c|c|c|}
\hline Event Type & $\mathcal{L}\left(\mathrm{fb}^{-1}\right)$ & No of events selected & Selection $\mathcal{E}(\%)$ \\
\hline \hline Signal MC & 1.14 & 4370 & $18.20 \pm 0.30$ \\
$B^{0} \bar{B}^{0} \mathrm{MC}$ & 4.32 & 38621 & $1.70 \pm 0.01$ \\
$B^{+} B^{-} \mathrm{MC}$ & 4.52 & 37437 & $1.58 \pm 0.01$ \\
$c \bar{c} \mathrm{MC}$ & 2.68 & 3860 & $0.111 \pm 0.002$ \\
$u \bar{u}, s \bar{s}, d \bar{d} \mathrm{MC}$ & 1.72 & 504 & $0.014 \pm 0.001$ \\
\hline On-peak data & 7.72 & 142682 & $0.510 \pm 0.001$ \\
Off-peak data & 1.18 & 2490 & $0.083 \pm 0.002$ \\
\hline
\end{tabular}

Table 4.10: Selected events : Final selection efficiencies after the application of all the cuts. The selection efficiencies for the data sample was calculated using the events that passed the multihadron selection procedure outlined in section 4.4 .

\subsection{Chapter summary}

An introduction into the dilepton approached used by BABAR to calculate the mixing parameter, $\Delta m_{d}$ was given. The data sample used in this analysis consisted of $7.72 \mathrm{fb}^{-1}$ data collected by the BABAR detector from January to June 2000. An overview of the BABAR very tight electron and muon selectors was given. It was concluded that the electron and muon selectors had efficiencies of more than $80 \%$ and $60 \%$ respectively in the momentum region of interest to this analysis. The various sources of backgrounds affecting the measurement of $\Delta m_{d}$ and the cuts used to reduce the contamination from them were then given. The dominant backgrounds were shown to be due to cascade leptons arising from the semileptonic decay of the charm. With the cuts presented, it was shown that a selection efficiency of about $18 \%$ with a purity of $88 \%$ for signal MC was obtained. 


\section{Chapter 5}

\section{Extraction of $\Delta m_{d}$ and a study of the systematics}

\subsection{Introduction}

An overview of the dilepton analysis was given in chapter 4. In this section, the extraction of $\Delta m_{d}$ from the data will be presented. In doing this an unbinned maximum likelihood method will be used. It is expected that this approach would result in a smaller systematic error compared to the binned $\chi^{2}$ method used by BABAR for the ICHEP 2000 result [63].

The chapter will begin with an overview of the method employed to estimate the decay vertices of the two $\mathrm{B}$ mesons. This will be followed by a discussion of the expected vertex resolutions. The vertex resolutions will be calculated using two different methods; a global resolution function and an event-by-event error resolution function. The event-by-event error resolution function will be used in the measurement of $\Delta m_{d}$ and the results obtained will be crossed checked using the global resolution function. The parameterisations used to describe the backgrounds will be presented next. The extraction of $\Delta m_{d}$ from the data will be then presented. This section will contain a discussion of the likelihood fit used and the validation of the fit using MC. This will be followed by a discussion of the systematic effects affecting the measurement of $\Delta m_{d}$. The chapter will end with an overview of the stability of the fit and a short summary. 


\subsection{Estimation of the B decay vertex}

To estimate the decay vertices of the two B mesons, a point of closest approach (POCA) method was used. For each lepton track, the POCA of that track to the beamspot in the xy plane (figure 5.1) was calculated and was taken to be the position of the $\mathrm{B}$ decay vertex. The $\mathrm{z}$ position of the $\mathrm{B}$ decay vertex was thus taken to be the $\mathrm{z}$ coordinate of the POCA. This method, however, is sensitive to the beamspot parameters which are calculated on a run-by-run basis using Bhabha and di-muon events [64].

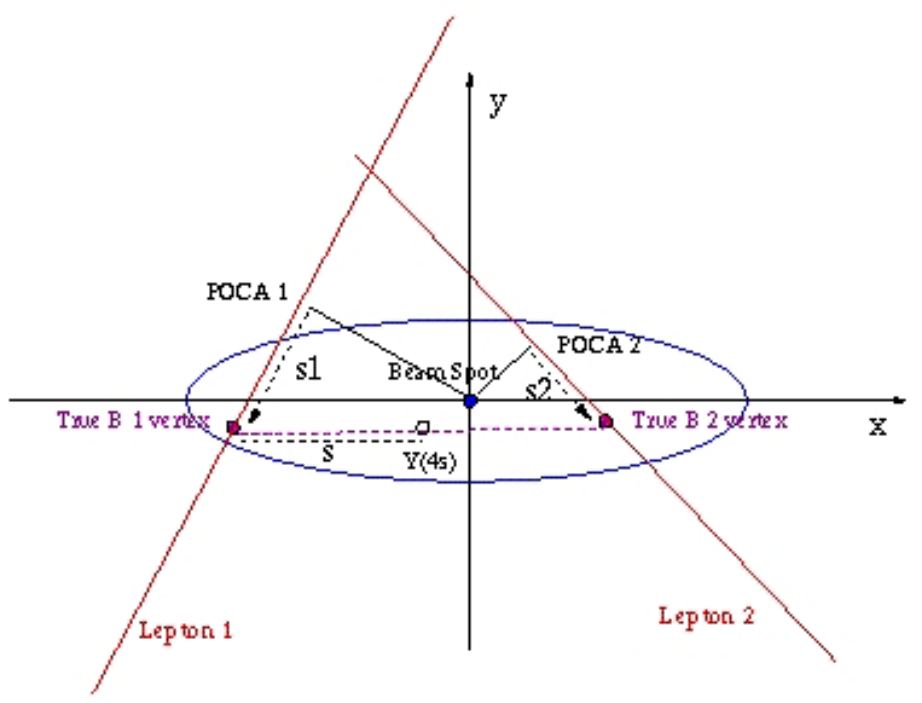

Figure 5.1: The estimation of the $B$ decay vertices using a POCA approach. The two $B$ decay vertices are taken to be the POCA of the two tracks to the nominal beam spot position.

\subsubsection{Calculation of $\Delta z$ and $\Delta t$}

Following the estimation of the two B decay vertices the difference between the two, $\Delta z$, was then calculated. As it is not possible to measure the time order of the two B decays, the definition of the sign of $\Delta z$ is arbitrary. For this analysis $\Delta z$ was defined to be,

$$
\Delta z=z_{\text {higher }}-z_{\text {other }}
$$


where $z_{\text {higher }}$ is the $\mathrm{z}$ coordinate of the higher $p^{*}$ primary lepton and $z_{\text {other }}$ is that of the other primary lepton. This method should not introduce any biases as the fit used in the extraction of $\Delta m_{d}$ is a function of $|\Delta z|$. The $\Delta z$ is related to the proper time, $\Delta t$ by the "boost approximation".

$$
\Delta t \approx \frac{\Delta z}{(<\gamma \beta>c)}
$$

In making this approximation, two assumptions are made. Firstly, it is assumed that the two $\mathrm{B}$ mesons are produced in the $\mathrm{z}$ direction and secondly that they are produced at rest in $\Upsilon(4 \mathrm{~S})$ rest frame. This gives a value of $\langle\gamma \beta\rangle=0.556$. The $\mathrm{B}$ meson are in fact not produced at rest but are done so with an momentum of about $340 \mathrm{MeV}$ in the $\Upsilon(4 \mathrm{~S})$ rest frame. The "boost approximation" is thus a source of systematic effects and this will be addressed in section 5.7.8. The absolute value of $\Delta t,|\Delta t|$, is then given by,

$$
|\Delta t| \approx \frac{|\Delta z|}{(<\gamma \beta>c)}
$$

\section{$5.3 \Delta \mathrm{z}$ resolution}

Before the physics functions describing the unmixed and mixed distributions can be fit to the data, they have to be convoluted with an appropriate resolution function. This resolution function has two main components. One is due to the resolution in the measurement of the z position in the SVT and the other is an intrinsic resolution due to the POCA method used in the estimation of the vertices. The residual in $\Delta z$ $\left(\Delta z_{\text {res }}\right)$ is defined to be,

$$
\Delta z_{\text {res }}=\Delta z_{\text {poca }}-\Delta z_{\text {true }}
$$

where $\Delta z_{\text {true }}$ is the true $\mathrm{B}$ decay separation and $\Delta z_{\text {poca }}$ is the $\mathrm{B}$ decay separation estimated using the POCA method outlined above. Two different approaches were use in the calculation of this resolution function. 


\subsubsection{Global resolution function}

In this method, the assumption was made that the $\Delta z$ resolution of the entire sample could be described by a single fixed resolution function. The resolution function was found to be best described by using three Gaussian distributions,

$$
\mathcal{G}_{\text {global }}\left(\Delta z_{\text {res }}\right)=\sum_{i=1}^{3} \frac{1}{\sqrt{2 \pi} \sigma_{i}} e^{\Delta z_{\text {res }}^{2} / 2 \sigma_{i}^{2}}
$$

The $\Delta z_{\text {res }}$ for true dileptons selected from generic $B^{0} \bar{B}^{0} \mathrm{MC}$ events can be seen in figure 5.2. The three Gaussian resolution function in equation 5.5 can be seen to describe the MC well. The sigmas of the narrow, wide and tail Gaussians were found to be, $110 \mu \mathrm{m}, 282 \mu \mathrm{m}$ and $639 \mu \mathrm{m}$ respectively. The fraction of events in the narrow Gaussian was found to be $43.3 \%$. The wide and tail Gaussians were found to contain $45.8 \%$ and $10.9 \%$ of the events respectively. The correlation ,matrix for the fit parameters is given in table 5.1.

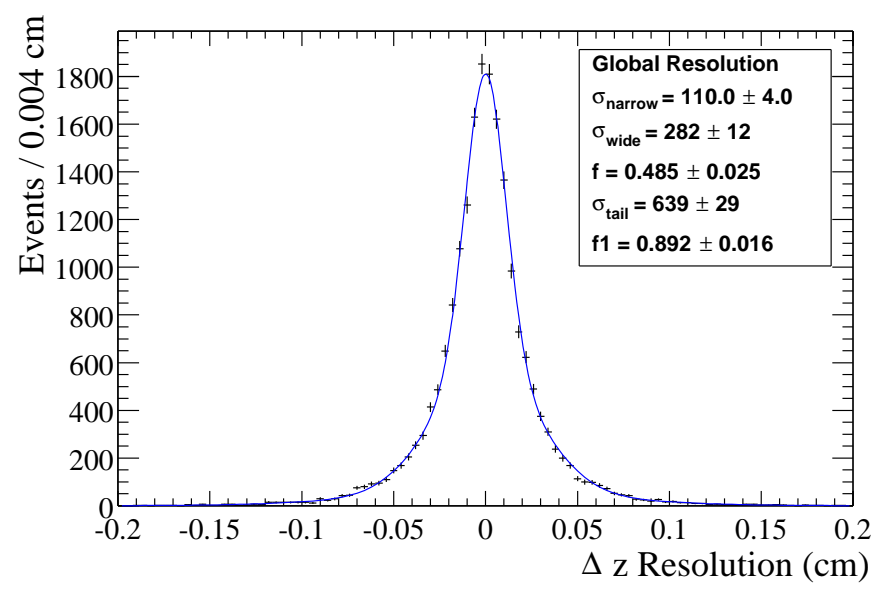

Figure 5.2: $M C$ Global $\Delta z$ resolution. The $M C$ data points were fit using three Gaussian distribution given in equation 5.5. The $\chi^{2} /$ d.o.f of the fit was found to be 1.43.

\subsubsection{Event error resolution function}

The second method used in the determination of the resolution function involved the use of the tracking errors. The $\Delta z_{\text {res }}$ given in equation 5.4 was weighted by the tracking errors and the event-by-event residual $\left(\Delta z_{\text {evt }}\right)$ was defined as, 


\begin{tabular}{|c|c|c|c|c|c|}
\hline & $\sigma_{\text {narrow }}$ & $\sigma_{\text {wide }}$ & $\mathrm{f}$ & $\sigma_{\text {tail }}$ & $\mathrm{f} 1$ \\
\hline \hline$\sigma_{\text {narrow }}$ & 1.000 & 0.799 & 0.898 & 0.476 & 0.564 \\
$\sigma_{\text {wide }}$ & 0.799 & 1.000 & 0.771 & 0.760 & 0.873 \\
$\mathrm{f}$ & 0.898 & 0.771 & 1.000 & 0.366 & 0.465 \\
$\sigma_{\text {tail }}$ & 0.476 & 0.760 & 0.366 & 1.000 & 0.911 \\
$\mathrm{f} 1$ & 0.564 & 0.873 & 0.465 & 0.911 & 1.000 \\
\hline
\end{tabular}

Table 5.1: Correlation matrix for the global resolution function fit parameters.

$$
\Delta z_{e v t}=\frac{\Delta z_{r e s}}{\sqrt{\left(\sigma_{t r k 1}^{2}+\sigma_{t r k 2}^{2}\right)}}
$$

where $\sigma_{t r k 1}$ and $\sigma_{t r k 2}$ are the tracking errors in $\mathrm{z}$ for the two primary leptons. The event error distribution was fit using a double Gaussian distribution given by,

$$
\mathcal{G}_{\text {event }}\left(\Delta z_{\text {evt }}\right)=\sum_{i=1}^{2} \frac{1}{\sqrt{2 \pi} \sigma_{i}} e^{\Delta z_{\text {evt }}^{2} / 2 \sigma_{i}^{2}}
$$

The result of the fit can be seen in figure 5.3. The distribution, once again, is for true dileptons selected from generic $B^{0} \bar{B}^{0} \mathrm{MC}$. The sigmas of the narrow and wide Gaussians were found to be $1.23 \pm 0.02$ and $2.57 \pm 0.06$ respectively, with about $72 \%$ of the events residing in the narrow Gaussian. The correlation matrix for the fit parameters is given in table 5.2 .

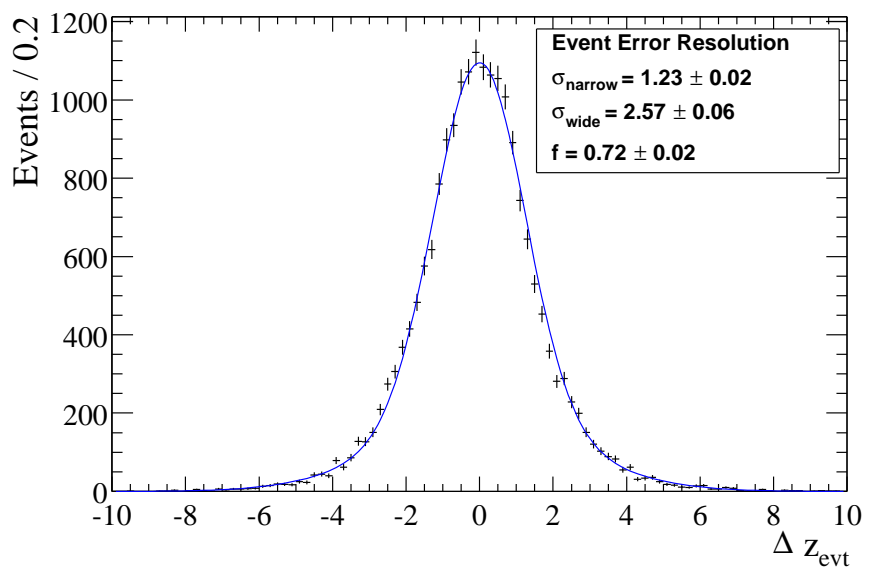

Figure 5.3: $M C$ event error resolution function. $M C$ data were fit using the double Gaussian distribution given in equation 5.7. The $\chi^{2} /$ d.o.f of the fit was found to be 1.49 . 


\begin{tabular}{|c|c|c|c|}
\hline & $\sigma_{\text {narrow }}$ & $\sigma_{\text {wide }}$ & $\mathrm{f}$ \\
\hline \hline$\sigma_{\text {narrow }}$ & 1.000 & 0.731 & 0.874 \\
$\sigma_{\text {wide }}$ & 0.731 & 1.000 & 0.882 \\
$\mathrm{f}$ & 0.874 & 0.882 & 1.000 \\
\hline
\end{tabular}

Table 5.2: Correlation matrix for the event error resolution function fit parameters.

A comparison between data and MC for the resolution function was made and the MC distribution was overlayed on the data. The resolution function for data was calculated using the decay vertices of $J / \psi \rightarrow e^{+} e^{-}$and $J / \psi \rightarrow \mu^{+} \mu^{-}$decays and the data chosen corresponded to a three sigma cut around the $J / \psi$ peak. Thus $z_{\text {true }}$ was taken to be the reconstructed decay vertex of the $J / \psi$. The resolution functions for the separate ee and $\mu \mu$ cases (figure 5.4) were found to be in agreement and thus a single resolution function was used in the analysis. The $\chi^{2} /$ d.o.f obtained by overlaying the MC resolution function given in figure 5.3 were found to be 1.12 and 1.19 for the ee and $\mu \mu$ cases respectively. The negative points in the tails of the distribution are due to background subtraction. The data used for this subtraction were required to lie in a three sigma mass region in the tails of the $J / \psi$ mass distribution.

\subsection{Discussion of the fit procedure}

\subsubsection{Method of maximum likelihood}

A maximum likelihood fitting procedure was used in this analysis. For a given probability density function (pdf), $F\left(x_{i} ; \vec{p}\right)$, that parameterises a set of $n$ data points, $x_{i}$, with a set of parameters, $\vec{p}$, the likelihood function $\mathcal{L}\left(x_{i} ; \vec{p}\right)$ is defined as,

$$
\mathcal{L}(\vec{p})=\prod_{i=1}^{n} F\left(x_{i} ; \vec{p}\right)
$$

The method of maximum likelihood involves finding the set of parameters that minimises the negative log likelihood function given by,

$$
-\log \mathcal{L}(\vec{p})=-\sum_{k} \log \left[F\left(x_{k} ; \vec{p}\right)\right]
$$



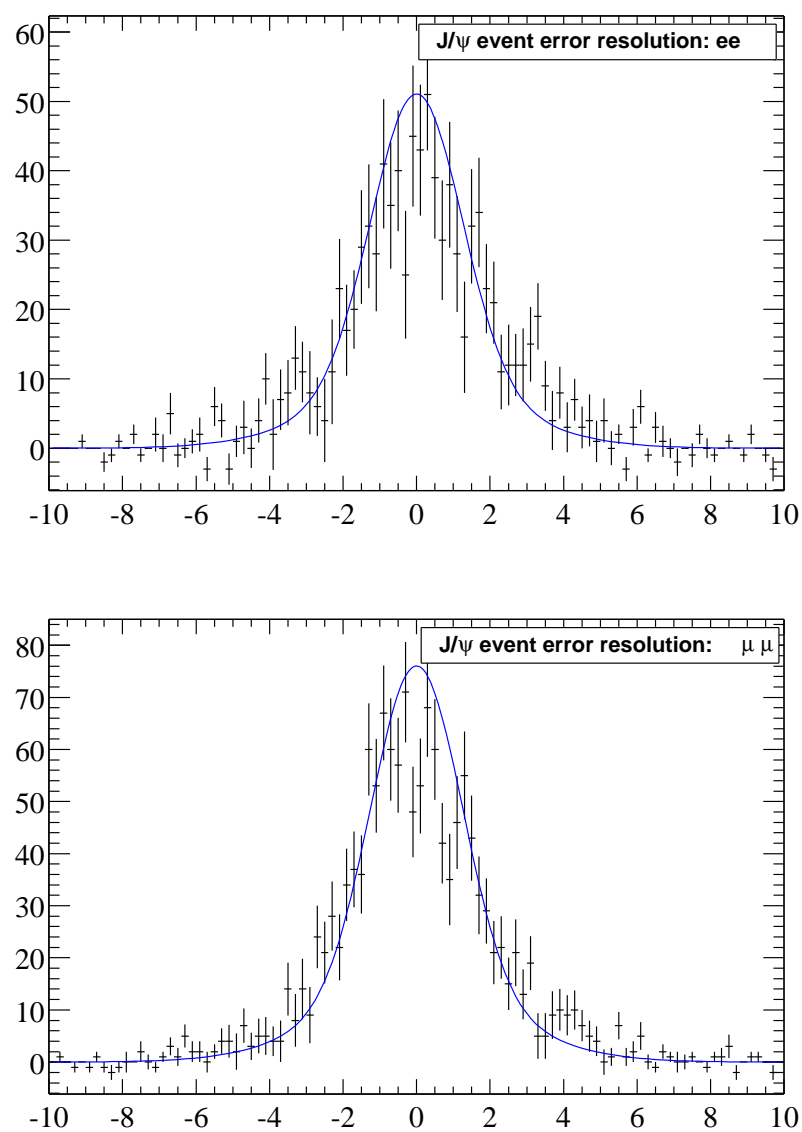

Figure 5.4: Overlaying of the MC resolution function given in figure 5.3 over the one calculated from data using $J / \psi$ decays to ee (top plot) and $\mu \mu$ (bottom plot) events. A $\chi^{2} /$ d.o.f 1.12 for the ee case and 1.19 for the $\mu \mu$ case was obtained.

When using the event-by-event error method with error scaling factors, $\epsilon_{i}$, the likelihood function becomes,

$$
\mathcal{L}(\vec{p})=\prod_{i=1}^{n} F\left(x_{i}, \epsilon_{i} ; \vec{p}\right)
$$

\subsubsection{Fit procedure for backgrounds}

It was found that most residual backgrounds in this analysis could be parameterised in terms of an exponential decay term which was convoluted with the detector resolution functions given in sections 5.3.1 and 5.3.2. A full discussion of the parameterisations of the different sources of backgrounds is presented in the following section. 
Before proceeding, the mathematical framework required for the parameterisation will be presented. Given an exponential decay pdf,

$$
\mathcal{P}(|\Delta t| ; \tau)=\frac{1}{\tau} e^{-|\Delta t| / \tau}
$$

and a resolution function,

$$
\mathcal{G}_{\text {res }}(\Delta t ; \sigma)=\frac{1}{\sqrt{2 \pi} \sigma} e^{-\Delta t^{2} / 2 \sigma^{2}}
$$

and per-event error scaling given by $\epsilon$, i.e. the total error is $\epsilon \sigma$, the convolution of the signal with the resolution function is defined as,

$$
\mathcal{P}_{f i t}\left(|\Delta t|^{\prime}, \epsilon ; \tau, \sigma\right)=N \int_{0}^{\infty} e^{-|\Delta t| / \tau} e^{-\left(|\Delta t|-|\Delta t|^{\prime}\right)^{2} / 2 \sigma^{2} \epsilon^{2}} d(|\Delta t|)
$$

The above can be solved analytically. Using the result derived in Appendix B, the convoluted pdf is given by,

$$
\mathcal{P}_{f i t}\left(|\Delta t|^{\prime}, \epsilon ; \tau, \sigma\right)=\frac{1}{2 \tau} e^{\left(\frac{\sigma^{2} \epsilon^{2}}{2 \tau^{2}}-\frac{|\Delta t|^{\prime}}{\tau}\right)} \operatorname{erfc}(\mathrm{y})
$$

where $\mathrm{y}$ is given by,

$$
y\left(|\Delta t|^{\prime}, \epsilon ; \tau, \sigma\right)=\frac{1}{\sqrt{2} \sigma \epsilon}\left(\frac{\sigma^{2} \epsilon^{2}}{\tau}-|\Delta t|^{\prime}\right)
$$

and the complementary error function $\operatorname{erfc}(\mathrm{y})$ is defined to be,

$$
\operatorname{erfc}(\mathrm{y})=\frac{2}{\sqrt{\pi}} \int_{\mathrm{y}}^{\infty} \mathrm{e}^{-\mathrm{u}^{2}} \mathrm{du}
$$

\subsection{Background characterisations}

A discussion of the parameterisation of the backgrounds used in the likelihood analysis will be given in this section. In doing this, the background contributions to the unmixed and mixed categories were handled separately. As stated in section 4.7.1, the cascade leptons from the semileptonic decays of the charm are the biggest source of background and this will be discussed first. 


\subsubsection{Cascade backgrounds}

The main contamination from cascades arise when one primary lepton is correctly identified and a cascade is incorrectly identified as the other primary. As previously stated (section 4.7.1), three forms of cascade backgrounds are present in this analysis with the dominant sources due to the $D^{ \pm}$and $D^{0} / \bar{D}^{0}$ mesons. Due to the difference in lifetime between the $D^{ \pm}\left(\tau_{D^{ \pm}}=1.051 \pm 0.013 \mathrm{ps}\right)$ and the $D^{0}\left(\tau_{D^{0}}=0.413 \pm\right.$ $0.003 \mathrm{ps}$ ) [26], and hence the semileptonic branching fractions, the contributions from the two were treated separately. The cascade parameterisation described below were calculated by using $B^{0} \bar{B}^{0} \mathrm{MC}$.

\section{$D^{ \pm}$backgrounds}

The cascade backgrounds from the charged D mesons were seen to provide the highest background contamination. The parameterisation of the simulated mixed $D^{ \pm}$cascade backgrounds is shown in figure 5.5. The background was parameterised using a single exponential term with $\tau=237 \pm 6 \mu \mathrm{m}$ convoluted with the event-byevent error resolution function given above.

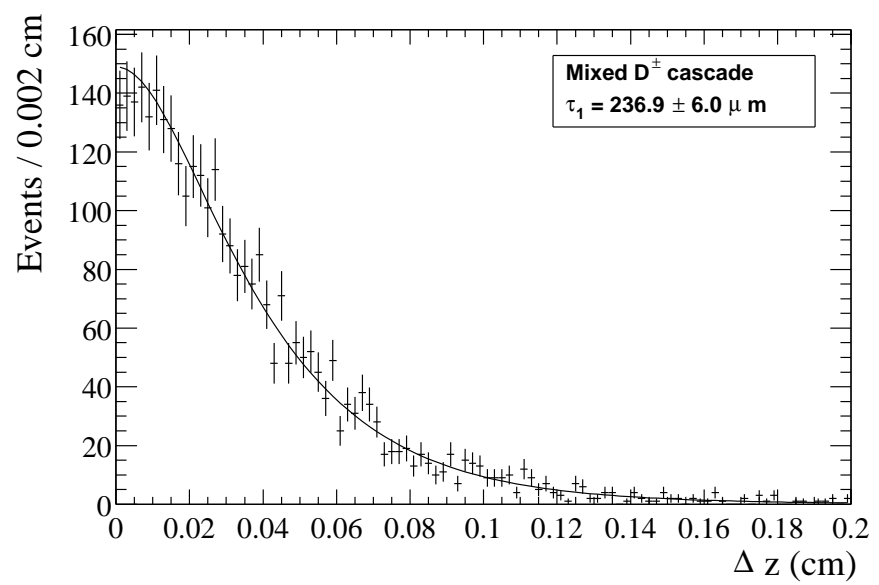

Figure 5.5: Mixed $M C D^{ \pm}$cascade background parameterisation. The fit was performed using the distribution given in equation 5.14. The $\chi^{2} /$ d.o.f of the fit was found to be 0.88 .

The parameterisation of the contribution to the unmixed category was complicated due to the presence of a non-negligible amount of contamination from cascades arising from the same B meson. As a result, two smeared exponential decay terms 
were used in the parameterisation. One with a very short lifetime to take into account the same B cascade contribution and the other, a longer lifetime term to describe the cascade from the other B. The result of the fit is shown in figure 5.6. The lifetimes of the two decay terms were found to be $130 \pm 19 \mu \mathrm{m}$ and $328 \pm 10 \mu \mathrm{m}$ with about $27 \%$ of the events in the distribution with the shorter lifetime. It should be noted that the short lifetime term of $130 \pm 19 \mu \mathrm{m}(0.78 \pm 0.11 \mathrm{ps})$ is within 2.5 sigma of the value of the charged D lifetime used in the simulation of $175 \mu \mathrm{m}$ (1.05 ps). In doing this conversion of the lifetime from units of $\mu \mathrm{m}$ to ps, the boost approximation was used and $\langle\gamma \beta\rangle$ was taken to be that of the $\mathrm{B}$ mesons. The total background contamination due to $D^{ \pm}$cascades was found to be $10.7 \%$.

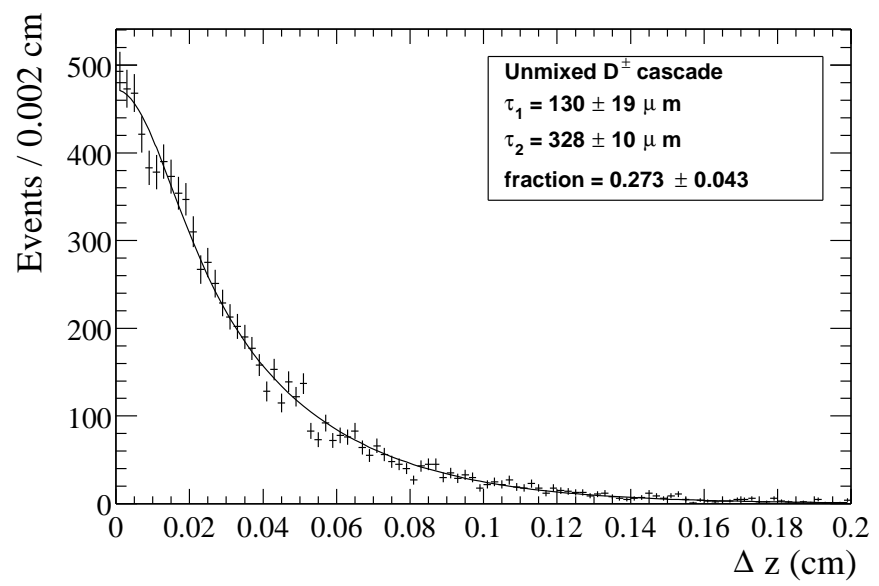

Figure 5.6: Unmixed $M C D^{ \pm}$cascade background parameterisation. The fit was performed using two exponential decay terms that were convoluted with the event-by-event resolution function. The $\chi^{2} /$ d.o.f of the fit was found to be 1.19 .

\section{$D^{0} / \bar{D}^{0}$ backgrounds}

The parameterisation of the contribution to the mixed category from the $D^{0} / \bar{D}^{0}$ cascade backgrounds is shown in figure 5.7. As in the $D^{ \pm}$case, the background was parameterised using an exponential term convoluted with the event-by-event error resolution function. The $\tau$ of the distribution was found to be $249 \pm 6 \mu \mathrm{m}$.

Once again, as was in the case for the charged D, two smeared exponential terms were used to describe the contribution to the unmixed category. The result of the fit is shown in figure 5.8 where the two lifetime terms were found to be $\tau_{1}=79 \pm 13 \mu \mathrm{m}$ 


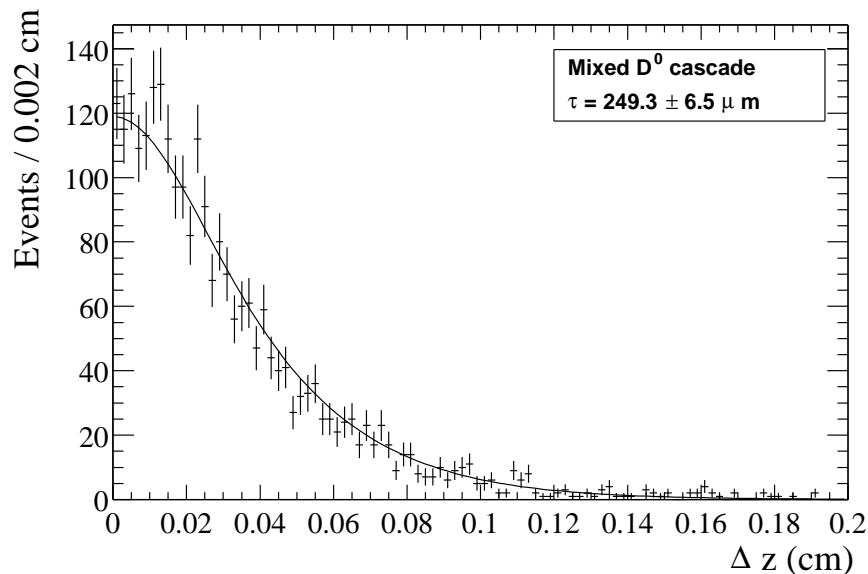

Figure 5.7: Mixed MC $D^{0}$ cascade background parameterisation. The expression given in equation 5.14 was fit to the data. The fit was seen to give a $\chi^{2} /$ d.o.f of 0.97 .

and $\tau_{2}=248 \pm 13 \mu \mathrm{m}$ with about $34 \%$ of the events falling in the distribution with the shorter lifetime. The short lifetime of $79 \pm 13 \mu \mathrm{m}$ which corresponds to $0.40 \pm 0.07 \mathrm{ps}^{-1}$ is in good agreement with the value of the $D^{0}$ lifetime used in the simulation of $68 \mu \mathrm{m}(0.407 \mathrm{ps})$. Once again, the boost approximation was used in doing this conversion from $\mu \mathrm{m}$ to $\mathrm{ps}$. The total contribution from $D^{0} / \bar{D}^{0}$ cascades was found to be $5.4 \%$.

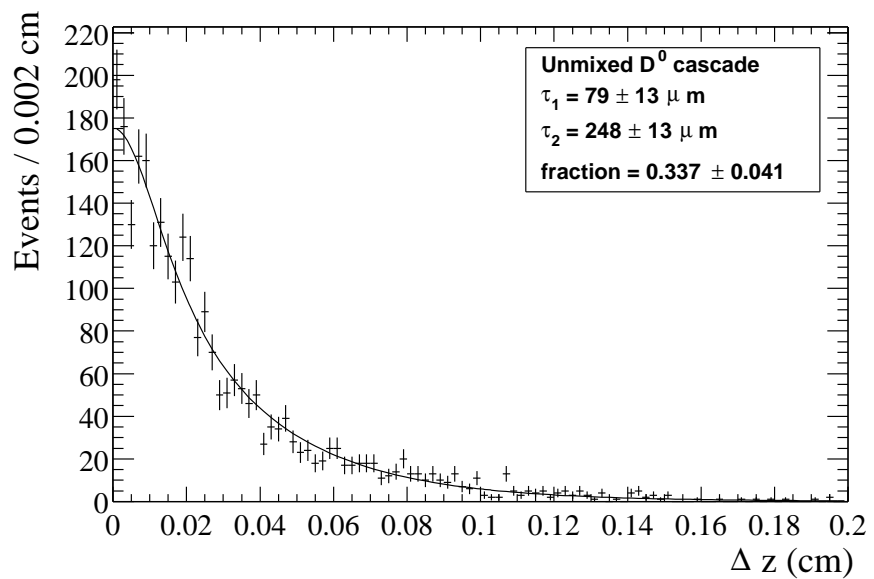

Figure 5.8: Unmixed $M C D^{0}$ cascade background parameterisation. The fit was performed using two distributions given by equation 5.14. The $\chi^{2}$ /d.o.f of the fit was found to be 1.06.

Any asymmetry resulting from the $\mathrm{B}$ meson in the primary-cascade mode was investigated. It was concluded that the asymmetry present was adequately described 
by the parameterisation derived above.

\subsection{2 $\quad B^{+} B^{-}$backgrounds}

Since the charged B mesons do not mix, dileptons arising from $B^{+} B^{-}$decays should only contribute to the unmixed category of events. Any contribution to the mixed category should arise from choosing a lepton from a charged B and another from cascades or other sources such as fake leptons. Both contributions were parameterised by using a smeared exponential term distributions as before. The results of the fit to the mixed case using a resolution smeared decay term with $\tau=245 \pm 4 \mu \mathrm{m}$ is shown in figure 5.9 .

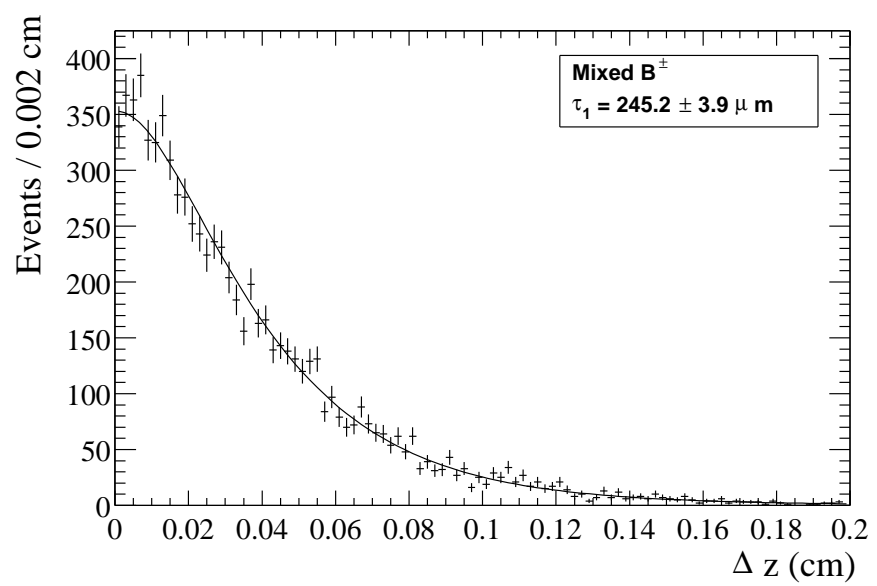

Figure 5.9: Mixed $M C B^{ \pm}$background parameterisation with a $\chi^{2} /$ d.o.f $=1.18$. The fit was performed using the distribution given in equation 5.14.

The contribution to the unmixed category was, once again, shown to be best described by using two smeared lifetime distributions to take into account the backgrounds from the cascade originating from the same B. The results of the fit are shown in figure 5.10. The lifetimes of the two terms were found to be $\tau_{1}=136 \pm 27 \mu \mathrm{m}$ and $\tau_{2}=260 \pm 4 \mu \mathrm{m}$ with about $11 \%$ of the events residing in the distribution with the shorter lifetime. The longer lifetime term of $260 \pm 4$ which corresponds to $1.56 \pm 0.03 \mathrm{ps}$ is within three $\sigma$ of the $B^{ \pm}$lifetime used in the simulation of $1.65 \mathrm{ps}$. The $B^{+} B^{-}$events were seen to constitute $46.6 \%$ of the full (generic $B^{0} \bar{B}^{0}+$ generic $B^{+} B^{-}+c \bar{c}+u \bar{u}+s \bar{s}+d \bar{d}$ )selected MC sample. 


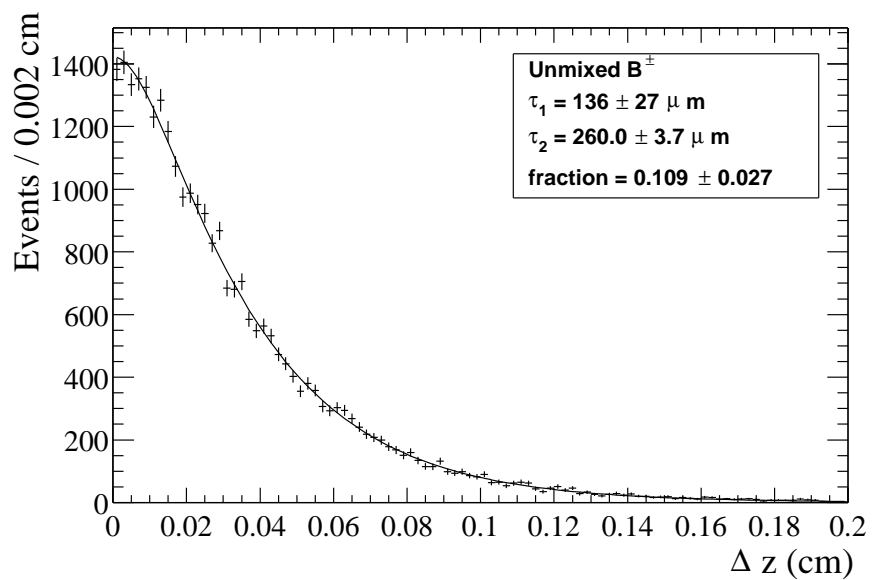

Figure 5.10: Unmixed $M C B^{ \pm}$background parameterisation. The data were fit to two convoluted exponential terms given in equation 5.14. The $\chi^{2} /$ d.o.f of the fit was found to be 1.13 .

\subsubsection{Continuum backgrounds}

The dominant contribution from continuum backgrounds was found to be from $c \bar{c}$ events. It was possible to parameterise the contribution to the mixed category by using a resolution smeared lifetime term as before. The result of the fit is shown in figure 5.12 .

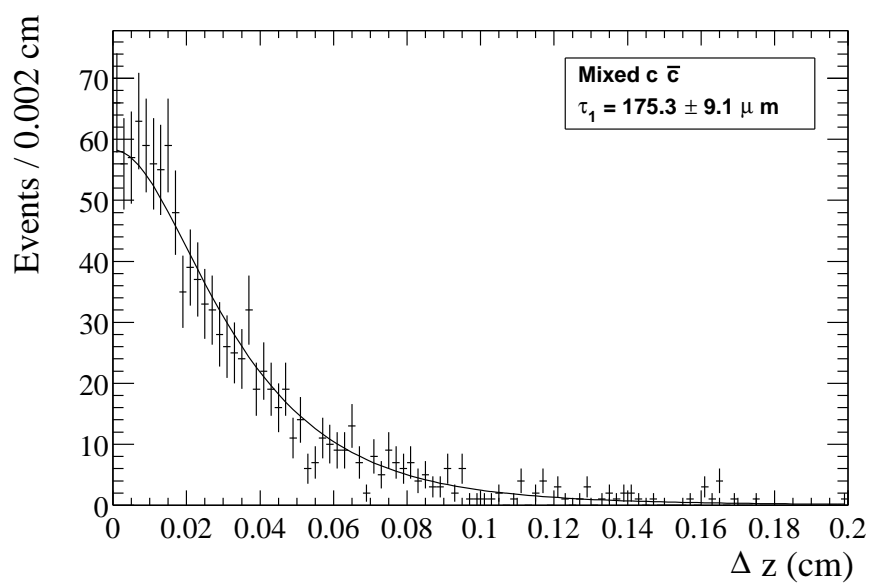

Figure 5.11: Mixed $M C c \bar{c}$ background parameterisation. The data were fit using an resolution smeared exponential decay term and the $\chi^{2} /$ d.o.f of the fit was found to be 0.80 .

The contribution to the unmixed case had to be described, once again, using two resolution smeared exponential terms, figure 5.11. About $17 \%$ of the events were found to lie in the shorter $\left(\tau_{1}=63 \pm 23 \mu \mathrm{m}\right)$ distribution and the rest in the longer 
lifetime $\left(\tau_{2}=190 \pm 11 \mu \mathrm{m}\right)$ distribution. The total fraction of $c \bar{c}$ events in the MC sample was found to be $4.8 \%$.

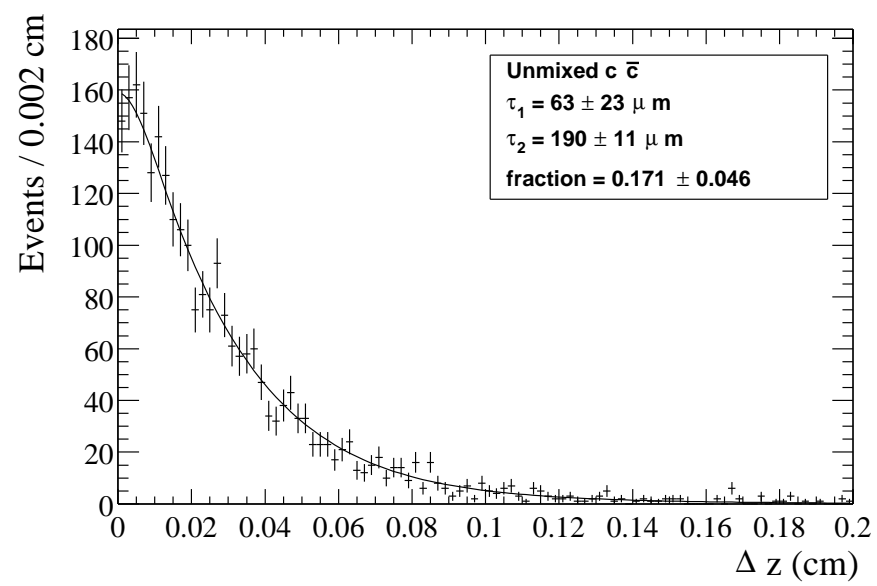

Figure 5.12: Unmixed $M C c \bar{c}$ background parameterisation. The $M C$ data were fit to two convoluted exponential terms. The $\chi^{2} /$ d.o.f of the fit is 0.90 .

A comparison between continuum MC and the off-resonance data is shown in figure 5.13. The mixed and unmixed parameterisations derived from MC were overlaid on the respective off-resonance distributions with no adjustment of the parameters. The $\chi^{2} /$ d.o.f obtained in doing this were found to be 0.75 and 0.80 respectively for the mixed and unmixed distributions. This process is a validation of the MC derived background parameterisations.

\subsubsection{Other backgrounds}

Other sources of backgrounds such as $u \bar{u}+d \bar{d}+s \bar{s}$ events $(0.6 \%)$, primary-fake $(0.9 \%)$, cascade-cascade $(0.5 \%)$, cascade-fake $(0.02 \%)$ and fake-fake $(0.01 \%)$ events were considered to be negligible and were not used in the fit.

\subsection{Measurement of $\Delta m_{d}$}

With the parameterisations of the backgrounds in place, it is possible to proceed with the extraction of $\Delta m_{d}$ from the data sample. A description of the likelihood fitting procedure used for this purpose will now be presented. The method involves a simultaneous unbinned maximum likelihood fit to the unmixed and mixed data samples. 

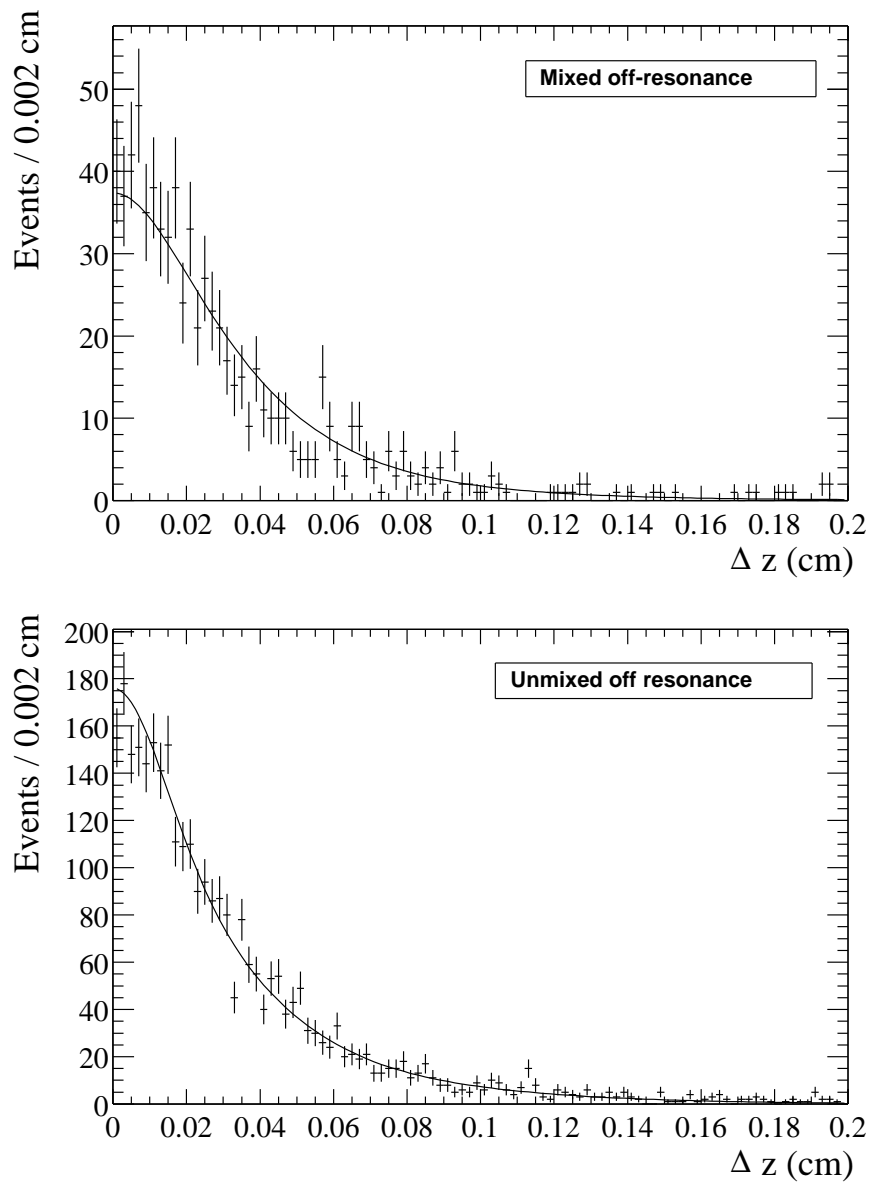

Figure 5.13: Overlaying of the MC continuum mixed (top figure) and unmixed (bottom figure) parameterisations over the off-resonance data with no adjustment of the parameters. The mixed distribution is overlaid with the exponential decay distribution given in figure 5.11 and the unmixed with the distribution given in figure 5.12. The $\chi^{2} /$ d.o.f of for the mixed and unmixed distributions were found to be 0.75 and 0.80 respectively.

\subsubsection{Probability density functions}

The pdfs describing the pure unmixed and mixed distributions are given by,

$$
\begin{aligned}
\mathcal{P}_{\text {unmixed }}(|\Delta t|) & \propto e^{-|\Delta t| / \tau_{B^{0}}}\left[1+D \cos \left(\Delta m_{d}|\Delta t|\right)\right] \\
\mathcal{P}_{\text {mixed }}(|\Delta t|) & \propto e^{-|\Delta t| / \tau_{B^{0}}}\left[1-D \cos \left(\Delta m_{d}|\Delta t|\right)\right]
\end{aligned}
$$

The dilution factor, D, can be expressed in terms of the mistag fraction, $\eta$, as

$$
D=1-2 \eta
$$


The expressions in equation 5.17 have to be convoluted with an appropriate resolution function described in sections 5.3 .1 and 5.3.2 before a fit to the data is made. The convolution integral is analytic and a full derivation can be found in Appendix C. The convolution of the unmixed and mixed pdfs with an event-by-event error double Gaussian resolution function with sigmas of $\sigma_{1}$ and $\sigma_{2}$ can be shown to be given by (Appendix C),

$$
\begin{array}{r}
\mathcal{P}_{\text {unmixed }}\left(|\Delta t|^{\prime}, \epsilon\right)=\mathcal{N}_{\text {unmixed }} \sum_{j=1}^{2}\left[e^{\left(\frac{\sigma_{j}^{2} \epsilon^{2}}{2 \tau^{2}}-\frac{|\Delta t|^{\prime}}{\tau}\right)} \operatorname{erfc}\left(\mathrm{y}_{\mathrm{j}}\right)\right. \\
\left.+\frac{D}{2} e^{-|\Delta t|^{\prime 2} / 2 \sigma_{j}^{2} \epsilon^{2}}\left(w\left(m_{j}\right)+w\left(-m_{j}^{*}\right)\right)\right]
\end{array}
$$

and

$$
\begin{array}{r}
\mathcal{P}_{\text {mixed }}\left(|\Delta t|^{\prime}, \epsilon\right)=\mathcal{N}_{\text {mixed }} \sum_{j=1}^{2}\left[e^{\left(\frac{\sigma_{j}^{2} \epsilon^{2}}{2 \tau^{2}}-\frac{|\Delta t|^{\prime}}{\tau}\right)} \operatorname{erfc}\left(\mathrm{y}_{\mathrm{j}}\right)\right. \\
\left.-\frac{D}{2} e^{-|\Delta t|^{\prime 2} / 2 \sigma_{j}^{2} \epsilon^{2}}\left(w\left(r_{j}\right)+w\left(-r_{j}^{*}\right)\right)\right]
\end{array}
$$

The per event error scaling factors are given by $\epsilon$ and $w(r)$ is the complex error function given by,

$$
w(r)=e^{-r^{2}} \operatorname{erfc}(-i r)
$$

$\operatorname{erfc}(r)$ is the complementary error function defined in equation 5.16. The functions $\mathrm{y}$ and $\mathrm{r}$ are given by,

$$
y_{j}\left(|\Delta t|^{\prime}, \epsilon\right)=\frac{1}{\sqrt{2} \sigma_{j} \epsilon}\left(\frac{\sigma_{j}^{2} \epsilon^{2}}{\tau}-|\Delta t|^{\prime}\right)
$$

and,

$$
r_{j}\left(|\Delta t|^{\prime}, \epsilon\right)=\frac{\sigma_{j} \epsilon \Delta m_{d}}{\sqrt{2}}+i\left(\frac{\sigma_{j} \epsilon}{\sqrt{2} \tau}-\frac{|\Delta t|^{\prime}}{\sqrt{2} \sigma_{j} \epsilon}\right)
$$

Finally, the normalisation factors, $\mathcal{N}_{\text {unmixed }}$ and $\mathcal{N}_{\text {mixed }}$ are given by, 


$$
\begin{aligned}
\mathcal{N}_{\text {unmixed }} & =\frac{1}{2}\left(\tau+\frac{D \tau}{1+\tau^{2} \Delta m_{d}^{2}}\right)^{-1} \\
\mathcal{N}_{\text {mixed }} & =\frac{1}{2}\left(\tau-\frac{D \tau}{1+\tau^{2} \Delta m_{d}^{2}}\right)^{-1}
\end{aligned}
$$

The background terms which were parameterised in the preceding section can then be added to the mixing pdfs with the appropriate normalisations. These normalisation factors describe the fraction of backgrounds in the sample. The normalisation factors for cascades, $c \bar{c}$ and $B^{+} B^{-}$were calculated from MC.

\subsubsection{Validation of the fit}

The fit function described in the previous section was validated using MC before the application to data. The MC sample used for this purpose was generated using the PDG 1996 value of $\Delta m_{d}$ of $0.464 \hbar \mathrm{ps}^{-1}$. The validation process was in three stages. The first stage involved fitting to the DOCA $|\Delta z|$ distribution of true primary leptons. This was done to validate the algorithms used in the convolution of the unmixed and mixed pdfs with the resolution function. The result of this can be seen in figure 5.14. The fit result of $0.467 \pm 0.007 \mathrm{~h} \mathrm{ps}^{-1}$ is in good agreement with the simulated value of $0.464 \mathrm{~h} \mathrm{ps}^{-1}$.

The second stage of this validation procedure involved the application of the fit with the appropriate cascade backgrounds to a sample of events from generic $B^{0} \bar{B}^{0}$ MC. These events were selected using the selection procedure described in chapter 4. This was done to validate the parameterisation and normalisations of the cascade backgrounds. The result of this fit is shown in figure 5.15. The fit result of $0.468 \pm$ $0.012 \mathrm{~h} \mathrm{ps}^{-1}$ is in very good agreement with the simulated value.

The final stage of the validation pipeline involved the application of the fit to a collection of generic $B^{0} \bar{B}^{0}, B^{+} B^{-}$and continuum MC events. This process is a validation of the parameterisations and normalisations of the $B^{ \pm}$and $c \bar{c}$ backgrounds. The result of this fit is shown in figure 5.16. The fit result of $0.472 \pm 0.017 \mathrm{~h} \mathrm{ps}^{-1}$ is in agreement with the value used in the simulation.

The parameterisations and background fractions derived from MC were used as constants in the above fits and the fit to the data. The MC validation fits thus 

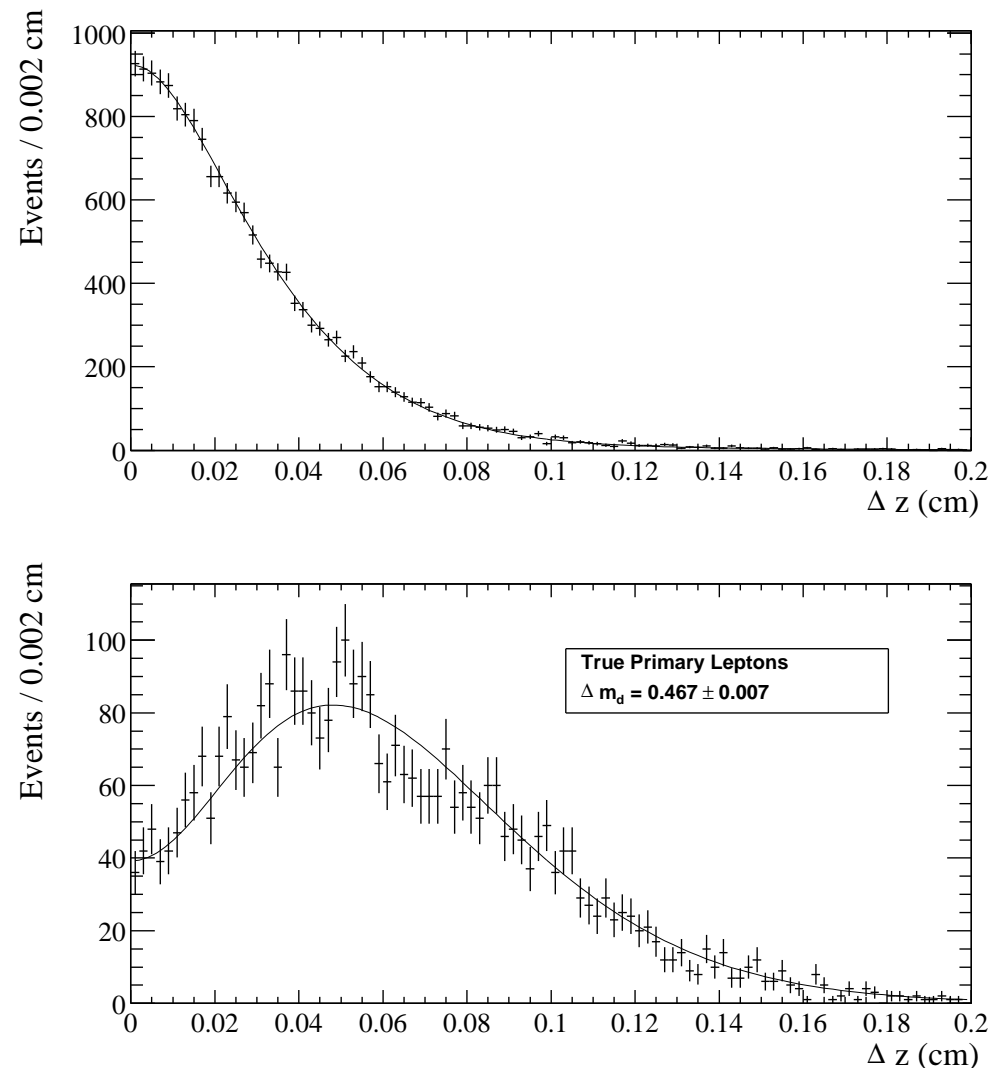

Figure 5.14: The $M C$ unmixed (top figure) and mixed (bottom figure) $|\Delta z|$ distributions for true primary leptons. The fit result of $0.467 \pm 0.007 \mathrm{~h} \mathrm{ps}{ }^{-1}$ is in good agreement with the simulated value of $0.464 \mathrm{~h} \mathrm{ps}^{-1}$. The $\chi^{2} /$ d.o.f of the fits were found to be, $\chi^{2} /$ d.o.f $f_{\text {unmixed }}=0.80$ and $\chi^{2} /$ d.o.f $f_{\text {mixed }}=1.08$.

consisted of one free parameter, $\Delta m_{d}$, whilst the final fit to the data consisted of two free parameters, $\Delta m_{d}$ and the mistag fraction, $\eta$.

\subsubsection{Data fit results}

The result of the fit to the data is shown in figure 5.17 and the resulting fit parameters are,

$$
\begin{aligned}
\Delta m_{d} & =0.466 \pm 0.018 \hbar \mathrm{ps}^{-1} \\
\eta & =0.211 \pm 0.008
\end{aligned}
$$

where the errors are purely statistical. The binned asymmetry distribution can be seen in figure 5.18. The asymmetry distribution was calculated using the fit 

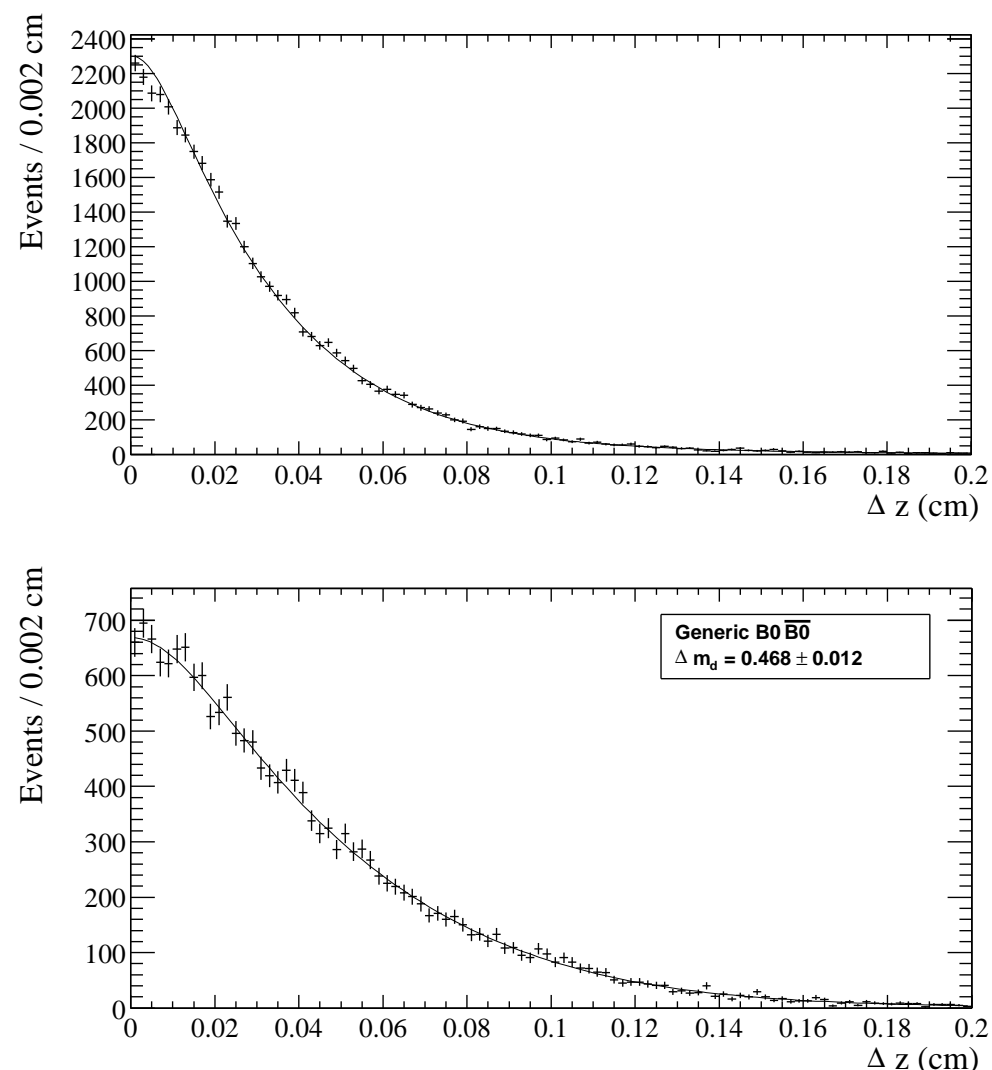

Figure 5.15: The $M C$ unmixed (top figure) and mixed (bottom figure) $|\Delta z|$ distributions for selected $B^{0} \bar{B}^{0}$ events. The fit result of $0.468 \pm 0.012 \mathrm{~h} p s^{-1}$ is in good agreement with the simulated value of $0.464 \mathrm{~h} \mathrm{ps}^{-1}$. The $\chi^{2} /$ d.o.f of the distributions were found to be 1.05 and 0.88 for the unmixed and mixed distributions respectively.

parameters given above.

\subsection{Systematic uncertainties}

The various sources of systematic uncertainties contributing to the analysis will now be presented. The main sources of systematic uncertainties will be described in the following sections and a summary of the full systematic effects can be found in table 5.8 .

\subsection{1 $\quad B^{0}$ lifetime}

The $B^{0}$ lifetime $\left(\tau_{B^{0}}\right)$ is a fixed parameter used in the fit (section 5.6) and the PDG 2000 value of $1.548 \pm 0.032$ ps was used. The systematic shift resulting in the 

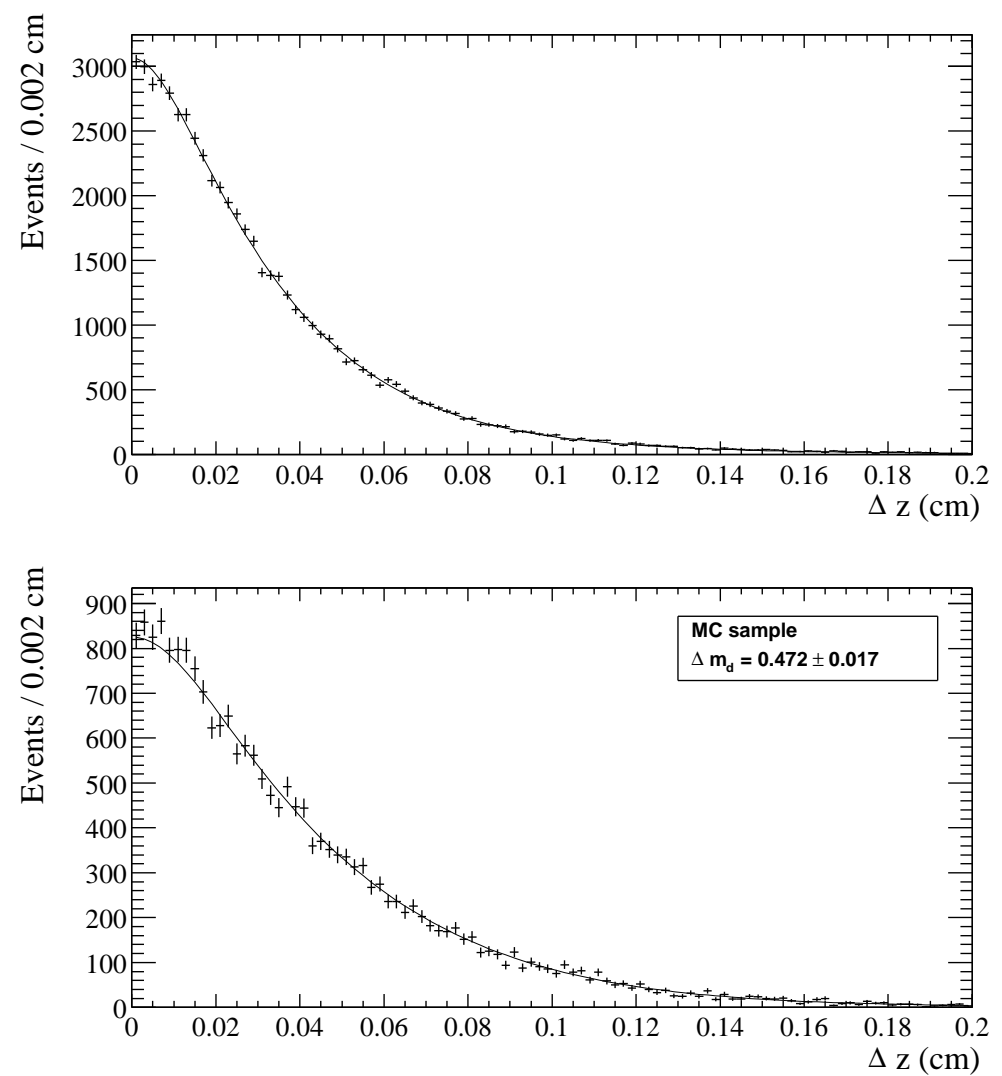

Figure 5.16: The $M C$ unmixed (top figure) and mixed (bottom figure) $|\Delta z|$ distributions for the full $M C$ sample including generic $B^{0} \bar{B}^{0}, B^{+} B^{-}$and continuum events. The result of the the fit of $0.472 \pm 0.017 \mathrm{~h} \mathrm{ps}^{-1}$ is in agreement with the simulated value of $0.464 \mathrm{~h} \mathrm{ps}^{-1}$. The $\chi^{2} /$ d.o.f of the unmixed and mixed distributions were found to be 0.97 and 1.37 respectively.

increase of $\tau_{B}$ by one sigma was found to be $0.008 \mathrm{~h} \mathrm{ps}^{-1}$.

\subsection{2 $\quad B^{ \pm}$lifetime}

The systematic shift resulting in the $B^{ \pm}$lifetime used in the simulation was investigated. In doing this, the $B^{ \pm}$background characterisation parameters (section 5.5.2) were each changed by $1.7 \%$ which corresponds to the PDG 2000 uncertainty in the $B^{ \pm}$lifetime. This corresponded to a shift in $\Delta m_{d}$ of $0.009 \mathrm{~h} \mathrm{ps}^{-1}$.

\subsubsection{Resolution function parameters}

The event-by-event error resolution function was defined in section 5.3.2. The parameters of this function are thus a source of systematic uncertainty. A summary of the systematic effects due to this resolution function is given in table 5.3. Due to 

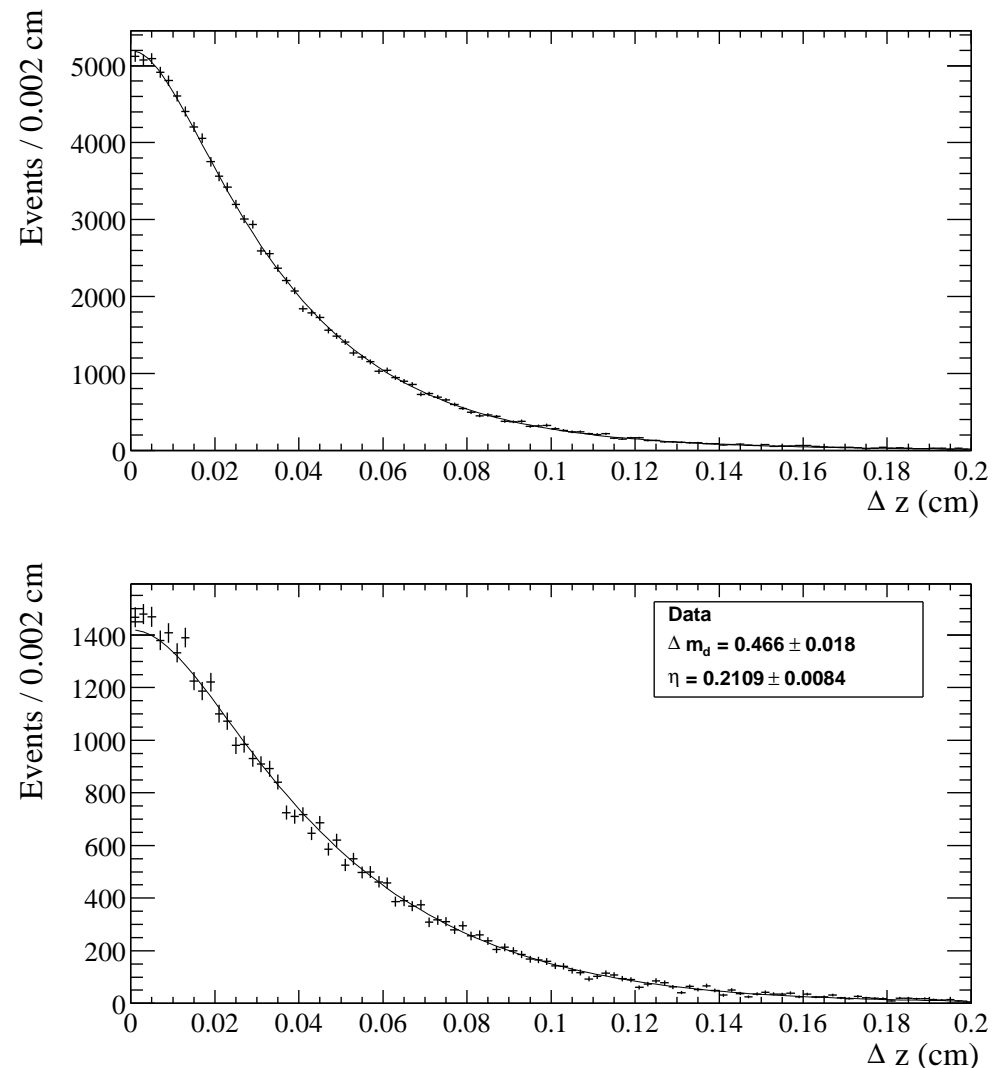

Figure 5.17: Unmixed (top figure) and mixed (bottom figure) $|\Delta z|$ distributions for the data. $A$ value of $0.466 \pm 0.018 \mathrm{\hbar} \mathrm{ps}^{-1}$ for $\Delta m_{d}$ and a value of $0.211 \pm 0.008$ for the mistag was obtained. The $\chi^{2} /$ d.o.f of the unmixed and mixed distributions were found to be 1.05 and 1.43 respectively.

the high correlation between the parameters, the total systematic error is calculated using the full error matrix analysis.

\section{Central Gaussian $\sigma$}

As seen in figure 5.3, the $\sigma$ of the narrow Gaussian $\left(\sigma_{\text {narrow }}\right)$ in the resolution function was found to be $1.23 \pm 0.02$. The systematic shift due to a change in $\sigma_{\text {narrow }}$ of 0.02 was found to be $0.007 \mathrm{~h} \mathrm{ps}^{-1}$.

\section{Wide Gaussian $\sigma$}

The systematic shift induced by changing the value of $\sigma_{\text {wide }}(2.57 \pm 0.06)$ in the resolution function by one $\sigma$ was calculated to be $0.007 \mathrm{~h} \mathrm{ps}{ }^{-1}$. 


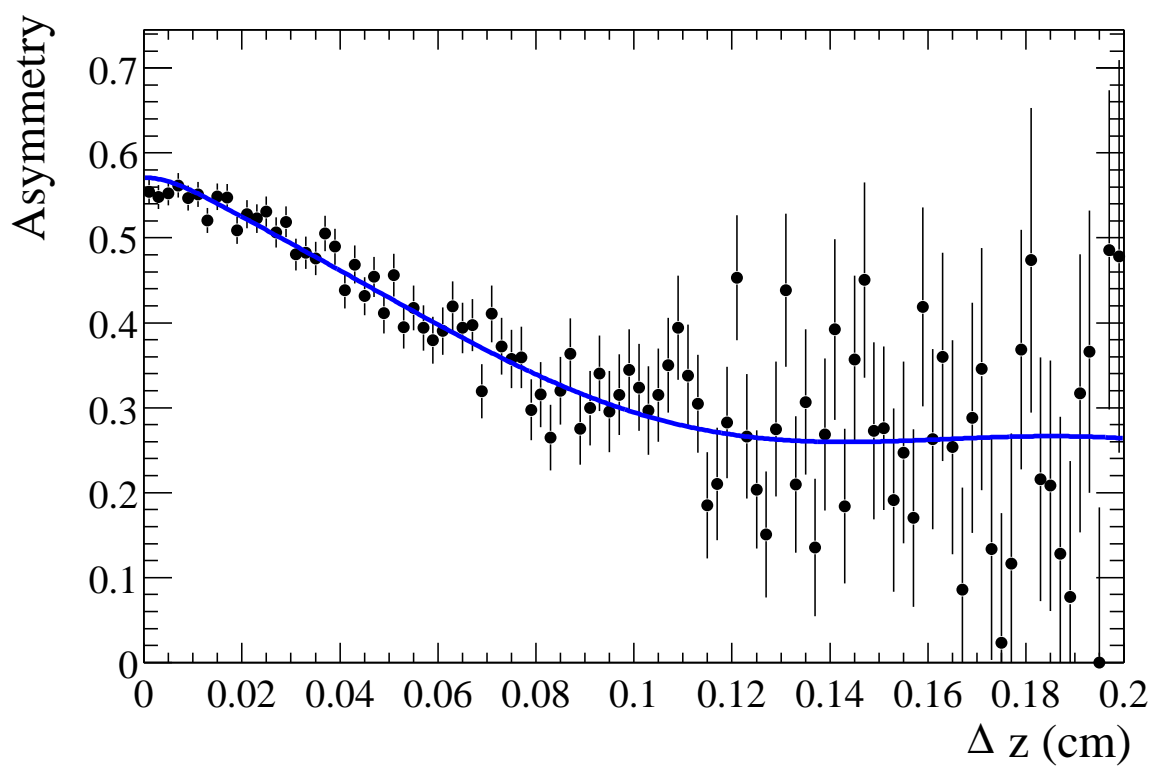

Figure 5.18: Asymmetry distribution for the data. The asymmetry curve is calculated using the fit parameters obtained from the simultaneous maximum likelihood fit to the unmixed and mixed distributions.

\section{Fraction of events in the central Gaussian}

The fraction of events in the narrow Gaussian was found to be 0.74. Changing this by one $\sigma(0.02)$, a shift in $\Delta m_{d}$ of $0.009 \mathrm{~h} \mathrm{ps}^{-1}$ was obtained.

\begin{tabular}{|c|c|}
\hline Systematic Error Type & $\sigma\left(\Delta m_{d}\right) \bar{h} \mathrm{ps}^{-1}$ \\
\hline \hline$\sigma_{\text {narrow }}$ & 0.007 \\
$\sigma_{\text {wide }}$ & 0.007 \\
Fraction of events in the narrow & 0.009 \\
\hline Total & 0.005 \\
\hline
\end{tabular}

Table 5.3: Systematic errors for the resolution function parameters. The errors are for a one $\sigma$ shift in each parameter. Due to the correlation between the parameters, the errors are not added in quadrature. The total is thus calculated using the full error matrix.

\subsubsection{Global resolution function}

Using the global resolution function defined in section 5.3.1, $\Delta m_{d}$ was measured to be $0.464 \pm 0.020 \mathrm{~h} \mathrm{ps}^{-1}$. 


\subsubsection{Lepton mis-identification}

The systematic uncertainty arising due to lepton mis-identification was calculated using MC. Here, the error was taken to be the difference in the result obtained using the full MC fit (figure 5.16) and that using true leptons with no constraints on the parent type of the lepton. A shift in $\Delta m_{d}$ of $0.008 \mathrm{~h} \mathrm{ps}^{-1}$ was observed.

\subsubsection{Backgrounds characterisation systematics}

The backgrounds from secondaries, $B^{+} B^{-}$and $c \bar{c}$ events were characterised by using lifetime distributions. The parameters of the fits are thus a source of systematic uncertainty and the uncertainty arising from them will now be addressed.

\section{Cascade background characterisation}

The systematics arising from the characterisation of the cascade background sources are summarised in table 5.4. The results are for a shift of one $\sigma$ in the fit parameters. The total resulting systematic shift due to cascade parameterisation was calculated to be $0.006 \mathrm{hps}^{-1}$

\begin{tabular}{|c|c|}
\hline Systematic Error Type & $\sigma\left(\Delta m_{d}\right) \overline{\mathrm{p}} \mathrm{ps}^{-1}$ \\
\hline \hline$D^{ \pm}$unmixed $\tau_{1}$ & 0.004 \\
$D^{ \pm}$unmixed $\tau_{2}$ & $\ll 0.001$ \\
$D^{ \pm}$unmixed fraction & $\ll 0.001$ \\
$D^{0} / \bar{D}^{0}$ unmixed $\tau_{1}$ & 0.001 \\
$D^{0} / \bar{D}^{0}$ unmixed $\tau_{2}$ & 0.001 \\
$D^{0} / \bar{D}^{0}$ unmixed fraction & 0.003 \\
$D^{ \pm}$mixed $\tau$ & 0.001 \\
$D^{0} / \bar{D}^{0}$ mixed $\tau$ & 0.002 \\
\hline Total & 0.006 \\
\hline
\end{tabular}

Table 5.4: Systematic errors for cascade background characterisations. The errors are for s shift of one $\sigma$ in the fit parameters. The parameters $\tau_{1}$ and $\tau_{2}$ correspond to the short and long lifetime terms used in the parameterisation of the unmixed backgrounds.

\section{$B^{+} B^{-}$and $c \bar{c}$ background characterisation}

The contribution to the systematic uncertainty from the characterisation of the $B^{+} B^{-}$and $c \bar{c}$ backgrounds is summarised in tables 5.5 and 5.6 respectively. Once 
again, the errors are calculated for a shift of one $\sigma$ in the fit parameters. The systematic shift from $B^{+} B^{-}$and $c \bar{c}$ parameterisations were found to be $0.005 \hbar \mathrm{ps}^{-1}$ and $0.003 \mathrm{~h} \mathrm{ps}^{-1}$ respectively.

\begin{tabular}{|c|c|}
\hline Systematic Error Type & $\sigma\left(\Delta m_{d}\right) \hbar \mathrm{ps}^{-1}$ \\
\hline \hline$B^{+} B^{-}$unmixed $\tau_{1}$ & 0.002 \\
$B^{+} B^{-}$unmixed $\tau_{2}$ & 0.004 \\
$B^{+} B^{-}$unmixed fraction & 0.001 \\
$B^{+} B^{-}$mixed $\tau$ & 0.002 \\
\hline Total & 0.005 \\
\hline
\end{tabular}

Table 5.5: Systematic errors for background characterisations of $B^{+} B^{-}$. The errors are for a shift of one $\sigma$ in the fit parameters.

\begin{tabular}{|c|c|}
\hline Systematic Error Type & $\sigma\left(\Delta m_{d}\right) \overline{\mathrm{ps}}{ }^{-1}$ \\
\hline \hline$c \bar{c}$ unmixed $\tau_{1}$ & 0.002 \\
$c \bar{c}$ unmixed $\tau_{2}$ & 0.001 \\
$c \bar{c}$ unmixed fraction & $\ll 0.001$ \\
$c \bar{c}$ mixed $\tau$ & 0.002 \\
\hline Total & 0.003 \\
\hline
\end{tabular}

Table 5.6: Systematic errors for background characterisations of $c \bar{c}$. The errors are for a shift of one $\sigma$ in the fit parameter.

\subsubsection{Background fractions}

The systematic effects resulting from changing the background fractions in the fit are summarised in table 5.7. For $B^{+} B^{-}$events, the results are for a shift of one $\sigma$ in the fractions. The $\sigma$ was calculated by running the full data fit with the $B^{ \pm}$ fractions as free parameters. For the cascades, the results are for shifts of $5 \%$ and $2.5 \%$ for $D^{ \pm}$and $D^{0} / \bar{D}^{0}$ respectively. These numbers correspond to one half of the background fractions used. For the continuum $c \bar{c}$ the shift corresponds to a $2 \%$ change in the fractions. The total systematic shift due to background fractions was found to be $0.007 \mathrm{~h} \mathrm{ps}^{-1}$.

\subsubsection{Boost approximation}

As shown in section 5.2, the proper time between the two B decays was calculated from the the $\Delta z$ using the boost approximation. From MC, the systematic shift due 


\begin{tabular}{|c|c|}
\hline Systematic Error Type & $\sigma\left(\Delta m_{d}\right) \overline{\mathrm{h}} \mathrm{ps}^{-1}$ \\
\hline \hline Fraction of $D^{ \pm}$unmixed $(5 \%)$ & 0.002 \\
Fraction of $D^{ \pm}$mixed $(5 \%)$ & $\ll 0.001$ \\
Fraction of $D^{0} / \bar{D}^{0}$ unmixed $(2.5 \%)$ & 0.003 \\
Fraction of $D^{0} / \bar{D}^{0}$ mixed $(2.5 \%)$ & $\ll 0.001$ \\
Fraction of $c \bar{c}$ unmixed $(2 \%)$ & 0.003 \\
Fraction of $c \bar{c}$ mixed $(2 \%)$ & 0.002 \\
Fraction of $B^{+} B^{-}$unmixed $(1 \sigma)$ & 0.004 \\
Fraction of $B^{+} B^{-}$mixed $(1 \sigma)$ & 0.001 \\
\hline Total & 0.007 \\
\hline
\end{tabular}

Table 5.7: Systematic errors due to background fractions. The errors are for a shift of one $\sigma$ in the fit parameters

to this approximation was calculated to be $0.001 \mathrm{~h} \mathrm{ps}^{-1}$ [63].

\subsubsection{Beamspot parameters}

When estimating the B decay vertex, the POCA of the tracks are calculated relative to the beamspot. Thus, the beamspot parameters are a potential source of systematic errors. The contribution to the systematic error from the beamspot parameters was found to be $\ll 0.001$.

\subsubsection{Simulation deficiencies}

The resulting systematic effects from simulation deficiencies were not considered in this analysis.

\subsubsection{Summary of systematic errors}

A full summary of the systematic errors can be found in table 5.8. The total systematic error for this analysis was found to be $0.019 \mathrm{~h} \mathrm{ps}^{-1}$.

\subsection{Consistency checks}

The stability of the fit with respect to the lepton sub-samples and data sub-samples was investigated. The results of the fit for ee, $\mu \mu$, e $\mu$ and $\mu e$ sub-samples are shown in figure 5.19. The first lepton refers to the dilepton with the higher $p^{*}$. Thus, e $\mu$ 


\begin{tabular}{|c|c|}
\hline Systematic Error Type & $\sigma\left(\Delta m_{d}\right) \hbar \mathrm{ps}^{-1}$ \\
\hline \hline Sensitivity to $\tau_{B^{0}}$ & 0.008 \\
Sensitivity to $\tau_{B^{ \pm}}$ & 0.009 \\
Resolution function & 0.005 \\
Lepton misidentification & 0.008 \\
Total background characterisation (cascade) & 0.006 \\
Total background characterisation $\left(B^{+} B^{-}\right)$ & 0.005 \\
Total background characterisation $(c \bar{c})$ & 0.003 \\
Total background fractions & 0.007 \\
Boost approximation & 0.001 \\
Beamspot parameters & $\ll 0.001$ \\
\hline Total & 0.019 \\
\hline
\end{tabular}

Table 5.8: Systematic error summary

refers to the case where the $p^{*}$ of the electron was higher than that of the muon. The results can be seen to be in agreement with the full fit result.

The fit results for the different data sub-samples are shown in figure 5.20. Here, the second data sample ("Osaka 2") which corresponds to $0.53 \mathrm{fb}^{-1}$ of data, yields a result that is within two sigma of the fit result.

The fit results as a function of the lower bound on the $p^{*}$ cut is shown in figure 5.21. The results were derived using the background characterisations and fractions calculated from MC for the different $p^{*}$ ranges. As can be seen, a low $p^{*}$ cut of about $0.9 \mathrm{GeV} / \mathrm{c}$ is outside the stability region of $\Delta m_{d}$. The stability region is defined as the region over which $\Delta m_{d}$ has no dependence on $p^{*}$. The cut of $1.2 \mathrm{GeV} / \mathrm{c}$ used in this analysis is well within the stability region and this was the main motivation for using such a high $p^{*}$ cut which resulted in a $38 \%$ loss in the signal efficiency (section 4.7.1).

A comparison between this measurement and previous measurement is shown in figure 5.22. The average given is the ICHEP 2000 value calculated using the LEP, SLD and CDF results and it does not include the BABAR or Belle measurements. The BABAR dilepton measurement was made using a binned $\chi^{2}$ fit to the asymmetry distribution. A summary of the Belle measurement made using a binned maximum likelihood method with a lower bound on the $p^{*}$ cut of $1.1 \mathrm{GeV} / \mathrm{c}$ can be found at [65]. 


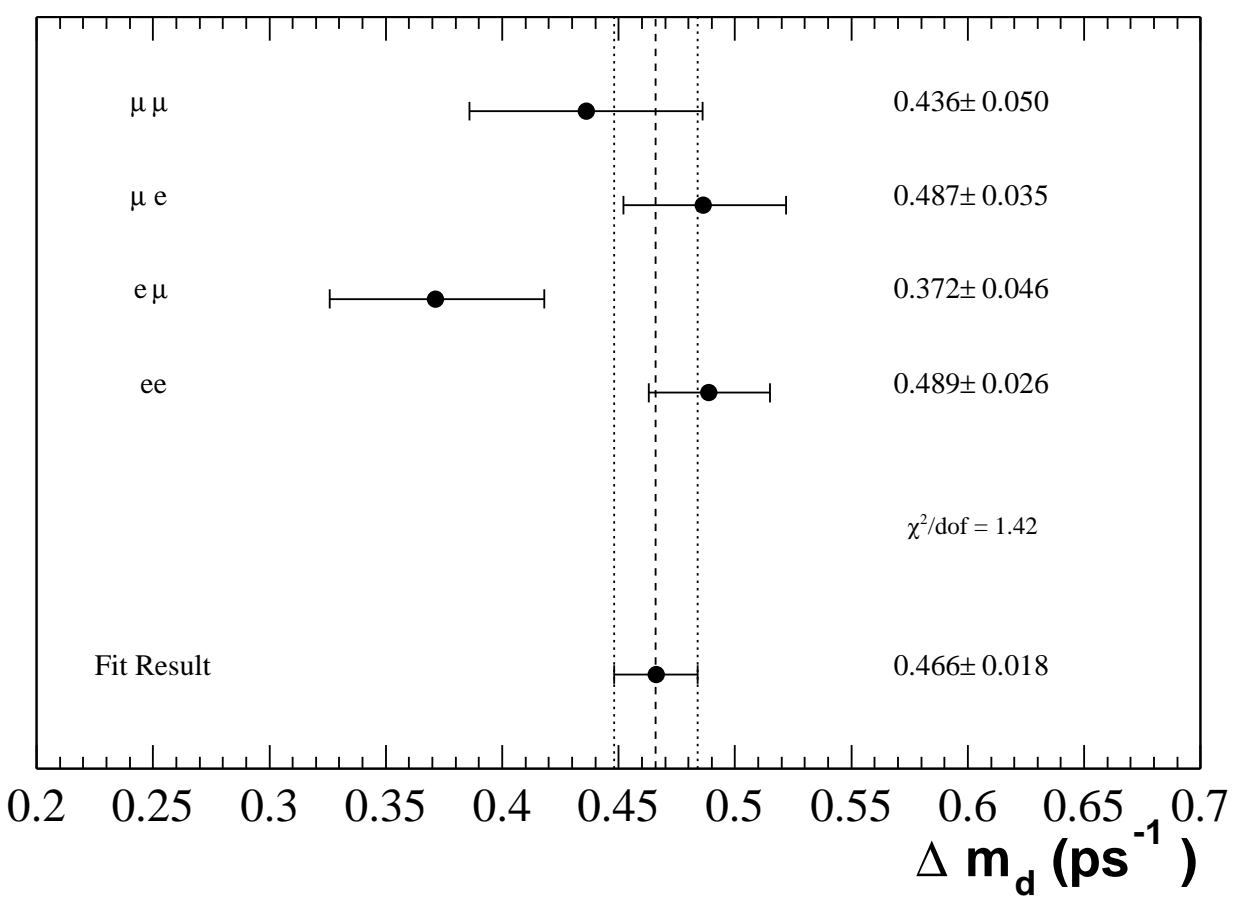

Figure 5.19: $\Delta m_{d}$ results for the ee, e $\mu, \mu e, \mu \mu$ lepton sub-samples. The first letter corresponds to the higher $p^{*}$ lepton. Thus, e $\mu$ refers to the case where the $p^{*}$ of the electron was higher than that of the muon.

\subsection{Chapter summary}

An overview of the POCA approach used to estimate the B decay vertices in the dilepton analysis was given. The event-by-event error resolution function that was used in the analysis was defined and calculated from MC. It was shown that the proper time between the two $\mathrm{B}$ decays was related to the difference in the $\mathrm{z}$ coordinates of the two vertices by a very simple relationship.

An introduction into the method of maximum likelihood was given. The convolution of the lifetime terms used in the parameterisations with the response function was shown to be analytic. The parameterisations used to describe the residual backgrounds in the analysis were presented. It was concluded that the contribution to the mixed category from cascade, $B^{+} B^{-}$and $c \bar{c}$ events could be described by an exponential term that was smeared by the resolution function. The contribution to the unmixed case was, however, found to be further complicated due to the presence of a non-negligible contamination from the cascade originating from the same B. These 


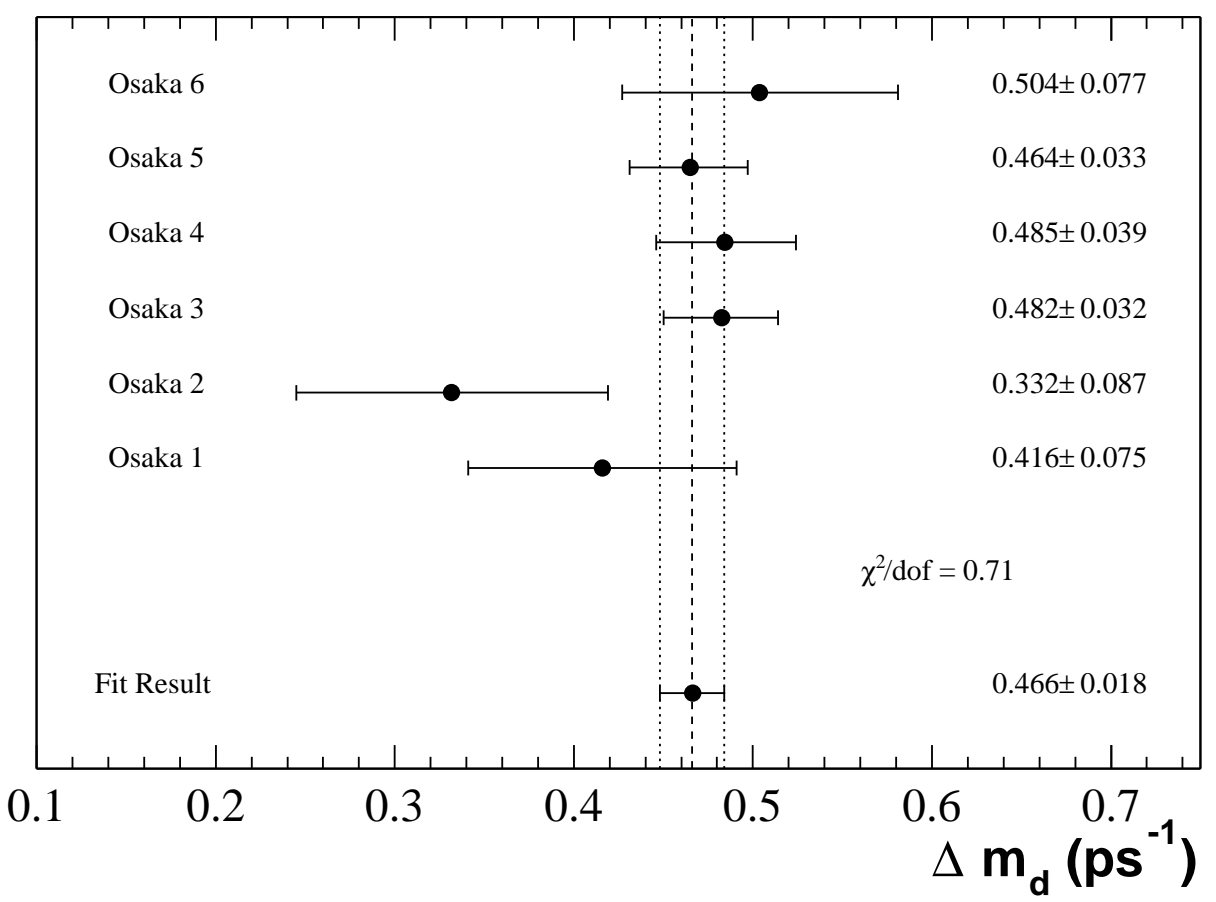

Figure 5.20: $\Delta m_{d}$ results for the different data sub-samples ("Osaka samples") used in the analysis. A summary of the luminosities corresponding to each sub-sample is given in table 4.2.

distributions were therefore parameterised using two smeared lifetime terms.

The analytical forms of the convoluted unmixed and mixed distributions were then given. Following the validation of the fit using MC events, the fit results for data were presented. The mixing parameter, $\Delta m_{d}$, was found to be $0.466 \pm 0.018 \mathrm{~h} \mathrm{ps}^{-1}$. The mistag fraction was found to be $0.211 \pm 0.008$. A discussion on the various sources of systematic uncertainties was then presented. The total systematic error was calculated to be $0.019 \mathrm{~h} \mathrm{ps}^{-1}$. The full result of $\Delta m_{d}$ including the systematic errors was then found to be

$$
\Delta m_{d}=0.466 \pm 0.018 \pm 0.019 \mathrm{hps}^{-1}
$$




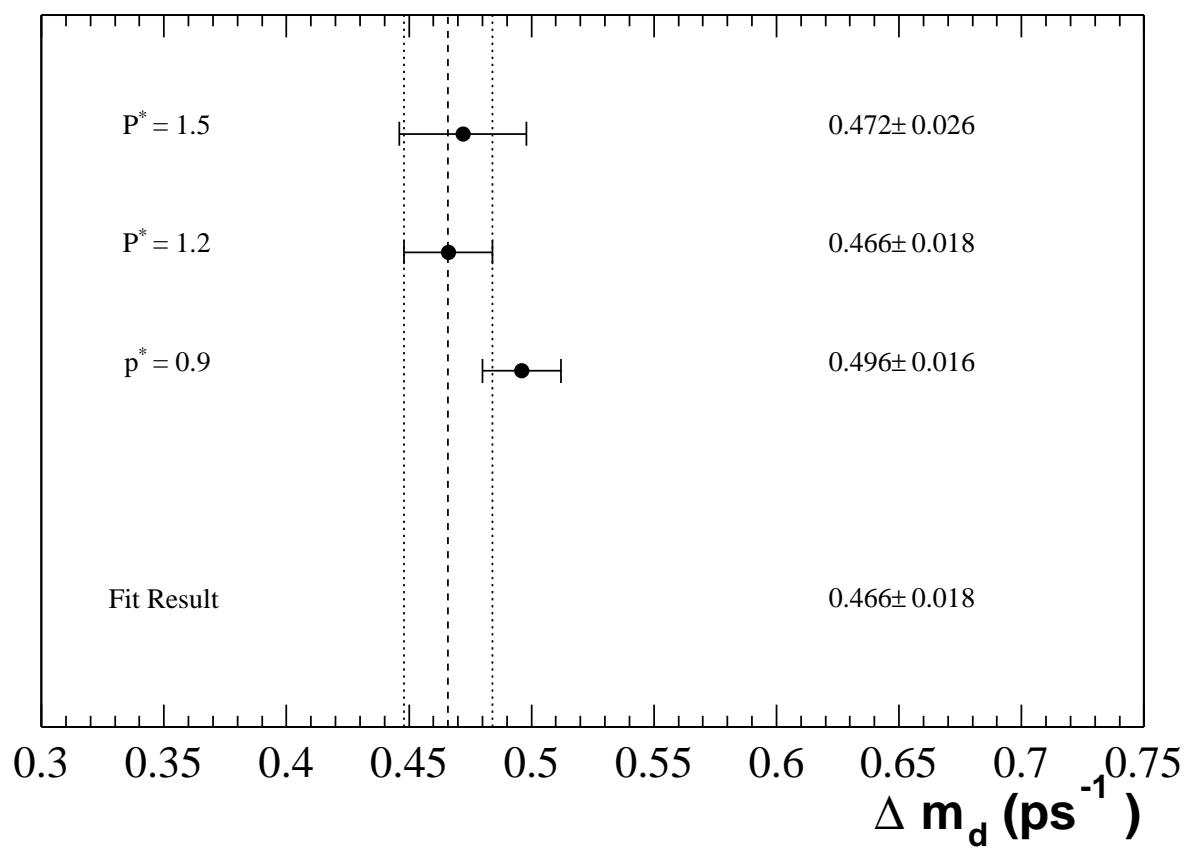

Figure 5.21: $\Delta m_{d}$ results for different lower bounds on the $p^{*}$ cut. The cut used in the analysis of $1.2 \mathrm{GeV} / \mathrm{c}$ is within the stability region of $\Delta m_{d}$. 


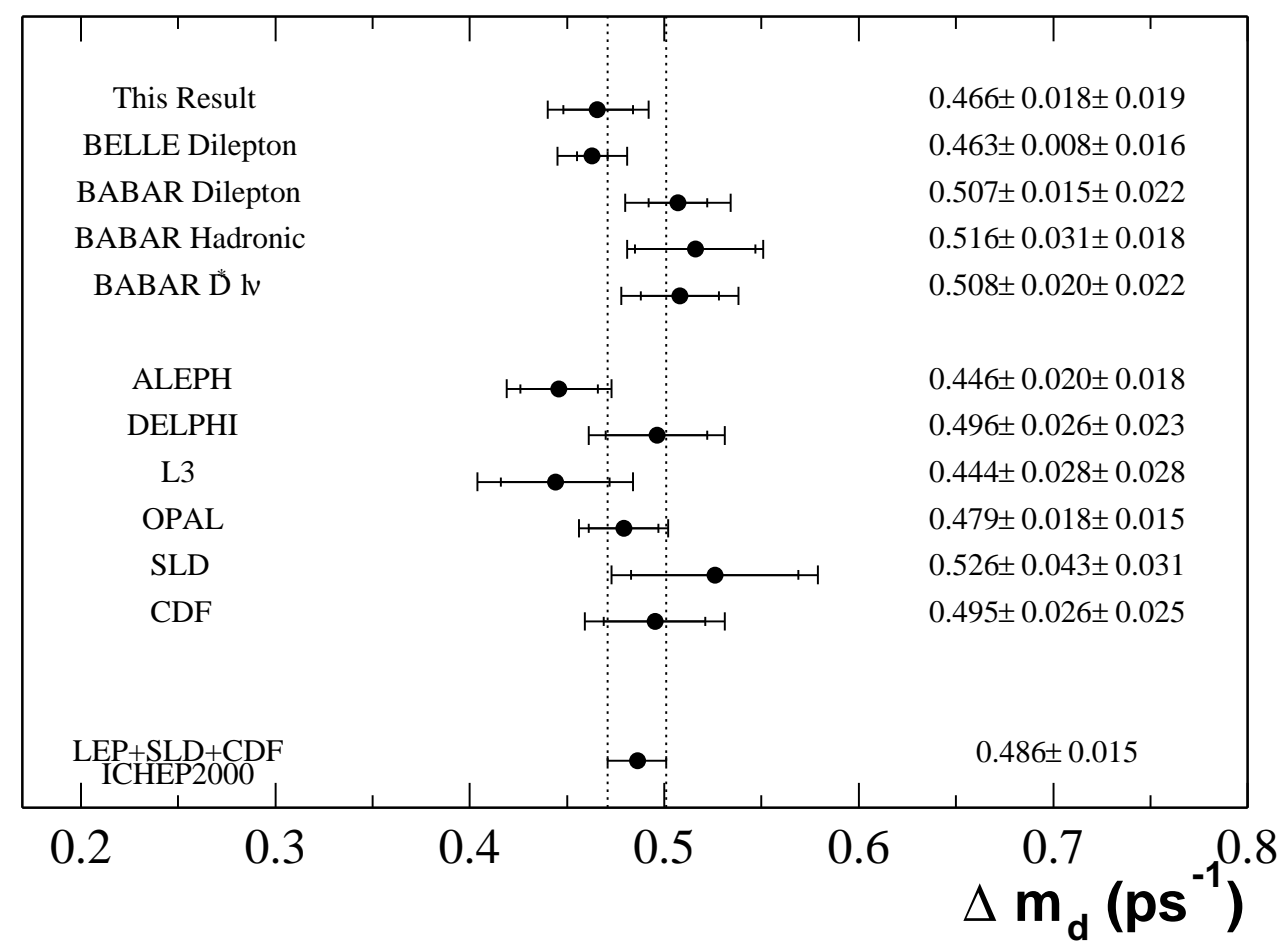

Figure 5.22: A summary of the $\Delta m_{d}$ results to date. The average is calculated using the LEP, SLD and CDF ICHEP 2000 results and does not include the fit result or the BABAR or BELLE results. 


\section{Chapter 6}

\section{Conclusions}

A full test of the SM involves the validation of its predictions and parameters to a very high precision. Any experimental deviation of a SM prediction or parameter from the theoretical value is an indication of new physics. The BABAR experiment built at the PEP-II asymmetric collider has been designed to do a systematic study of $\mathrm{CP}$ violation and precision measurements within the B meson system. The detector was commissioned in the summer of 1999 and recorded its first event on the $26^{\text {th }}$ of May 1999.

To fully exploit the physics potential at PEP-II it was imperative that BABAR utilized an effective and efficient DAQ system. The BABAR DAQ system has been designed operate at a Level 1 rate of $2 \mathrm{kHz}$. A description of the BABAR DAQ system and the functionality specific to the EMC was given in chapter 3. As the EMC front end electronics transmit a continuous stream of data, ROMs with the untriggered version of the personality cards are used by the EMC for data taking.

The simulation software used by BABAR has been designed to give an accurate description of the detector. In doing this, the inclusion of machine background noise is an important consideration. Two different methods, GHit mixing and event mixing, used for this purpose were presented. The GHit mixer uses simulated backgrounds whilst the event mixer has the functionality to use both real and simulated backgrounds. Following a discussion of the implementation of GHit mixing within the calorimeter simulation, the software written to facilitate event mixing was presented. A comparison of the two methods using MC backgrounds was given and the 
results were found to be in agreement. Due to the high energy resolution required, the EMC employs a matched digital filter. It was possible to improve the obtained energy resolution by means of a polynomial fit. This fit resulted in an energy dependent improvement in the resolution of $4-40 \%$ and an improvement in the timing resolution of about $90 \%$ over the filtered case.

The very high semileptonic branching ratio of $\mathrm{B}$ mesons makes leptons ideal candidates to tag the flavour of the B. The sign of the lepton charge in this case is equal to the sign of the charge on the b quark in the B meson. This is used by the dilepton approach in the measurement of $\Delta m_{d}$. An overview of the selections procedure used to isolate dileptons was given in chapter 4. The BABAR electron and muon selection algorithms used were shown to have selection efficiencies of about $80 \%$ and $60 \%$ respectively in the momentum region of interest. It was concluded that leptons arising from the semileptonic decay of the charm provided the biggest source of backgrounds. These cascade leptons were seen to contribute in three different ways. Background contributions from other sources such as continuum, $J / \psi$, gamma conversions, Bhabha, radiative Bhabha and two photon events and the selection cuts used to reduce their contamination were then described. The selection procedure was seen to have an efficiency of about $18 \%$ with a purity of about $88 \%$ for signal MC.

In estimating the B decay vertices a POCA approach was used. The decay vertex was taken to be the POCA of the lepton track to the beamspot in the xy plane. It was shown that the $\Delta z$ between the two vertices was related to the proper time, $\Delta t$, by the boost approximation. The resolution in z was calculated using two different methods; a global resolution function and an event-by-event error resolution function. It was concluded that most significant residual backgrounds could be parameterised in terms of resolution smeared exponential terms. In doing this, the contributions to the mixed and unmixed distributions were treated separately. It was shown that the convolution of the unmixed and mixed pdfs with the resolution function could be solved analytically. A simultaneous unbinned maximum likelihood fit to the unmixed and mixed distributions was used in the extraction of $\Delta m_{d}$. The fit procedure was validated using MC where the fit was seen to reproduce the simulated value. From the fit to the $7.72 \mathrm{fb}^{-1}$ of on-resonance data, $\Delta m_{d}$ was found 
to be $0.466 \pm 0.018 \mathrm{~h} \mathrm{ps}^{-1}$ with the mistag fraction, $\eta=0.211 \pm 0.008$ (the errors in this case are purely statistical). A summary of the systematic effects affecting the measurement was presented. The total systematic error was calculated to be $0.019 \mathrm{~h} \mathrm{ps}^{-1}$. The final result including the systematic errors was found to be $\Delta m_{d}$ $=0.466 \pm 0.018 \pm 0.019 \mathrm{~h} \mathrm{ps}^{-1}$. 


\section{Appendix A}

\section{Second order polynomial fit to an EmcWaveform}

Consider a waveform with an energy $y$ corresponding to bin $x$. Let the maximum of the waveform and the corresponding time bin be given by $y_{2}$ and $x_{2}$ respectively. Let the energy and bin number of the two adjacent bins be $\left(y_{1}, x_{1}\right)$ and $\left(y_{3}, x_{3}\right)$. Consider now a polynomial fit given by,

$$
y=a x^{2}+b x+c
$$

to the three points $\left(x_{1}, y_{1}\right),\left(x_{2}, y_{2}\right)$ and $\left(x_{3}, y_{3}\right)$. Since they are adjacent bins,

$$
\begin{aligned}
& x_{2}-x_{1}=1 \\
& x_{3}-x_{2}=1
\end{aligned}
$$

The parameters $a, b$ and $c$, are then given by,

$$
\begin{aligned}
a & =\frac{y_{1}+y_{3}-2 y_{2}}{2} \\
b & =\frac{\left(\left(y_{2}-y_{1}\right)\left(x_{3}+x_{2}\right)-\left(y_{3}-y_{2}\right)\left(x_{1}+x_{2}\right)\right)}{2} \\
c & =y_{1}-a x_{1}^{2}-b x_{1}
\end{aligned}
$$

The energy or the maximum value of $y, y_{\max }$, is given by, 


$$
y_{\max }=c-\frac{b^{2}}{4 a}
$$

and the time corresponding to this energy, $x_{\max }$, is given by,

$$
x_{\max }=-\frac{b}{2 a}
$$




\section{Appendix B}

\section{Analytical form of the convoluted lifetime pdf}

The lifetime pdf used in the maximum likelihood fit to the lifetime time distribution consisted of an exponential term that was convoluted with a Gaussian resolution function. The convolution integral can be solved analytically and the following is a derivation of this analytical form. The derivation is done for a convolution with a single Gaussian with no bias and a fixed sigma. The extension to the case of a bias and/or event-by-event errors is then trivial.

The starting point in this exercise is a normalised lifetime pdf given by,

$$
\mathcal{P}_{\text {life }}(t)=\frac{1}{\tau} e^{-t / \tau}
$$

and a normalised Gaussian resolution function given by,

$$
\mathcal{G}_{\text {res }}(t)=\frac{1}{\sqrt{2 \pi} \sigma} e^{-t^{2} / 2 \sigma^{2}}
$$

The convolution of B.1 with B.2 is then given by,

$$
\mathcal{P}_{\text {fit }}\left(t^{\prime}\right)=N \int_{0}^{\infty} e^{-t / \tau} e^{-\left(t-t^{\prime}\right)^{2} / 2 \sigma^{2}} d t
$$

where, the normalisation factor, $\mathrm{N}$, is given by

$$
N=\frac{1}{\sqrt{2 \pi} \sigma \tau}
$$

Expanding the expression in B.3 and forming a perfect square, one obtains, 


$$
\mathcal{P}_{f i t}\left(t^{\prime}\right)=N e^{\left(\frac{\sigma^{2}}{2 \tau^{2}}-\frac{t^{\prime}}{\tau}\right)} \int_{0}^{\infty} e^{-\frac{1}{2 \sigma^{2}}\left\{t+\left(\frac{\sigma^{2}}{\tau}-t^{\prime}\right)\right\}^{2}} d t
$$

The above can then be integrated by substitution. In doing this let,

$$
u=\frac{t}{\sqrt{2} \sigma}-\frac{1}{\sqrt{2} \sigma}\left(\frac{\sigma^{2}}{\tau}-t^{\prime}\right)
$$

so,

$$
\frac{d u}{d t}=\frac{1}{\sqrt{2} \sigma}
$$

Substituting the above into B.5,

$$
\mathcal{P}_{f i t}\left(t^{\prime}\right)=N \sqrt{2} \sigma e^{\left(\frac{\sigma^{2}}{2 \tau^{2}}-\frac{t^{\prime}}{\tau}\right)} \int_{x}^{\infty} e^{-u^{2}} d u
$$

here, $x$ is given by

$$
x\left(t^{\prime}\right)=\frac{1}{\sqrt{2} \sigma}\left(\frac{\sigma^{2}}{\tau}-t^{\prime}\right)
$$

The complementary error function $\operatorname{erfc}(\mathrm{x})$ is defined as,

$$
\operatorname{erfc}(x)=\frac{2}{\sqrt{\pi}} \int_{x}^{\infty} e^{-u^{2}} d u
$$

Substituting the definition of $N$ from (B.4) the convoluted lifetime pdf can be expressed as,

$$
\mathcal{P}_{f i t}\left(t^{\prime}\right)=\frac{1}{2 \tau} e^{\left(\frac{\sigma^{2}}{2 \tau^{2}}-\frac{t^{\prime}}{\tau}\right)} \operatorname{erfc}(x)
$$

where $\mathrm{x}$ is given by (B.7). The above expression can be extended for the case where event-by-event errors are used. If the error scaling factor associated with $t^{\prime}$ is given by $\epsilon$, the two dimensional pdf is then given by,

$$
\mathcal{P}_{f i t}\left(t^{\prime}, \epsilon\right)=\frac{1}{2 \tau} e^{\left(\frac{\sigma^{2} \epsilon^{2}}{2 \tau^{2}}-\frac{t^{\prime}}{\tau}\right)} \operatorname{erfc}(y)
$$

where $y$, is given by,

$$
y\left(t^{\prime}, \epsilon\right)=\frac{1}{\sqrt{2} \sigma \epsilon}\left(\frac{\sigma^{2} \epsilon^{2}}{\tau}-t^{\prime}\right)
$$




\section{Appendix C}

\section{Analytical forms of the convoluted unmixed and mixed pdfs}

The derivation of the analytical form of the unmixed and mixed pdfs will be given in this section. The pure (non-background mixed) unmixed and mixed pdfs consist of a lifetime term and an oscillatory term. The derivation of the convolution of the lifetime term with the resolution function was given in Appendix B. Here, the analytical form of the convolution of the oscillatory term with the resolution function will be derived. Once again, for clarity and simplicity, the convolution will be done for a single Gaussian global resolution function. The result thus obtained will then be extended to the case where event-by-event errors are used. As it is possible to express the backgrounds as convoluted lifetime distributions, this derivation will not include any such backgrounds.

The unmixed and mixed pdfs are given by,

$$
\begin{aligned}
\mathcal{P}_{\text {unmixed }}(t) & \propto e^{-t / \tau}\left[1+D \cos \left(\Delta m_{d} t\right)\right] \\
\mathcal{P}_{\text {mixed }}(t) & \propto e^{-t / \tau}\left[1-D \cos \left(\Delta m_{d} t\right)\right]
\end{aligned}
$$

where, the dilution factor, $\mathrm{D}$, can be expressed in terms of the the mistag rate $(\eta)$ as,

$$
D=1-2 \eta
$$


Consider now the convolution of the unmixed pdf with a normalised Gaussian distribution given by,

$$
\mathcal{G}_{\text {res }}(t)=\frac{1}{\sqrt{2 \pi} \sigma} e^{-t^{2} / 2 \sigma^{2}}
$$

The convolution integral is given by,

$$
\mathcal{P}_{\text {unmixed }}\left(t^{\prime}\right)=\mathcal{N}_{\text {unmixed }} \int_{0}^{\infty} e^{-t / \tau}\left[1+D \cos \left(\Delta m_{d} t\right)\right] e^{-\left(t-t^{\prime}\right)^{2} / 2 \sigma^{2}} d t
$$

with a similar expression for the mixed distribution.

$$
\mathcal{P}_{\text {mixed }}\left(t^{\prime}\right)=\mathcal{N}_{\text {mixed }} \int_{0}^{\infty} e^{-t / \tau}\left[1-D \cos \left(\Delta m_{d} t\right)\right] e^{-\left(t-t^{\prime}\right)^{2} / 2 \sigma^{2}} d t
$$

The normalisation factors, $\mathcal{N}_{\text {unmixed }}$ and $\mathcal{N}_{\text {mixed }}$ will be calculated later. The derivation will now be done for the unmixed distribution. The expression in equation C.4 can be expanded as,

$$
\begin{array}{r}
\mathcal{P}_{\text {unmixed }}\left(t^{\prime}\right)=N_{\text {unmixed }}\left(\int_{0}^{\infty} e^{-t / \tau} e^{-\left(t-t^{\prime}\right)^{2} / 2 \sigma^{2}} d t\right. \\
\left.+\int_{0}^{\infty} e^{-t / \tau} D \cos \left(\Delta m_{d} t\right) e^{-\left(t-t^{\prime}\right)^{2} / 2 \sigma^{2}} d t\right)
\end{array}
$$

The first term in equation C.6 is just a convolution of a lifetime type term and using the derivation in Appendix B,

$$
\int_{0}^{\infty} e^{-t / \tau} e^{-\left(t-t^{\prime}\right)^{2} / 2 \sigma^{2}} d t \propto e^{\left(\frac{\sigma^{2}}{2 \tau^{2}}-\frac{t^{\prime}}{\tau}\right)} \operatorname{erfc}(x)
$$

where, $\mathrm{x}$ is given by,

$$
x\left(t^{\prime}\right)=\frac{1}{\sqrt{2} \sigma}\left(\frac{\sigma^{2}}{\tau}-t^{\prime}\right)
$$

and $\operatorname{erfc}(\mathrm{x})$ is the complementary error function defined in equation B.8. Consider now the integral involving the cosine term, 


$$
\mathcal{I}\left(t^{\prime}\right)=N_{\text {unmixed }} \int_{0}^{\infty} e^{-t / \tau} D \cos \left(\Delta m_{d} t\right) e^{-\left(t-t^{\prime}\right)^{2} / 2 \sigma^{2}} d t
$$

This cosine term can be expressed in terms of complex exponentials as,

$$
\cos \left(\Delta m_{d} t\right)=\frac{e^{i \Delta m_{d} t}+e^{-i \Delta m_{d} t}}{2}
$$

The convolution integral then becomes,

$$
\begin{gathered}
\mathcal{I}\left(t^{\prime}\right)=N_{\text {unmixed }} \int_{0}^{\infty} e^{-t / \tau} D\left(\frac{e^{i \Delta m_{d} t}+e^{-i \Delta m_{d} t}}{2}\right) e^{-\left(t-t^{\prime}\right)^{2} / 2 \sigma^{2}} d t \\
\mathcal{I}\left(t^{\prime}\right)=N_{\text {unmixed }} \frac{D}{2}\left(\int_{0}^{\infty} e^{-t / \tau}\left(e^{i \Delta m_{d} t}\right) e^{-\left(t-t^{\prime}\right)^{2} / 2 \sigma^{2}} d t\right. \\
\left.+\int_{0}^{\infty} e^{-t / \tau}\left(e^{-i \Delta m_{d} t}\right) e^{-\left(t-t^{\prime}\right)^{2} / 2 \sigma^{2}} d t\right) \\
\mathcal{I}\left(t^{\prime}\right)=N_{\text {unmixed }} \frac{D}{2}\left(\mathcal{I}_{+}\left(t^{\prime}\right)+\mathcal{I}_{-}\left(t^{\prime}\right)\right)
\end{gathered}
$$

where, $\mathcal{I}_{+}\left(t^{\prime}\right)$ and $\mathcal{I}_{-}\left(t^{\prime}\right)$ are given by,

$$
\begin{gathered}
\mathcal{I}_{+}\left(t^{\prime}\right)=\int_{0}^{\infty} e^{-t / \tau}\left(e^{i \Delta m_{d} t}\right) e^{-\left(t-t^{\prime}\right)^{2} / 2 \sigma^{2}} d t \\
\mathcal{I}_{-}\left(t^{\prime}\right)=\int_{0}^{\infty} e^{-t / \tau}\left(e^{-i \Delta m_{d} t}\right) e^{-\left(t-t^{\prime}\right)^{2} / 2 \sigma^{2}} d t
\end{gathered}
$$

For simplicity consider only the $\mathcal{I}_{+}\left(t^{\prime}\right)$ term. Expanding the term in the exponent, and forming a perfect square, the above can be written as,

$$
\mathcal{I}_{+}\left(t^{\prime}\right)=e^{-t^{\prime 2} / 2 \sigma^{2}} \int_{0}^{\infty} e^{-1 / 2 \sigma^{2}\left(t^{2}+2 t k\right)} d t
$$

where, $\mathrm{k}$ is given by,

$$
k=\frac{\sigma^{2}}{\tau}-t^{\prime}-i \sigma^{2} \Delta m_{d}
$$


the above can then be integrating by substitution. Defining the substitution variable, $\mathrm{u}$ as,

$$
u=\frac{t+k}{\sqrt{2} \sigma}
$$

the above integral can be written as,

$$
\mathcal{I}_{+}\left(t^{\prime}\right)=e^{-t^{\prime 2} / 2 \sigma^{2}} e^{x^{2}} \sqrt{2} \sigma \int_{x}^{\infty} e^{-u^{2}} d u
$$

here, the complex lower bound on the integral, $x$, is given by

$$
x=\frac{k}{\sqrt{2} \sigma}=\frac{\sigma}{\sqrt{2} \tau}-\frac{t^{\prime}}{\sqrt{2} \sigma}-i \frac{\sigma \Delta m_{d}}{\sqrt{2}}
$$

Equation C.12 can be written in terms of the complementary error function defined in equation B.8 as,

$$
\mathcal{I}_{+}\left(t^{\prime}\right)=\sigma \sqrt{\frac{\pi}{2}} e^{-t^{\prime 2} / 2 \sigma^{2}} e^{x^{2}} \operatorname{erfc}(x)
$$

The complex error function, $w(z)$ is defined as,

$$
w(z)=e^{-z^{2}} \operatorname{erfc}(-i z)
$$

defining $z$ to be $z=i x$,

$$
z=i x=\frac{\sigma \Delta m_{d}}{\sqrt{2}}+i\left(\frac{\sigma}{\sqrt{2} \tau}-\frac{t^{\prime}}{\sqrt{2} \sigma}\right)
$$

thus,

$$
e^{x^{2}} \operatorname{erfc}(x)=e^{-z^{2}} \operatorname{erfc}(-i z)=w(z)
$$

$\mathcal{I}_{+}\left(t^{\prime}\right)$ can be thus expressed as,

$$
\mathcal{I}_{+}\left(t^{\prime}\right)=\sigma \sqrt{\frac{\pi}{2}} e^{-t^{\prime 2} / 2 \sigma^{2}} w(z)
$$

Similarly, it can be shown that $\mathcal{I}_{-}\left(t^{\prime}\right)$ is given by, 


$$
\mathcal{I}_{-}\left(t^{\prime}\right)=\sigma \sqrt{\frac{\pi}{2}} e^{-t^{\prime 2} / 2 \sigma^{2}} w\left(-z^{*}\right)
$$

Thus, substituting equations C.18 and C.17 into equation C.11 one gets,

$$
\mathcal{I}\left(t^{\prime}\right)=\frac{D}{2} e^{-t^{\prime 2} / 2 \sigma^{2}} \sqrt{\frac{\pi}{2}} \sigma\left(w(z)+w\left(-z^{*}\right)\right)
$$

The normalisation factors, $N_{\text {unmixed }}$ and $N_{\text {mixed }}$ will now be calculated. Consider first the unmixed case. This is subject to,

$$
1=N_{\text {unmixed }} \frac{1}{\sqrt{2 \pi} \sigma} \int_{0}^{\infty} e^{-t / \tau}\left[1+D \cos \left(\Delta m_{d} t\right)\right] d t
$$

with the factor of $\frac{1}{\sqrt{2 \pi} \sigma}$ coming from the normalisation of the Gaussian resolution function. The above can be expanded and solved by using integration by parts twice to yield,

$$
N_{\text {unmixed }}=\frac{1}{\sqrt{2 \pi} \sigma}\left(\tau+\frac{D \tau}{1+\tau^{2} \Delta m_{d}^{2}}\right)^{-1}
$$

similarly, it can be shown that,

$$
N_{\text {mixed }}=\frac{1}{\sqrt{2 \pi} \sigma}\left(\tau-\frac{D \tau}{1+\tau^{2} \Delta m_{d}^{2}}\right)^{-1}
$$

Substituting C.19, B.9 and C.21 into C.6,

$$
\begin{aligned}
& \mathcal{P}_{\text {unmixed }}(t)=\frac{1}{\sqrt{2 \pi} \sigma}\left(\tau+\frac{D \tau}{1+\tau^{2} \Delta m_{d}^{2}}\right)^{-1} \\
& \sqrt{\frac{\pi}{2}} \sigma\left[e^{\left(\frac{\sigma^{2}}{2 \tau^{2}}-\frac{t^{\prime}}{\tau}\right)} \operatorname{erfc}(\mathrm{x})+\frac{\mathrm{D}}{2} \mathrm{e}^{-\mathrm{t}^{\prime 2} / 2 \sigma^{2}}\left(\mathrm{w}(\mathrm{z})+\mathrm{w}\left(-\mathrm{z}^{*}\right)\right)\right]
\end{aligned}
$$

after some cancellations, this yields,

$$
\mathcal{P}_{\text {unmixed }}(t)=\mathcal{N}_{\text {unmixed }}\left[e^{\left(\frac{\sigma^{2}}{2 \tau^{2}}-\frac{t^{\prime}}{\tau}\right)} \operatorname{erfc}(\mathrm{x})+\frac{\mathrm{D}}{2} \mathrm{e}^{-\mathrm{t}^{\prime 2} / 2 \sigma^{2}}\left(\mathrm{w}(\mathrm{z})+\mathrm{w}\left(-\mathrm{z}^{*}\right)\right)\right]
$$

where, $\mathcal{N}_{\text {unmixed }}$ is given by,

$$
\mathcal{N}_{\text {unmixed }}=\frac{1}{2}\left(\tau+\frac{D \tau}{1+\tau^{2} \Delta m_{d}^{2}}\right)^{-1}
$$


and $\mathrm{x}$ is given by equation C.8 and $\mathrm{z}$ is given by,

$$
z\left(t^{\prime}\right)=\frac{\sigma \Delta m_{d}}{\sqrt{2}}+i\left(\frac{\sigma}{\sqrt{2} \tau}-\frac{t^{\prime}}{\sqrt{2} \sigma}\right)
$$

Similarly, the mixed pdf can be written as,

$$
\mathcal{P}_{\text {mixed }}(t)=\mathcal{N}_{\text {mixed }}\left[e^{\left(\frac{\sigma^{2}}{2 \tau^{2}}-\frac{t^{\prime}}{\tau}\right)} \operatorname{erfc}(\mathrm{x})-\frac{\mathrm{D}}{2} \mathrm{e}^{-\mathrm{t}^{\prime 2} / 2 \sigma^{2}}\left(\mathrm{w}(\mathrm{z})+\mathrm{w}\left(-\mathrm{z}^{*}\right)\right)\right]
$$

with $\mathcal{N}_{\text {mixed }}$ given by,

$$
\mathcal{N}_{\text {mixed }}=\frac{1}{2}\left(\tau-\frac{D \tau}{1+\tau^{2} \Delta m_{d}^{2}}\right)^{-1}
$$

This can be then expanded for the case where event-by-event errors are used. If the error scaling factor associated with event $t^{\prime}$ is given by $\epsilon$, the unmixed and mixed pdfs given by

$$
\mathcal{P}_{\text {unmixed }}\left(t^{\prime}, \epsilon\right)=\mathcal{N}_{\text {unmixed }}\left[e^{\left(\frac{\sigma^{2} \epsilon^{2}}{2 \tau^{2}}-\frac{t^{\prime}}{\tau}\right)} \operatorname{erfc}(\mathrm{y})+\frac{\mathrm{D}}{2} \mathrm{e}^{-\mathrm{t}^{\prime} / 2 \sigma^{2} \epsilon^{2}}\left(\mathrm{w}(\mathrm{m})+\mathrm{w}\left(-\mathrm{m}^{*}\right)\right)\right]
$$

and

$$
\mathcal{P}_{\text {mixed }}\left(t^{\prime}, \epsilon\right)=\mathcal{N}_{\text {mixed }}\left[e^{\left(\frac{\sigma^{2} \epsilon^{2}}{2 \tau^{2}}-\frac{t^{\prime}}{\tau}\right)} \operatorname{erfc}(\mathrm{y})-\frac{\mathrm{D}}{2} \mathrm{e}^{-\mathrm{t}^{\prime 2} / 2 \sigma^{2} \epsilon^{2}}\left(\mathrm{w}(\mathrm{m})+\mathrm{w}\left(-\mathrm{m}^{*}\right)\right)\right]
$$

here, $\mathrm{y}$ and $\mathrm{m}$ are given by

$$
y\left(t^{\prime}, \epsilon\right)=\frac{1}{\sqrt{2} \sigma \epsilon}\left(\frac{\sigma^{2} \epsilon^{2}}{\tau}-t^{\prime}\right)
$$

and

$$
m\left(t^{\prime}, \epsilon\right)=\frac{\sigma \epsilon \Delta m_{d}}{\sqrt{2}}+i\left(\frac{\sigma \epsilon}{\sqrt{2} \tau}-\frac{t^{\prime}}{\sqrt{2} \sigma \epsilon}\right)
$$




\section{Bibliography}

[1] J. H. Christenson, Phys. Rev. Lett. 13, (1964) 138.

[2] A. D. Sakharov, ZhETF Pis. Red. 5 (1967) 32; JETP Lett. 5 (1967) 24.

[3] G. R. Farrar \& M. E. Shaposhnikov, Phys. Rev. D 50 (1994) 744.

[4] M. B. Gavela et al., Nucl. Phys. B430 (1994) 382.

[5] P. Huet \& E. Sather, Phys. Rev. D 51 (1995) 379.

[6] A. G. Cohen, D. B. Kaplan \& A. E. Nelson, Ann. Rev. Nucl. Part. Sci. 43 (1993) 27.

[7] Y. Nir, "CP Violation in and Beyond the Standard Model", SLAC Summer Institute, (1999).

[8] R. N. Mohapatra, "Unification and Supersymmetry", Springer (1986).

[9] R. E. Marshak, "Conceptual Foundations of Modern Particle Physics", World Scientific (1993) 367-376.

[10] C. Jarlskog, Phys. Rev. Lett. 55 (1985) 1039.

[11] P. H. Frampton, "Gauge Field Theories", Frontiers in Physics (Benjamin/Cummings Publishing) (1987).

[12] M. Kobayashi \& T. Maskawa, Prog. Th. Phys. 49 (1973) 652.

[13] H.Harari \& M. Leurer, Phys. Lett. B 181 (1986) 123.

[14] L. Wolfenstein, Phys. Rev. Lett. 51 (1983) 1945. 
[15] A. J. Buras \& R. Fleischer, hep-ph/9704376.

[16] S. Weinberg, Phys. Rev. Lett. 19 (1967) 1264.

[17] E. Leader \& E. Predazzi, "An Introduction to Gauge Theories and Modern Particle Physics", Vol 2 (Cambridge University Press) (1996) p.12.

[18] R. E. Marshak, Riazuddin \& C. P. Ryan, "The Theory of Weak Interactions in Particle Physics" (Wiley, New York) (1969).

[19] O. Nachtman, "Elementary Particle Physics", Springer-Verlag (1990).

[20] G. D. Barr et al., (NA31 Collaboration) Phys. Lett. B 317 (1993) 233.

[21] L. K. Gibbons et al., (E731 Collaboration) Phys. Rev. Lett. 70 (1993) 1203.

[22] A. Buras, hep-ph/9901409 (1999).

[23] G. Buchalla, A. Buras \& M. Lautenbacher, Rev. Mod. Phys. 68 (1996) 1125.

[24] K. G. Wilson, Phys. Rev. 179 (1969) 1499.

[25] The BaBar Physics Book (ed. P.F Harrison \& H. R. Quinn) SLAC-R-504 (1998)

[26] Particle Data Group, Eur. Phys. Jour. C 15 (2000) 1.

[27] V. Sharma, http://www.slac.stanford.edu/BFROOT/www/Physics/ official/sin2beta/dmd_world.eps

[28] The BABAR Technical Design Report, SLAC-R-95-457 (1995).

[29] The PEP-11 Conceptual Design Report, SLAC-PUB-418 (1993).

[30] B. Aubert et al., (BABAR collaboration), "The first year of the BABAR experiment at PEP-II", Paper submitted to the $30^{\text {th }}$ International Conference on High Energy Physics, Osaka Japan (2000).

[31] A. Snyder, "Effects of vertex cuts on CP reach", BABAR Note \#177 (1994).

[32] D. Barbieri et al., "Silicon Sensors for the BABAR Vertex Tracker", submitted to Nuovo Cimento. 
[33] F.Lanni \& F. Palombo, Nucl. Instr. Methods A379 (1996) 399.

[34] G. Sciolla, "The BABAR Drift Chamber", Proceedings of the 8th Vienna Wire Chamber Conference, SLAC-PUB-7779 (1998).

[35] R. Johnson et al., "Silicon Vertex Detector Readout Chip, Requirement Specification", BABAR note \#213 (1995).

[36] R. Johnson et al., "Silicon Vertex Detector Readout Chip (Target Design Specification)", BABAR note \#214 (1995).

[37] A. Chau et al., IEEE Trans. Nucl. Sci. 43 (1996) 1720.

[38] The trigger group, http://www.slac.stanford.edu/BFROOT/www/Detector/Trigger/ operations/trigdef.html.

[39] E Frank et al., "Architecture of the BABAR L3 software trigger", Proceedings of CHEP 98 (1998)

[40] R.T Hamilton et al., "The DataFlow Platform User's Guide”, BABAR Note \#385 (1998).

[41] VITA, "The VMEbus Specification”, ANSI/IEEE STD 1014-1987, ICE 821 and 297.

[42] L. Sapozhnikov, "Architecture for the BABAR Fast Control Timing System" http://www.slac.stanford.edu/ leosap/fcts.html.

[43] G. Haller, "The Conceptual Design of the BABAR Fast Control Timing System" http://www.slac.stanford.edu/BFROOT/www/Detector/DAQ/ Fast_Cntrl_Tim_System/index.html.

[44] G. Haller \& D. R. Freytag, "Analogue Floating-Point BiCMOS Sampling Chip and Architecture of the BABAR CSI Calorimeter Front-End Electronics System at the SLAC B-Factory", BABAR Note \#285 (1995). 
[45] J. Nash, P. M. Sanders \& P.D. Strother, "Investigation of the Matched Digital Filter Algorithms", BABAR Note \#274 (1996).

[46] P. D. Strother, "Investigation \& Simulation of the BABAR Calorimeter", 1st year report, Imperial College (1996).

[47] J.J Olsen et al., "BABAR DAQ ROM Untriggered Personality Card Description" http://www.slac.stanford.edu/BFROOT/www/Detector/DAQ/

ReadoutModule/rom.html.

[48] G. Booch, "Object Oriented Analysis and Design", (2nd ed.), (Addison-Wesley, California) (1994).

[49] A. Ryd et al., "EvtGev User Guide" http://www.slac.stanford.edu:/BFROOT/doc/www/

Physics/generators/EvtGen/EvtGen.html.

[50] T. Sjostrand, "Pythia 5.7 and Jetset 7.4 Physics and Manual", CERN-TH $7112 / 93$ (1993).

[51] D. Wright, "Beget : The B-Factory Event Generator Version 21", BABAR Note \#149 (1994).

[52] GEANT Detector Description and Simulation Tool, Version 3.15, CERN Program Library W5103 (CERN, Geneva) (1993).

[53] K. L. Brown \& F. C. Iselin, Decay Turtle ("Trace Unlimited Rays Through Lumped Elements"),CERN 74-2, (CERN, Geneva) (1974).

[54] P. D. Strother, "Design and application of the reconstruction software for the BABAR Calorimeter", PhD thesis, Imperial College, (1999).

[55] E. Frank, http://www.slac.stanford.edu/BFROOT/dist/releases/8.4.1/trgFrame/README.

[56] N. J. W. Gunawardane, "Investigation of the BABAR EMC Digital Filter Algorithms", BABAR Note \#487 (1998). 
[57] H. Albrecht et al., (ARGUS Collaboration), Phys. Lett. B 374 (1996) 256.

[58] J. Bartelt et al., (CLEO Collaboration), Phys. Rev. Lett. 71 (1993) 1680.

[59] A. Drescher et al., Nucl. Instr. Methods A237 (1985) 464.

[60] R. Sinkus \& T. Voss, Nucl. Instr. Methods A391 (1997) 360.

[61] Electron Identification Analysis Working Group, "Cut-Based Electron Identification", BABAR Analysis Document \#90, version 3 (2000).

[62] Muon Identification Analysis Working Group, "Muon Identification in the BABAR experiment", BABAR Analysis Document \#60, version 1 (2000).

[63] B. Aubert et al., (BABAR Collaboration), "Measurement of the time dependence of $B^{0} \bar{B}^{0}$ oscillations using inclusive dilepton events", Paper submitted to the $30^{\text {th }}$ International Conference on High Energy Physics, Osaka Japan (2000).

[64] C. Cheng et al., "Beamspot determination and use in BABAR", BABAR Analysis Document \#13, version 4 (2000).

[65] The Belle Collaboration, "Measurement of $B_{d}^{0}-\bar{B}_{d}^{0}$ mixing rate from the time evolution of dilepton events at the $\Upsilon(4 \mathrm{~S})$ ", submitted to Phys. Rev. Lett. , hep-ex/0011090v2 (2000). 